\title{
Un arc monumental sévérien à Bayeux (Calvados)
}

A monumental arch from the Severan period in Bayeux (Calvados)

Un arco monumental severiano en Bayeux (Calvados)

Ein monumentaler Bogen aus der severerische Zeit in Bayeux (Calvados,

Frankreich)

Yvan Maligorne, Grégory Schütz et Annabelle Cocollos (Illustration de)

\section{(2) OpenEdition} Journals

Édition électronique

URL : http://journals.openedition.org/rao/3088

DOI : $10.4000 /$ rao.3088

ISBN : 978-2-7535-5014-8

ISSN : 1775-3732

\section{Éditeur}

Presses universitaires de Rennes

\section{Édition imprimée}

Date de publication : 31 décembre 2015

Pagination : 317-362

ISBN : 978-2-7535-5012-4

ISSN : 0767-709X

\section{Référence électronique}

Yvan Maligorne, Grégory Schütz et Annabelle Cocollos (Illustration de), « Un arc monumental sévérien à Bayeux (Calvados) », Revue archéologique de l'Ouest [En ligne], 32 | 2015, mis en ligne le 28 avril 2018, consulté le 02 mars 2021. URL : http://journals.openedition.org/rao/3088 ; DOI : https://doi.org/ $10.4000 /$ rao.3088 


\title{
Un arc monumental sévérien à Bayeux (Calvados)
}

\author{
A Monumental Arch from the Severan Period in Bayeux (Calvados)
}

\author{
Yvan Maligorne et Grégory SchüTz ${ }^{\mathrm{b}}$, \\ avec des illustrations d'Annabelle Cocollos ${ }^{c}$
}

\begin{abstract}
Résumé : Vingt blocs d'architecture découverts à Bayeux au XIX ${ }^{\mathrm{e}}$ siècle constituent les seuls vestiges connus d'un arc monumental datant de l'époque sévérienne. Son architecture peut être restituée dans ses grandes lignes : le monument comportait trois baies, l'ouverture médiane étant sensiblement plus importante, et présentait une épaisseur réduite qui l'assimilait à une façade monumentale plus qu’à un volume autonome. Il était couvert d'un décor très abondant qui, à en juger par les bribes conservées, évoquait l'abondance et la felicitas auxquelles pourvoyait le pouvoir impérial.
\end{abstract}

\begin{abstract}
Twenty architectural blocks are the only known remains of a monumental arch dating from the Severan period. Its shape can be broadly restored: the monument had three bays, the central one being the widest, and its modest thickness made it look more like a monumental façade than a real autonomous volume. It was covered by a rich decoration, whose fragments are linked to two themes of the imperial ideology, the abundance and the felicitas.
\end{abstract}

Mots clés : arc à trois baies, période sévérienne, vigne, Dionysos.

Keywords: three-bay arch, Severan period, vine, Dionysos.

Alors que l'archéologie préventive s'efforce de profiter de la moindre occasion de fouilles pour dégager les vestiges antiques de tissus urbains souvent très denses, des documents essentiels sont encore trop fréquemment ignorés : les blocs d'architecture, susceptibles de fournir des informations irremplaçables sur la panoplie monumentale des villes, et d'abord sur les monuments publics et les tombeaux. Les études consacrées par Dominique Tardy (1989, 1994, 2005) aux collections de Saintes et de Périgueux en constituent l'illustration la plus éloquente : une mise en série systématique et des études comparatives bien conduites permettent de dégager des données sur la datation des monuments, sur la formation des artisans, sur le niveau des commanditaires et, dans les cas les plus favorables, sur la nature et la morphologie des programmes architecturaux.

Si elles n'ont ni l'ampleur ni la richesse de celles des grands centres urbains d'Aquitaine ou de Gaule Belgique, les collections lapidaires des villes de Lyonnaise occidentale ne sont pas pour autant dépourvues de valeur documentaire; on est en droit d'attendre de leur étude, qui reste à conduire dans les cités de l'actuelle Basse-Normandie, des progrès non négligeables dans la connaissance des organismes urbains : c'est ce que nous espérons illustrer dans cet article, qui déduit de l'étude de vingt blocs antiques mis au jour à Bayeux au milieu du XIX ${ }^{e}$ siècle l'existence d'un monument important, dont aucune trace n'est conservée par ailleurs.

\footnotetext{
${ }^{a}$ Centre de recherche bretonne et celtique (EA 4451), Faculté des lettres et sciences sociales, Université de Bretagne occidentale. (yvan.maligorne@yahoo.fr)

${ }^{\mathrm{b}}$ Archéologue, attaché de conservation du patrimoine, Service archéologie - Conseil général du Calvados, membre associé au Centre Michel de Boüard - CRAHAM - UMR 6273 (CNRS/UCBN). (gregory.schutz@calvados.fr)

c Graphiste, Service archéologie - Conseil général du Calvados.
} 
Ces blocs relèvent d'une typologie variée - pilastres, colonne engagée, claveaux - mais présentent une unité stylistique qui justifie leur rapprochement au sein d'une structure unique, en l'espèce un arc monumental comportant trois baies d'inégale ampleur.

Jamais soumis à une enquête systématique, ces blocs ont cependant fait l'objet de nombreuses mentions bibliographiques, lesquelles permettent d'en déterminer les circonstances et le contexte de découverte $(\$ 1)$. Après avoir dressé l'inventaire détaillé de ces éléments $(\$ 2)$, nous montrerons que le décor architectural non figuré permet non seulement d'avancer une datation assez précise - sévérienne -, mais encore d'identifier l'intervention d'un groupe de tailleurs de pierre dont l'activité était déjà documentée dans la région $(\$ 3)$. Les informations fournies par les blocs, alliées à une démarche comparative qui peut s'appuyer sur des séries monumentales particulièrement étoffées, conduisent à proposer une évocation - plus qu'une véritable restitution - de l'architecture du monument $(\$ 4)$. Les questions importantes et connexes liées à la valeur urbanistique de l'arc de Bayeux et à son contenu idéologique ne peuvent trouver de réponses assurées, mais les lambeaux de son décor figuré qui nous sont parvenus suggèrent quelques pistes de réflexion $(\$ 5)$. La conclusion nous offrira l'occasion de dresser un bilan - très rapide - sur le type monumental en Gaule occidentale.

\section{Contexte de découverte des blocs SCULPTÉS ET HISTORIQUE DES RECHERCHES AUTOUR DE LA CATHÉDRALE}

\section{Bayeux dans l'Antiquité}

Bayeux se situe dans le Calvados (Basse-Normandie) à $7 \mathrm{~km}$ au sud du littoral de la Manche et à $30 \mathrm{~km}$ au nordouest de Caen, la préfecture du département. La ville se développe principalement sur les bords de l'Aure, à l'emplacement d'Augustodurum, le chef-lieu de cité des Baïocasses (fig. 1). Ce peuple de Gaule Lyonnaise est mentionné pour la première fois par Pline l'Ancien dans la seconde moitié du I ${ }^{\text {er }}$ siècle de notre ère (Naturalis Historia, IV, 107 : Bodiocasses). La ville apparaît également sur la carte de Peutinger (Augustoduro). La découverte de trois nécropoles au nord-est, au sud-ouest et à l'ouest de l'agglomération permet d'estimer la superficie de l'espace urbain à près de 50 hectares (fig. 2). Son organisation est néanmoins mal connue, faute de rues attestées. Seul un modeste tronçon, orienté nord-ouest/sud-est, observé au cours des années 1860 dans une cave au ${ }^{\circ} 14$ de la rue Saint-Jean, vient étayer l'idée selon laquelle les rues Saint-Patrice, Saint-Malo et
Saint-Jean correspondraient à l'axe d'un decumanus (Lorillu 1891, p. 14; Dalibard 1977, p. 11; Quétier 1981, p. 10-15; Delacampagne, Paillard 1992, p. 13-14). Nous pouvons également citer une voie ponctuellement observée en 2011, correspondant à l'une des rues établies en périphérie de la ville (diagnostic au 13 rue de Nesmond : Schütz, Sauvin 2011a, p. 58-59, 64-66 et 92-93). L'agglomération, qui dispose par l'intermédiaire de l'Aure et de la Vire d'un accès indirect à la mer, s'insère dans un réseau de voies routières qui la met en relation avec les cités voisines - Viducasses et Lexoviens à l'est, Unelles à l'ouest et Abrincates au sud -, ainsi qu'avec le Bassin parisien, par l'intermédiaire notamment du "Chemin Haussé ", importante voie antique reliant la ville à Chartres. En dehors de deux ensembles thermaux fouillés au XIX ${ }^{\mathrm{e}}$ siècle ${ }^{1}$, de substructions observées sous la cathédrale (Delacampagne, 1990a; $n^{\circ} 43$ ) et d'une esplanade dallée à caractère public, observée en 2010 sur environ sept cents mètres carrés et interprétée comme l'aire libre du forum (Schütz, Sauvin 2011b; Schütz et al., 2013), les informations précises sur l'occupation gallo-romaine apparaissent dispersées. Les quelques mentions anciennes et plusieurs découvertes récentes attestent néanmoins l'existence d'ensembles de vestiges riches et bien conservés. L'agglomération correspondait à une ville de taille modeste.

Pendant l'Antiquité tardive, Augustodurum prend le nom de Civitas Baiocassium (Notitia Galliarum, II, 2) et devient une cité de la province de Lyonnaise Seconde; elle se dote d'une enceinte doublée d'un fossé qui enclot une surface de quinze hectares. Avec la perte du statut de capitale d'Aregenua-Vieux, entre la fin du III ${ }^{\mathrm{e}}$ siècle et le début du Iv ${ }^{\mathrm{e}}$ siècle, Bayeux intègre et contrôle désormais le territoire de sa voisine (Gauthier, Fixot, 1996, p. 37-48).

La ville poursuit son développement en devenant siège épiscopal au premier Moyen $\hat{A} g e^{2}$, tandis que s'étend hors les murs un suburbium comportant des églises de cimetière (Saint-Exupère, Saint-Vigor, Saint-Loup) et des oratoires de route (Saint-Martin-des-Entrées, Saint-Germain-dela-Lieue). Elle accueille probablement une implantation saxonne dont la présence dans le Bessin est attestée par Grégoire de Tours (Historia Francorum, V, 26 et X, 9; Béziers 1773, XXII-XXIII; Pluquet, 1829, p. 9).

Bayeux, première ville libérée de France, le 7 juin 1944, au lendemain du débarquement des forces alliées sur les côtes de la Manche, n'a pas eu à subir les vagues de bombarde-

1. Doucet 1881-1882; 1882; Lambert 1824; 1825. Une seconde intervention a été dirigée par F. Delacampagne rue Laitière en 1986-1987, dans la cour de l'ancien hôtel des Postes (Delacampagne, 1986; 1987; 1997a).

2. Bien que la tradition fasse remonter la présence d'un premier évêque à Bayeux au Iv ${ }^{\mathrm{e}}$ siècle, il faut attendre 538 et le concile d'Orléans pour disposer de l'attestation de la présence d'un prélat au sein de l'agglomération (Gauthier et Fixot, 1996, p. 37-48). 
Figure 1 : Localisation de la cité des Baïocasses. Service archéologie, conseil général du Calvados.

Figure 1: Location of the city of the Baiocasses.

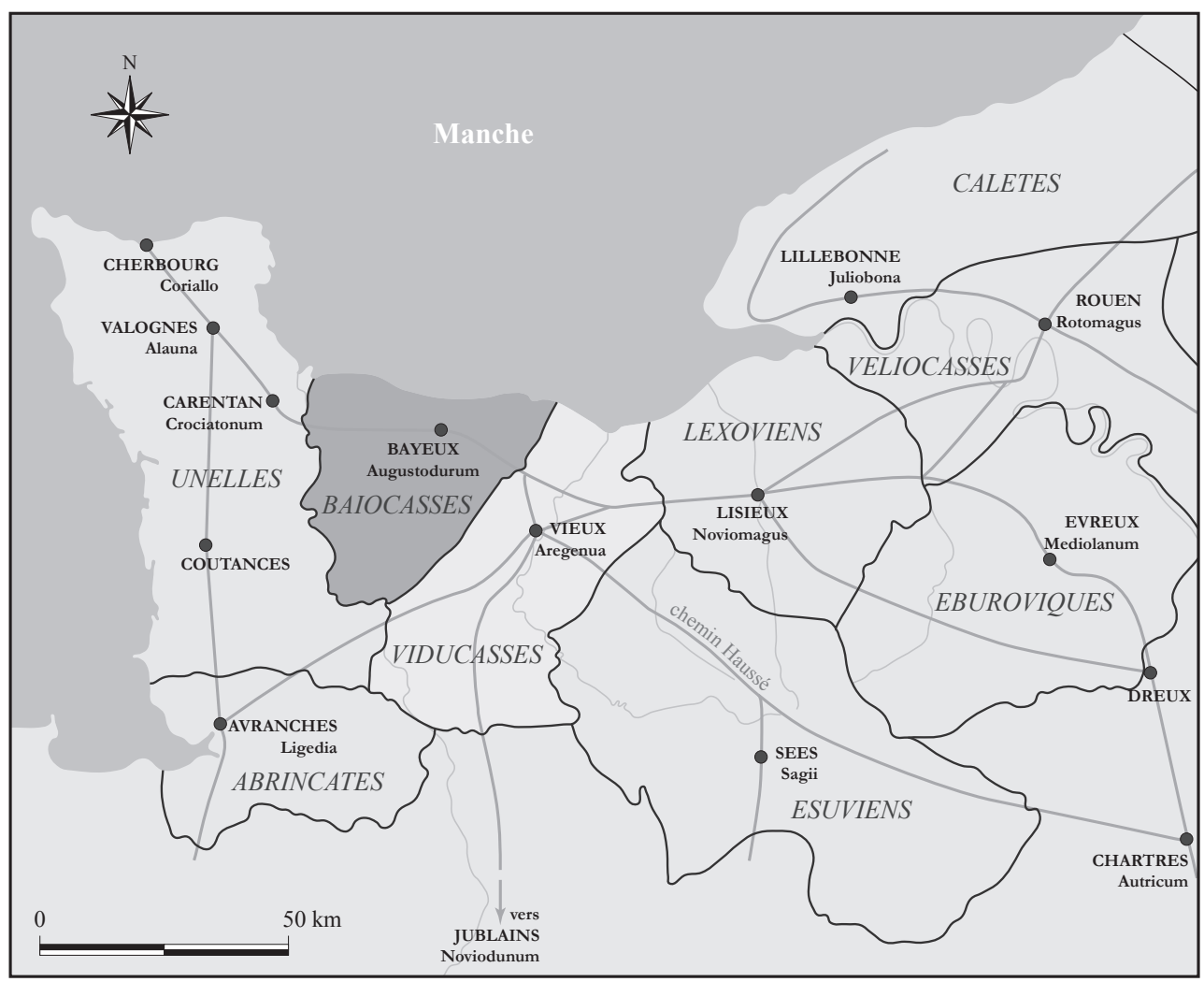

ments qu'ont connues de nombreuses villes normandes. Il en résulte une conservation du bâti ancien dans le cœur de l'agglomération avec un parcellaire assez resserré et souvent protégé en raison de sa valeur patrimoniale. Cet état de fait induit une documentation archéologique provenant essentiellement de découvertes ponctuelles. Celles-ci sont issues d'opérations archéologiques programmées ou préventives réalisées depuis les années 1980 et, plus largement, de découvertes anciennes et fortuites à l'instar de l'ensemble de blocs sculptés antiques exhumés au XIX ${ }^{e}$ siècle près de la cathédrale.

\section{Des mentions nombreuses}

Les éléments lapidaires qui sont l'objet de cette étude sont mentionnés pour la première fois dans un article du Bulletin Monumental publié en 1851 et intitulé « Excursion archéologique à la cathédrale de Bayeux ». L'auteur, Charles Bourdon, est membre de la Société française pour la conservation des Monuments. Il développe, au sein de cet article consacré à la description architecturale et archéologique de la cathédrale de Bayeux, un paragraphe intitulé "Fragments d'architecture gallo-romaine découverts auprès de la cathédrale ". L'auteur y précise que des "travaux de terrassements ", en cours au moment de la rédaction de l'article, se tiennent "autour de la cathédrale " afin de dégager et assainir l'édifice. Ces excavations ont entraîné la découverte "à une assez grande profondeur" de "fragments fort curieux d'architecture gallo-romaine" (Bourdon, 1851, p. 211). Il complète son propos par la description de certains de ces blocs, sans indiquer néanmoins leur nombre, et détaille au sein de l'ensemble la présence "de chapiteaux, de pilastres, de claveaux d'arcs en plein-cintre, de colonnes faites au tour ", autant d'éléments "d'une grande dimension" qu'il propose d'associer à des "constructions de proportions imposantes" (ibid., p. 212). L'article s'accompagne d'un dessin associant trois blocs, parfois seulement partiellement figurés (fig. 3). Ainsi pouvons-nous identifier le pilastre des deux blocs jointifs 1 et 2 (mais les autres parties des blocs ne sont pas représentées) et le bloc 4 . Le dessin s'attache ainsi plus à la représentation des rinceaux de vigne sculptés sur certains blocs qu'à une reproduction réaliste des éléments lapidaires accueillant le décor ${ }^{3}$.

3. Cette étude a été l'occasion de redécouvrir grâce à Antoine Verney, conservateur des musées de Bayeux, un ensemble de documents conservés à l'Institut national d'histoire de l'art (INHA) correspondant aux "Carnets de croquis et de notes sur des monuments de Normandie " de Ch.-É. Lambert. L'auteur y a consigné de nombreuses notes et des dessins entre 1811 et 1862. Parmi ceux-ci figurent une représentation du bloc au griffon découvert en 1828 (volume 2, pl. 43 et 44), dont il sera question ci-dessous, mais également une partie des blocs découverts en 1850-1851 (vol. 4, pl. 41, 43 et 45). Tous ces relevés, que nous reproduisons dans l'article, sont accessibles en ligne à l'adresse suivante : [www.inha.fr]. 


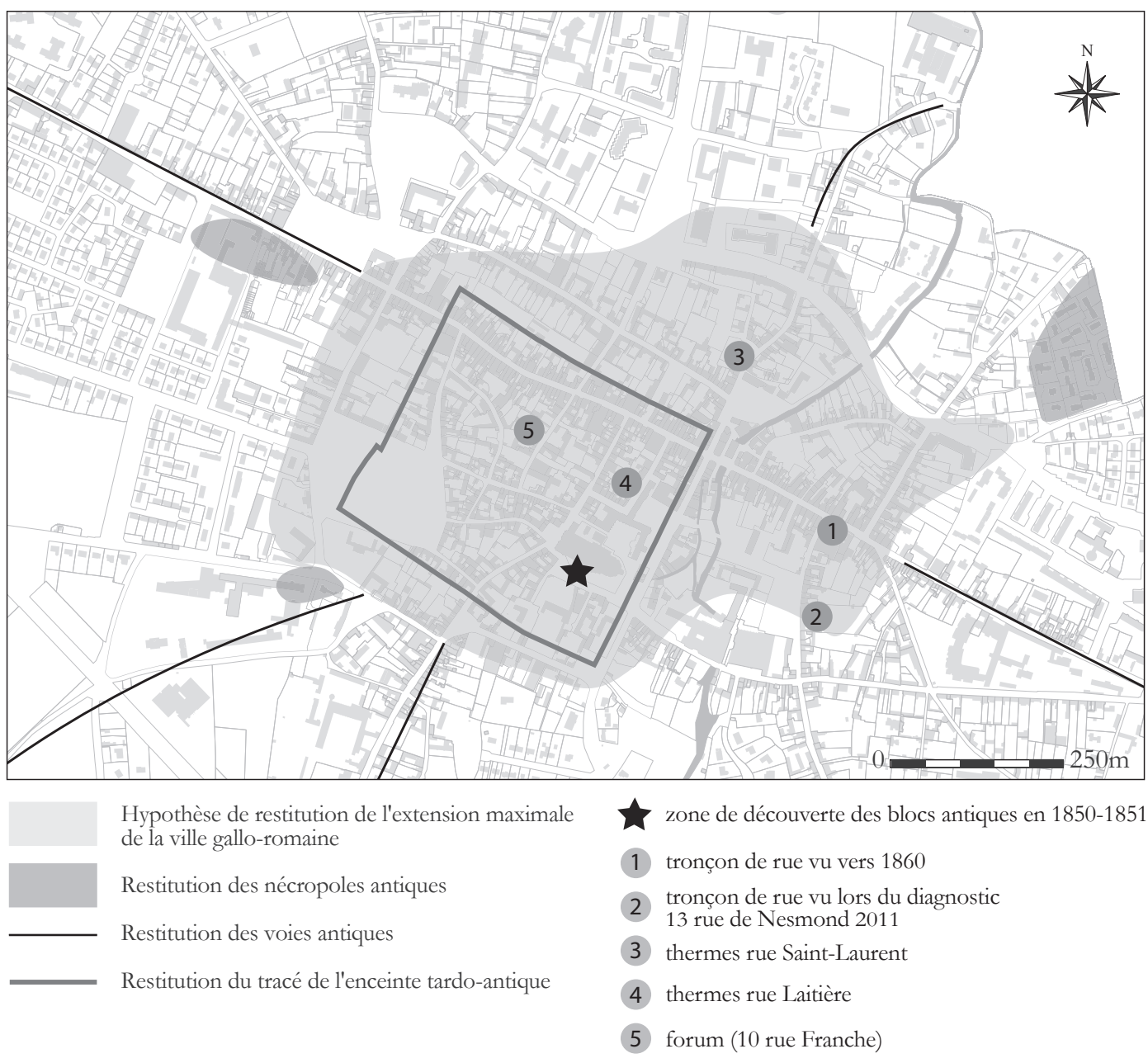

Figure 2 : Bayeux Augustodurum

(Calvados). Service archéologie, conseil général du Calvados. Figure 2:

Augustodurum (Calvados).
Cette première mention de la découverte des blocs en 1851 ou 1850 fut précédée, vingt-deux ans plus tôt, en juin 1828, par la mise au jour d'un élément sculpté lors des travaux d'installation des paratonnerres sur les tours de la cathédrale. Cette découverte est mentionnée dans une lettre adressée le 31 octobre 1829 par Charles-Édouard Lambert ${ }^{4}$, membre de la Société des antiquaires de Normandie, à Arcisse de Caumont, secrétaire de la Société. Un extrait de cette lettre est publié dans le volume des années 1829-1830 des Mémoires de la Société des Antiquaires de Normandie (Lambert, 1829-1830, p. 331-335). L'auteur relate la réalisation de quatre sondages autour de la cathédrale ${ }^{5}$ et pré-

4. Charles-Édouard Lambert (1794-1870) est un érudit bayeusain, archéologue et bibliothécaire de la ville. Il est nommé en 1833 « conservateur de la bibliothèque publique, des diverses collections et objets d'art appartenant à la ville de Bayeux ».

5. Le premier puits, destiné "à fixer l'extrémité des conducteurs des paratonnerres ", se situe sur le côté sud de la cathédrale, "au pied d'un contrefort de la tour, contre le mur de la première chapelle latérale ". Il a livré de nombreux fragments de tuiles, briques, mortier et moellons antiques ainsi qu'un fragment de placage en marbre blanc (Lambert, 18291830 , p. 331-332). L'extension du sondage entre le puits et le mur de la cise qu'une ouverture située sur son côté sud, "vers le bas du planitre, au-dessous du calvaire ", c'est-à-dire à proximité du chevet, a livré " un claveau provenant d'un grand arc" (ibid., p. 333). Ce bloc sculpté sur trois de ses six faces porte notamment la représentation d'un griffon. Si cet élément sculpté a désormais disparu, Charles-Édouard Lambert a pris soin d'en réaliser un dessin accompagnant la publication de sa lettre ${ }^{6}$ (ibid., fig. 2, pl. XII; fig. 4 et 5). L'examen de ce relevé indique que le bloc au griffon découvert en 1828 se raccorde parfaitement à l'un des claveaux découverts en 1850 ou 1851 (bloc 14).

tour de la cathédrale a permis de mettre au jour une base de colonne ainsi qu'un mur épais. Le second sondage a livré un fragment de marbre blanc, une monnaie tardive en bronze et le bloc de claveau n ${ }^{\circ} 15$ de notre étude. Les troisième et quatrième sondages ont quant à eux été réalisés du côté nord de la cathédrale, respectivement dans la cour d'Arthenay, près de la salle du chapitre, et dans " la grande cour commune à la sous-préfecture, aux tribunaux et à la mairie " (ibid., p. 335).

6. Les dessins publiés dans l'article de 1829-1830 sont une reprise de dessins originaux réalisés par Ch.-É. Lambert dans le volume 2 de ses "Carnets de croquis et de notes sur des monuments de Normandie (18261835)", pl. 43 et 44 [www.inha.fr]. 

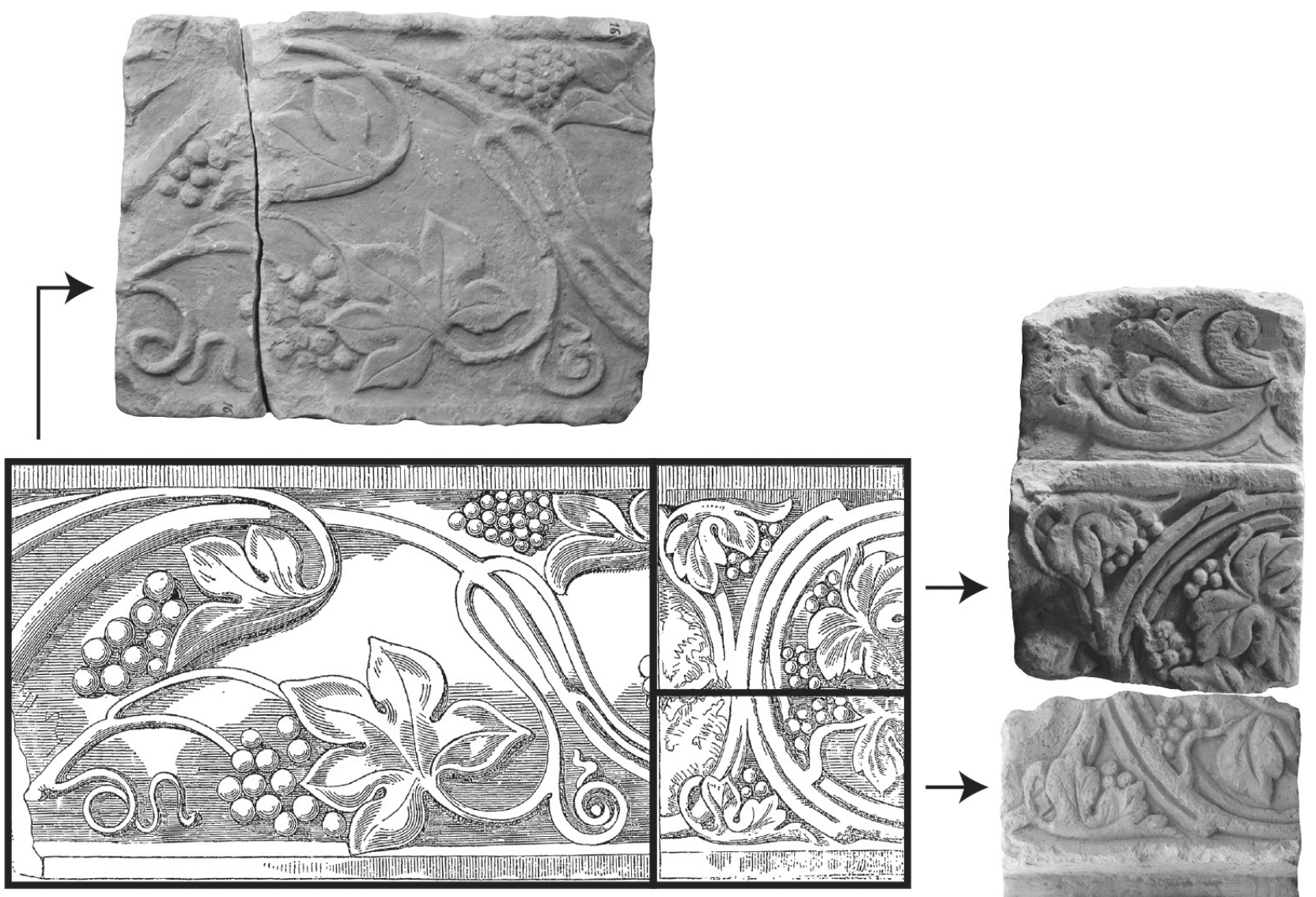

Figure 3 : Évocation publiée dans Bourdon 1851 du décor des blocs découverts en 18501851 à travers la reproduction du bloc 4 et d'une partie des blocs 1 et 2 . Service archéologie, conseil général du Calvados.

Figure 3: Evocation published in Bourdon in 1851 of the decor of the blocks discovered in 18501851 using the reproduction of block 4 and part of blocks 1 and 2 .

Il sera ensuite fait mention de cet ensemble de blocs sculptés dans plusieurs publications dont certaines apporteront quelques précisions. Il en est ainsi en 1857 dans le tome 3 de la Statistique monumentale du Calvados par Arcisse de Caumont (1857, p. 453-459) : l'auteur mentionne la découverte des différents blocs quelques années plus tôt («quantité considérable de blocs de grand appareil [...] dont plusieurs étaient sculptés "; ibid., p. 453) et y voit le témoignage de l'existence d'un édifice public important sous la cathédrale. L’article est accompagné de plusieurs illustrations : le dessin préalablement publié en 1829 , le relevé du décor du pilastre conservé sur le bloc 1 et le dessin de la face principale des assises superposées d'une colonne engagée 7 (Caumont, 1857, p. 454-455; fig. 6a et b).

7. Arcisse de Caumont identifie ces deux blocs jointifs à un chapiteau (Caumont, 1857, p. 459) comme le fera également Léon Heuzey (1869, p. 1-6).
En 1869, ces deux blocs jointifs feront l'objet d'un article intitulé "Le Dieu Mên à Bayeux» dans la Revue archéologique (Heuzey, 1869, p. 1-6, pl. 1 et couverture; fig. 6c). L'auteur est un archéologue français, membre de l'École française d'Athènes et conservateur au musée du Louvre. Célèbre pour ses missions scientifiques et ses recherches menées en Macédoine, il crut reconnaître dans le personnage debout figurant sur les blocs 5 et 6 de Bayeux le dieu Mên, divinité orientale d'origine phrygienne assimilée à la déesse Luna. Cette identification, qui ne résiste pas à l'examen critique du décor sculpté de ces deux blocs ${ }^{8}$, vint vraisemblablement à l'esprit de Léon Heuzey d'autant plus aisément que la sculpture est en mauvais état et que l'archéologue, faisant fi des décors des autres blocs, s'est laissé tromper par les ressemblances, évidentes à ses yeux, avec d'autres sculptures

8. René Norbert Sauvage, en 1910, préférait voir en cette sculpture la représentation du dieu gaulois Cernunnos (Sauvage, 1910, p. 8). 


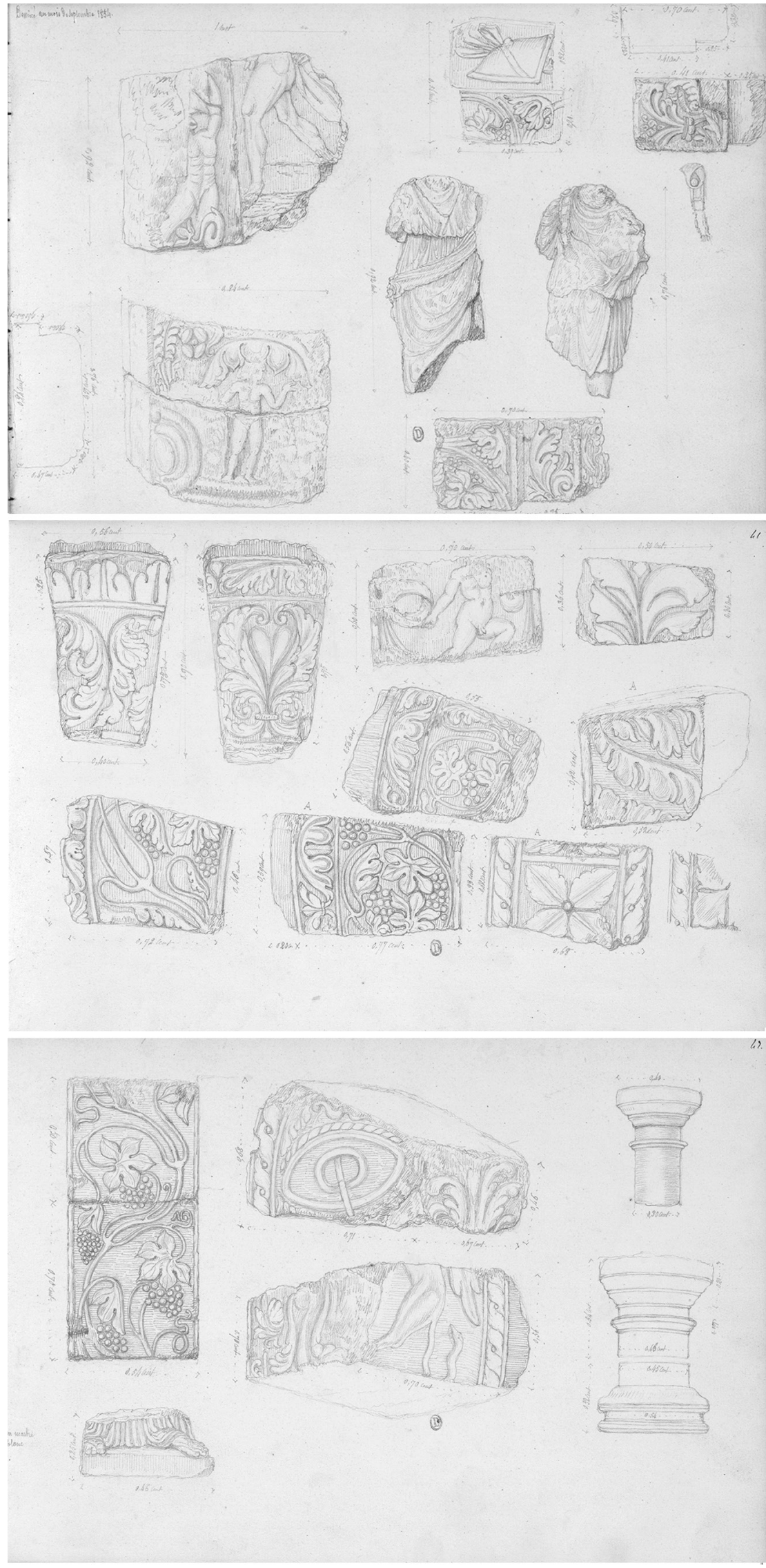

Figure 4 : Dessins du bloc au griffon découvert en 1828 figurant sur les planches $n^{\text {os }} 43$ et $44 \mathrm{du}$ volume 2 des " Carnets de croquis et de notes sur des monuments de Normandie " de C.-E. Lambert (Ms 228, INHA). Service archéologie, conseil général du Calvados.

Figure 4: Drawings of the Griffin block discovered in 1828 from plates 43 and 44 of the second volume of "Carnets de croquis et de notes sur des monuments de Normandie" by C.-E. Lambert (Ms 228, INHA). 


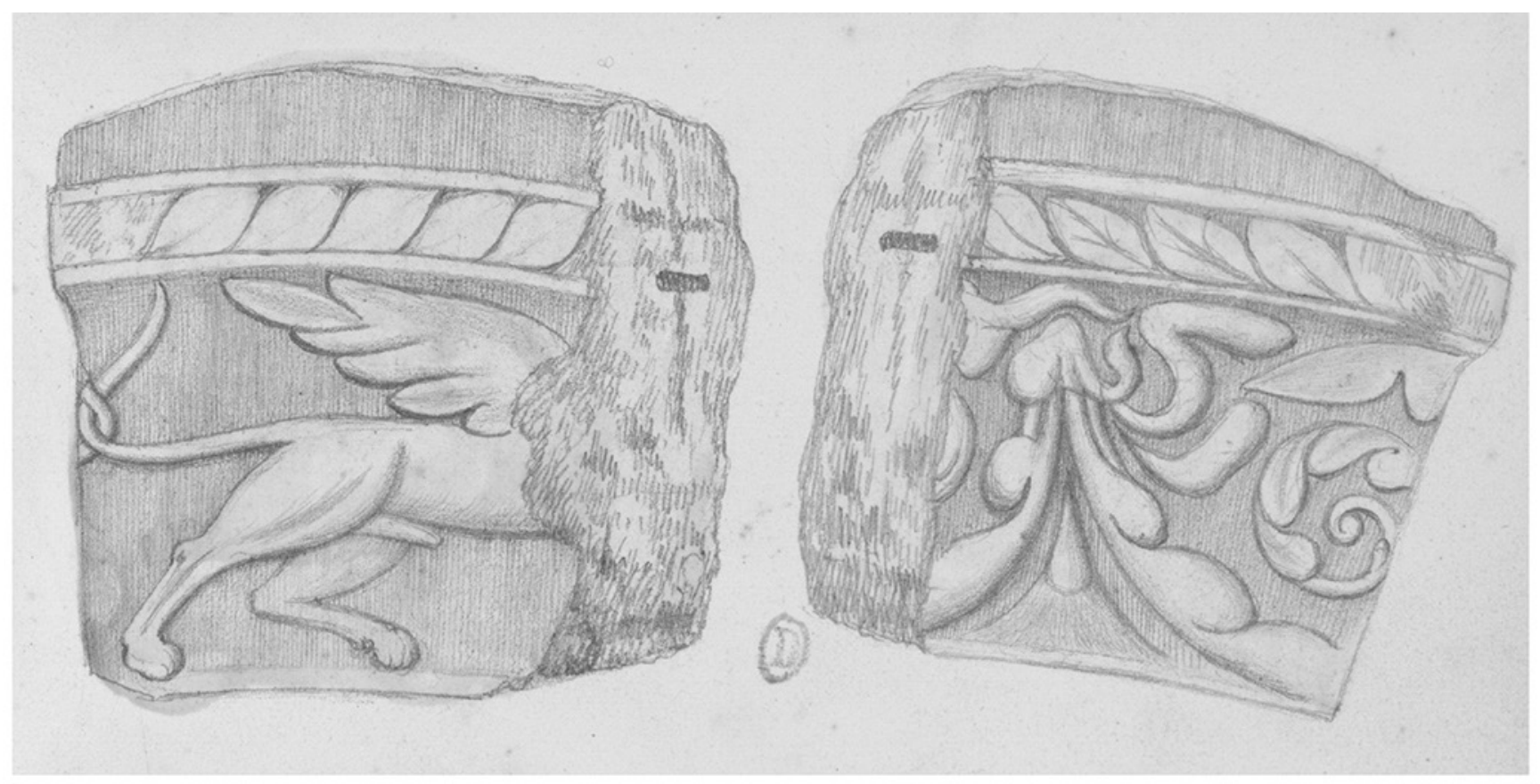

Figure 5 : Reproduction des planches $\mathrm{n}^{\text {os }} 41,43$ et 45 figurant dans le volume 4 des " Carnets de croquis et de notes sur des monuments de Normandie " de C.-E. Lambert (Ms 228, INHA). Service archéologie, conseil général du Calvados.

Figure 5: Reproduction of plates 41, 43 and 45 from the fourth volume of "Carnets de croquis et de notes sur des monuments de Normandie" by C.-E. Lambert (Ms 228, INHA).

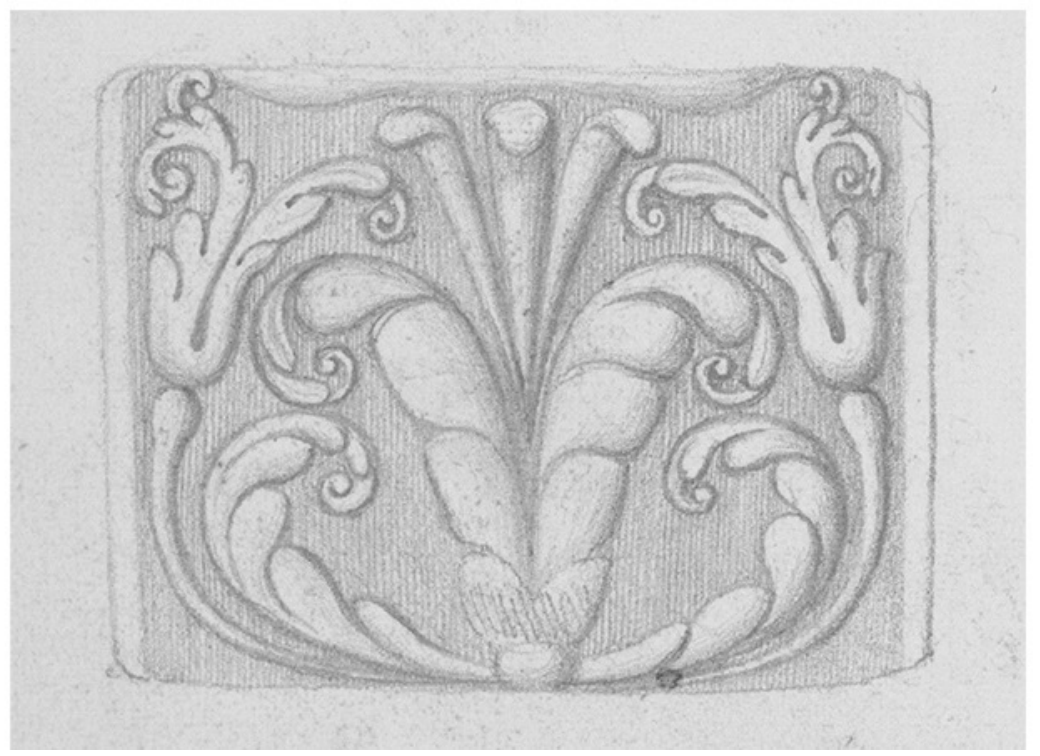

qu'il avait rencontrées, comme il l'indique lui-même', lors de missions archéologiques sur le site de Philippes, dans le nord de la Grèce.

Les blocs sculptés du planître sont ensuite mentionnés par Paul de Farcy en 1879, qui les attribue à " un arc de triomphe de la belle époque des Antonins" (Farcy, 1879, p. 207), puis par Georges Huard, qui signale l'existence « d'environ vingt-

9. À propos du décor sculpté : «J'ai cherché à la reproduire par un croquis fort imparfait, mais qui a cependant le mérite de ne représenter que ce j'ai vu sur la pierre " (Heuzey, 1869, p. 2). À propos du dieu Mên que l'auteur croit reconnaître : "Il m'était d'autant plus facile de reconnaître son image, que je l'avais rencontrée il y a peu d'années, sur les rochers de la Thrace, dans l'enceinte de la ville de Philippes " (Heuzey 1869, p. 3). cinq morceaux trouvés aux environs de la cathédrale vers 1850 et paraissant provenir d'un arc de triomphe " (Huard, 1917 , p. 367). Ils ont fait l'objet d'un inventaire dans le cadre d'un mémoire de maîtrise soutenu à Caen en 1981 (Quétier, 1981, p. 79-100). S'il pâtit d'un vocabulaire non normalisé et ignore toute approche comparative, ce travail nous a été très utile pour une première approche de la série, alors qu'elle était conservée dans des conditions qui compliquaient son étude. Pour être complet, précisons que le volume de la Carte archéologique de la Gaule consacré au département du Calvados fait très brièvement mention des blocs (Delacampagne, 1990b, p. 33), comme le Document 

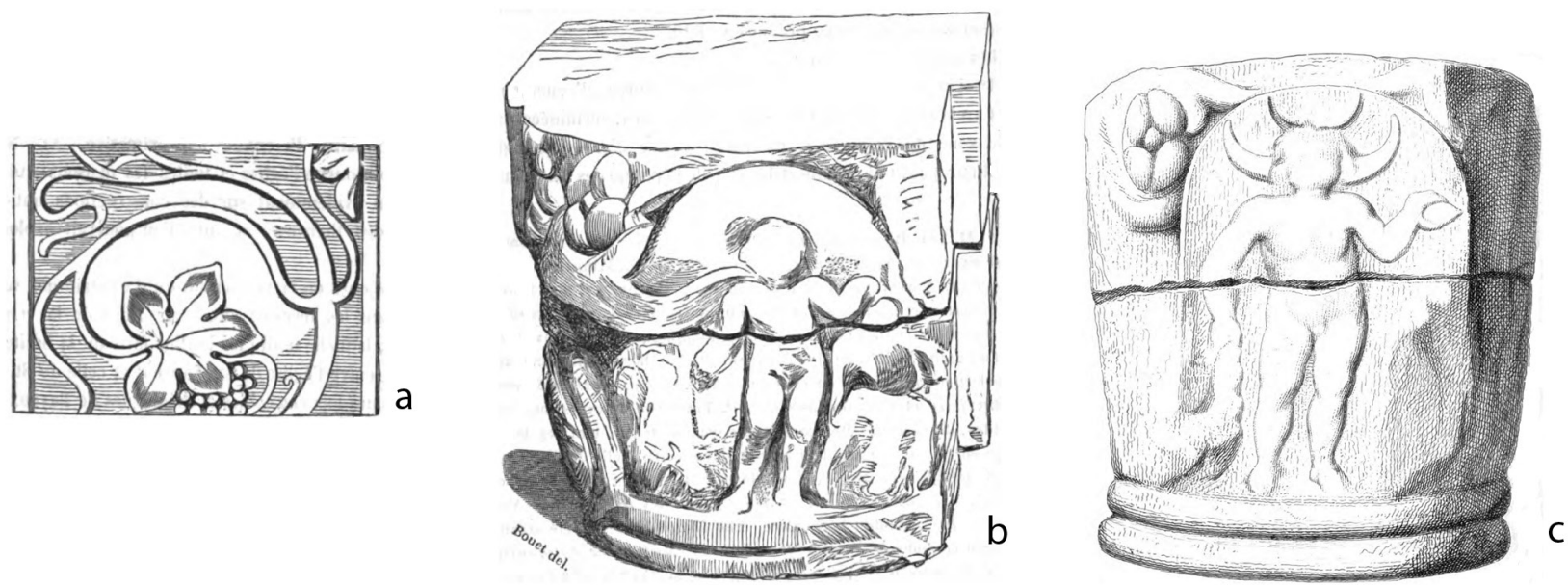

Figure 6 : Dessin du bloc 1 (à gauche) et des deux blocs superposés 5 et 6 (au centre) publiés dans Caumont 1857, p. 454-455. À droite, dessin des deux blocs superposés 5 et 6 publié dans Heuzey 1869, p. 6. Service archéologie, conseil général du Calvados.

Figure 6: Drawing of block 1 (left) and the two superimposed blocks 5 and 6 (centre) published in Caumont 1857 p. 454-455. To the right, drawing of the two superimposed blocks 5 and 6 published in Heuzey 1869, p. 6.

d'évaluation du patrimoine archéologique des villes de France (Delacampagne, Paillard, 1992, p. 17).

\section{Les conditions de découverte des blocs sculptés en 1850-1851}

En dépit de ces nombreuses mentions, les conditions de mise au jour des blocs restent floues. La première imprécision concerne leur date de découverte. Dans l'article de 1851 du Bulletin monumental, Ch. Bourdon fait état de travaux en cours ayant entraîné la découverte des blocs (" On s'occupe en ce moment de faire autour de la cathédrale des travaux de terrassement qui ont pour objet de dégager et d'assainir cet édifice ") (Bourdon, 1851, p. 211). Â défaut d'une date précise, nous pouvons envisager de fixer la découverte l'année de publication de l'article, c'est-à-dire en 1851, ou en 1850 si l'on prend en compte un court laps de temps possible entre la rédaction et la publication.

Si la localisation de la découverte reste très vague dans l'article de 1851 (" autour de la cathédrale »), Arcisse de Caumont apporte une information importante en localisant les blocs au sud de la cathédrale, au " niveau de la petite place située au Midi de la basilique " (Caumont, 1857, p. 453). La zone concernée serait donc limitée par la cathédrale au nord, l'actuelle rue Lambert-Leforestier au sud, une ligne nord-sud tangente à l'extrémité du chevet vers l'est et une seconde tangente à la façade principale de la cathédrale à l'ouest. Plusieurs éléments permettent de restreindre ce secteur entre le portail latéral sud à l'ouest et l'extrémité du chevet à l'est (fig. 7). Les blocs ont été découverts lors

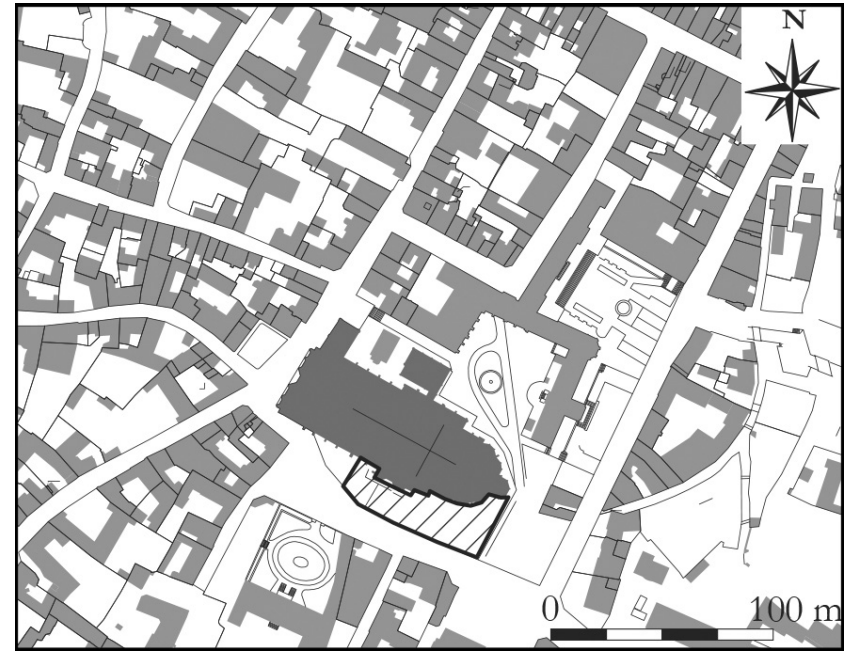

Figure 7 : Localisation de la zone de découverte des blocs sculptés de 1850-1851. Service archéologie, conseil général du Calvados. Figure 7: Location of the discovery of the sculpted blocks in 18501851.

de terrassements destinés à dégager la base des murs de la cathédrale. Les articles précités indiquent que les fondations de celle-ci sont plus profondes au niveau du chevet qu'au niveau du parvis, en accord avec le fort pendage du bascôté sud de l'édifice, passant de $48 \mathrm{~m}$ NGF près du parvis à $42 \mathrm{~m} \mathrm{NGF}$ au niveau du chevet. Les phénomènes de remblaiement étant traditionnellement plus importants en bas de pente qu'au sommet, la probabilité de la réalisation des travaux d'assainissement et de dégagement au milieu du 
XIX ${ }^{e}$ siècle est plus forte dans la partie orientale du planître que dans sa moitié occidentale. Cette hypothèse peut, de plus, être corroborée par le lieu de la découverte en 1829 du bloc orné d'un griffon près du chevet de la cathédrale (Lambert, 1829-1830, p. 333-334).

Enfin, le nombre de blocs découverts au milieu du XIX ${ }^{\mathrm{e}}$ siècle n'est jamais exprimé clairement. Seul G. Huard parle d'environ vingt-cinq éléments (Huard, 1917, p. 367). C. Bourdon (1851, p. 212) mentionne explicitement des " colonnes faites au tour ", qui sont conservées et ne sauraient être attribuées au monument qui nous retient; ce sont donc vingt blocs que nous pouvons retenir ici.

\section{Le contexte archéologique}

Plusieurs découvertes ponctuelles nous renseignent sur l'occupation du secteur de la cathédrale pendant l'Antiquité. Nous pouvons citer la mise au jour d'un fragment de fût de colonne romaine en 1825 lors du creusement dans la crypte de la cathédrale de la tombe de l'évêque Duperrier (Villers 1882 , p. 2). De nombreux fragments de briques, de tuiles, de céramiques ainsi que des monnaies des $\mathrm{III}^{\mathrm{e}}$, $\mathrm{IV}^{\mathrm{e}}$ et $\mathrm{V}^{\mathrm{e}}$ siècles ont également été retrouvés lors de terrassements effectués en 1900 pour la construction de la nouvelle sacristie sur le bas-côté nord de la cathédrale (Sauvage, 1909, p. 506). À cela s'ajoute la découverte en 1990 , lors des travaux d'assainissement du bas-côté nord de la cathédrale et de la salle capitulaire, d'un mur semi-circulaire appartenant à un édifice romain (fig. 8). L'élévation, conservée sur huit assises, présentait un parement en petit appareil calcaire maçonné à la chaux, enduit de mortier de tuileau à sa base et reposant sur un remblai contenant de la céramique du $\mathrm{I}^{\mathrm{er}}$ siècle (Delacampagne, 1990a, p. 33; 1997b, p. 8-11). Enfin, des sondages ponctuels réalisés dans le cadre de diagnostics archéologiques et d'une fouille programmée au niveau du bas-côté sud de la cathédrale et de l'hôtel du Doyen, hôtel particulier situé en vis-à-vis de la cathédrale le long de la rue Lambert-Leforestier, ont révélé un secteur très densément occupé à l'époque romaine ${ }^{10}$ (Delacampagne, 2003; 2007; Le Cloirec, 2006).

À ces données, il faut ajouter le passage à proximité de l'enceinte tardive, érigée à la fin du III ${ }^{\mathrm{e}}$ siècle - début du $\mathrm{IV}^{\mathrm{e}}$ siècle, et dont une portion est conservée. Le mur oriental

10. Les travaux de consolidation par E. Flachat, au milieu du XIX ${ }^{e}$ siècle, de la tour centrale de la cathédrale qui menaçait de s'effondrer ont donné lieu à de nombreux terrassements sous la croisée du transept pour renforcer les fondations des piliers. Ceux-ci ont notamment été décrits par $\mathrm{H}$. de Dion et L. Lasvignes en 1861 dans un rapport intitulé "Cathédrale de Bayeux. Reprise en sous-œuvre de la tour centrale " (Dion, Lasvignes, 1861). S'il n'y est pas fait référence de découvertes de vestiges archéologiques, plusieurs planches semblent indiquer la présence de substructions voire de niveaux de sols sous la cathédrale (pl. II, III et IX).

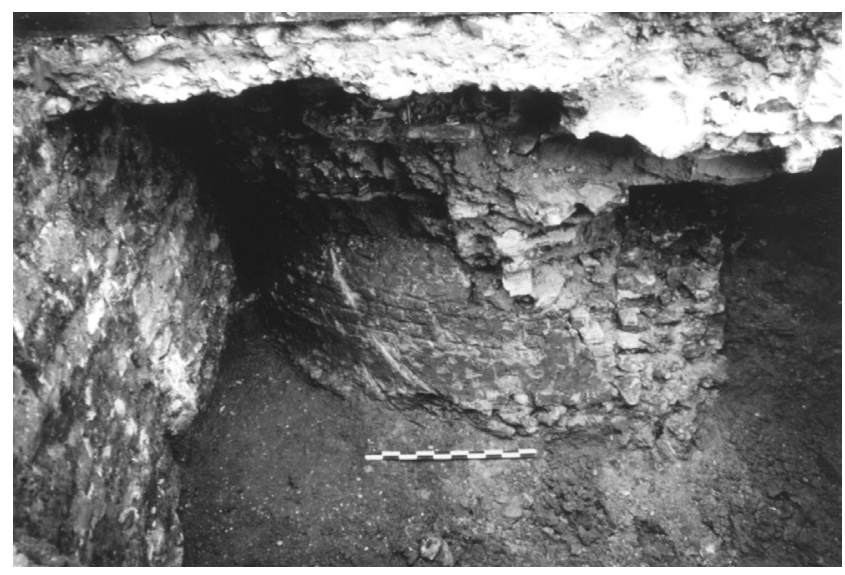

Figure 8 : Mur gallo-romain semi-circulaire observé lors de travaux le long du bas-côté nord de la cathédrale en 1990. Service archéologie, conseil général du Calvados.

Figure 8: Semi circular Gallo-roman wall observed during works on the northern side aisle of the cathedral in 1990.

de la fortification respecte une orientation nord-sud et passe à quelques mètres du chevet de la cathédrale, parallèlement à l'actuelle rue Larcher ${ }^{11}$. L'enceinte était dotée, au niveau du croisement des rues Lambert-Leforestier et Larcher, au moins à partir du Moyen Âge, d'une porte, appelée porte de l'Évêque ou porte Saint-Vigoret (fig. 9) ${ }^{12}$.

La présence sur plusieurs blocs du corpus $(12,16,18$, 19 et peut-être 9) de mortier de chaux ou de tuileau recouvrant une partie du décor sculpté et les traces de retaille de plusieurs autres éléments (blocs 7, 9 et 20) indiquent que ces derniers ont été remployés (fig. 10). On pense naturellement au castrum construit vers la fin du $\mathrm{III}^{\mathrm{e}}$-début du $\mathrm{IV}^{\mathrm{e}}$ siècle, dont un tronçon passe à proximité immédiate du lieu de découverte. Néanmoins, ce que nous savons de la mise au jour des blocs ne correspond pas à la description du démontage d'un tronçon du rempart ${ }^{13}$. Il apparaît que les éléments sculptés ont dans un premier temps été déplacés pour être réutilisés, vraisemblablement dans la construction du rempart; dans un second temps, ils durent être une nouvelle fois déplacés lors du démantèlement de la fortification à l'époque moderne ou au cours de ses nombreux réaménagements au Moyen Âge et remblayés le long du bas-côté sud de la cathédrale.

11. Le tracé de cette dernière reprend l'emplacement des anciens fossés de la ville, comblés à partir du XviII ${ }^{e}$ siècle.

12. Cette porte reliait la ville intramuros au faubourg sud-est et à la chapelle Saint-Vigoret par l'intermédiaire du pont Notre-Dame qui permettait de franchir le fossé de la ville, en partie irrigué par la rivière l'Aure. Détruite en 1756, elle est mentionnée dès la première moitié du XII ${ }^{\mathrm{e}}$ siècle (Bourrienne, 1902-1903, n 480).

13. Bourdon 1851, p. 211-212: « Les blocs de pierre équarris ou sculptés qui ont été retirés du milieu de décombres immenses [...]. » 


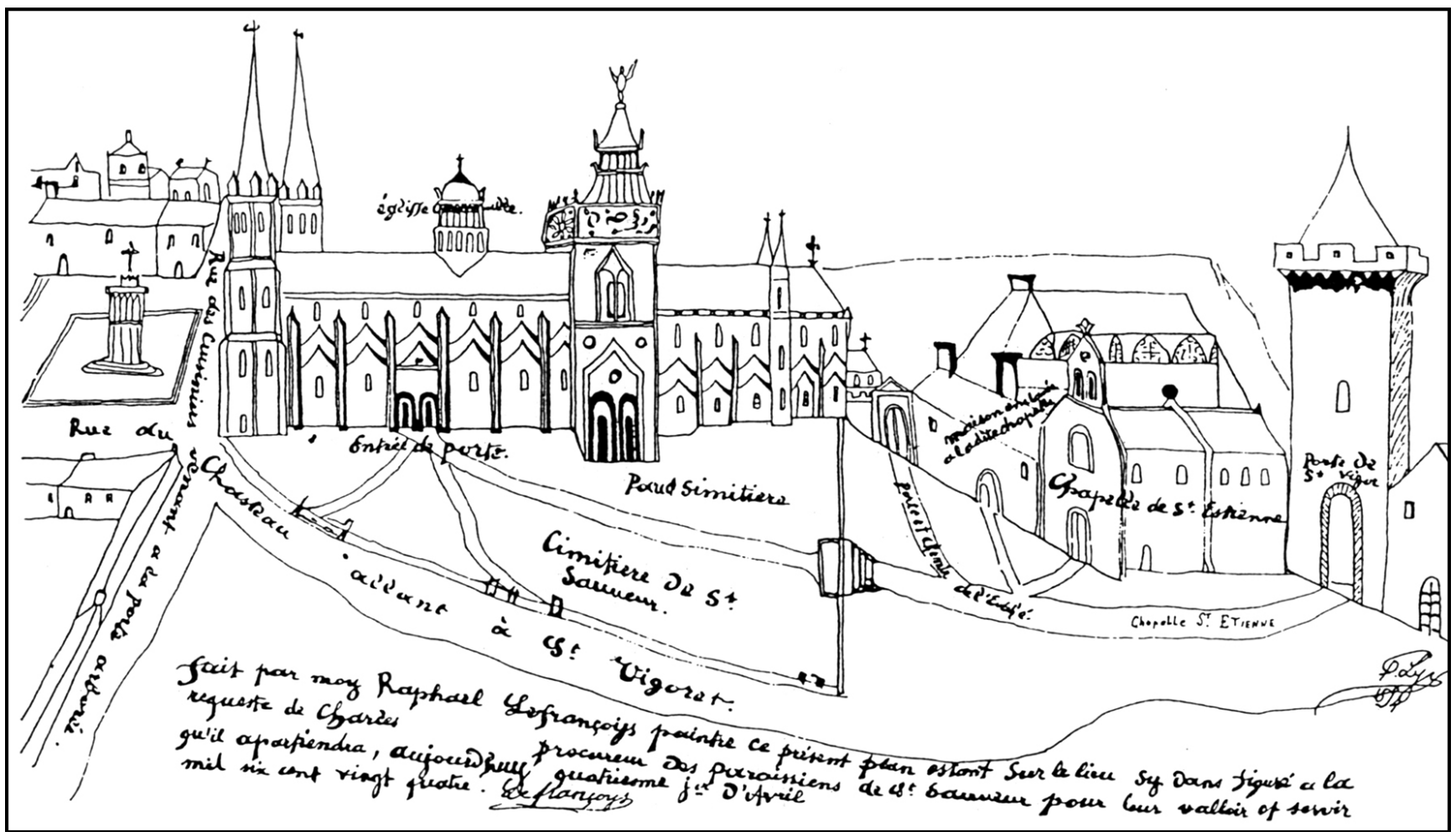

Figure 9 : Dessin de Raphaël Lefrançois représentant le bas-côté sud de la cathédrale et son environnement (1624). Service archéologie, conseil général du Calvados.

Figure 9: Drawing by Raphaell Lefrançois showing the southern aisle of the cathedral and its surroundings (1624).

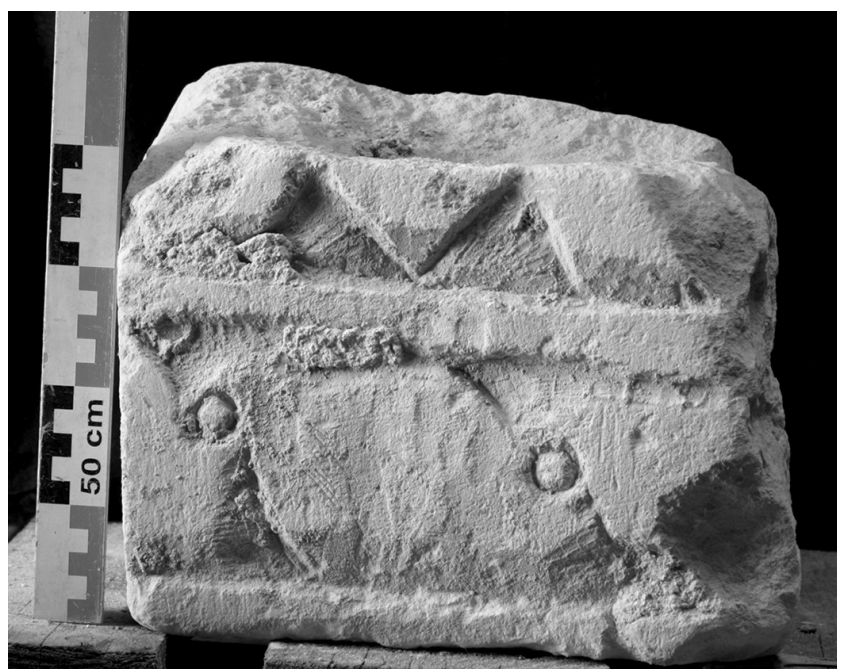

Figure 10 : Vue du bloc 12 sur lequel du mortier de chaux recouvre partiellement le décor sculpté. Service archéologie, conseil général du Calvados.

Figure 10: View of block 12 on which lime mortar partially covers the sculpted decor.

\section{INVENTAIRE DES BLOCS ATTRIBUABLES AU MONUMENT ${ }^{14}$}

Vingt blocs peuvent être rattachés à un même monument, sur la foi de considérations typologiques et stylistiques. Dix-neuf sont conservés, le dernier (bloc 15) n'étant connu que par des dessins anciens, déjà évoqués. Ces éléments peuvent être répartis en quatre catégories morphologiques : les blocs ornés de pilastres (groupe I); les blocs ornés d'une colonne engagée (groupe II); les blocs de claveaux, qui sont évidemment les plus importants pour l'identification du monument (groupe III); un bloc isolé, enfin, qu'il est difficile de situer dans le monument (type IV) ${ }^{15}$.

14. Cet inventaire obéit à une logique architecturale qui n'était pas celle des recensements précédents; aussi adoptons-nous une nouvelle numérotation. Pour faciliter la consultation croisée de cet article et des travaux antérieurs, nous avons jugé utile d'établir une table de concordances, fournissant d'abord notre numéro d'inventaire, puis la référence à l'inventaire de A.-M. Quétier (1981). $1=3 ; 2=20 ; 3=22 ; 4=16 ; 5$ et $6=4 ; 7=$ $8 ; 8=7 ; 9=6 ; 10=12 ; 11=5 ; 12=10 ; 13=27 ; 14=14 ; 15=$ absent de l'inventaire de Quétier $16=13 ; 17=18 ; 18=17 ; 19=11 ; 20=25$.

15. Conformément aux normes de la description architecturale, nous ne parlons jamais de la largeur des blocs, mais de leur longueur, de leur hauteur et de leur profondeur. Pour les claveaux, la hauteur est donnée par rapport au tracé générateur de l’archivolte. 
L'étude pétrographique conduite par Xavier Savary (géologue, service archéologie, conseil général du Calvados) a révélé que les dix-neuf blocs conservés ont été sculptés dans des calcaires de deux natures différentes. Une grande majorité d'entre eux (seize blocs) ont été réalisés en calcaire de Caen, un calcaire bathonien à grain très fin, extrait dans la région de Caen, à environ trente kilomètres à l'est de Bayeux (Calvados). Les quatre autres blocs $(5,6,14$ et 17) sont quant à eux sculptés dans un calcaire bioclastique grossier, riche en éléments très arrondis de lithoclastes, de coquilles de bivalves et de bryozoaires.

En soi, le recours à divers affleurements pour la construction d'un édifice important n'est pas surprenant, tant la pratique est bien documentée. C'est l'emploi de deux calcaires aux propriétés très différentes qui pose problème : quelle raison a poussé les sculpteurs romains à utiliser un calcaire bioclastique grossier ne permettant pas une finesse de travail équivalente au calcaire de Caen, dont les qualités physiques (homogénéité, finesse du grain, dureté relative, etc.) en font un matériau privilégié pour la sculpture? Le mauvais état de conservation des blocs de grand appareil en calcaire grossier ne permet pas aujourd'hui de vérifier d'éventuels défauts de finition. Leur situation au sein du décor du monument - deux blocs proviennent d'une archivolte ( ${ }^{\text {os }} 14$ et 17$)$ et deux autres d'une colonne engagée ( ${ }^{\text {os }} 5$ et 6 ) - ne permet pas plus d'expliquer ce choix. Une étude élargie sur la provenance des roches, en particulier calcaires, utilisées dans la construction des édifices d'Augustodurum permettrait peutêtre d'apporter une réponse à cette interrogation.

\section{Groupe I : pilastres ornés de rinceaux}

\section{Bloc 1 - Bloc de grand appareil}

orné de deux pilastres (fig. 11)

Dimensions. H.T. : $39 \mathrm{~cm}$; long. cons. : 53,1 cm; prof. cons. : $72 \mathrm{~cm}$.

Lits de pose et d'attente sont conservés; le lit de pose, que nous avons pu observer, est dépourvu d'aménagement. Ce bloc présente un plan complexe. Il conserve l'extrémité droite d'un bloc de grand appareil portant en façade un pilastre orné d'un rinceau de vigne (A), amputé de sa partie gauche; ce pilastre saille de $6 \mathrm{~cm}$ sur une plage ( $\mathrm{Lg}$ : $23,1 \mathrm{~cm}$ ) décorée d'un rinceau à feuilles acanthisées (B); la face en retour, à droite, est ornée d'un troisième rinceau (C), lui aussi acanthisé ( $\mathrm{Lg}$ : $42 \mathrm{~cm}$ ). Le bloc devait prendre place à gauche d'un passage : le rinceau de vigne soutenait l'archivolte, tandis qu'un pilastre orné en façade et sur au moins un côté encadrait le passage lui-même.
Figure 11 : Bloc 1. Plan du lit de pose (relevé, Yvan Maligorne, DAO Annabelle Cocollos, CG 14), les rinceaux A et $\mathrm{B}$, le rinceau $\mathrm{C}$. Clichés Annabelle Cocollos, CG 14.

Figure 11: Block 1. Plan of the resting surface (drawing, Yvan Maligorne, illustration Annabelle Cocollos, $C G$ 14), the scrolls $A, B$ and $C$ (photos Annabelle Cocollos, CG 14).
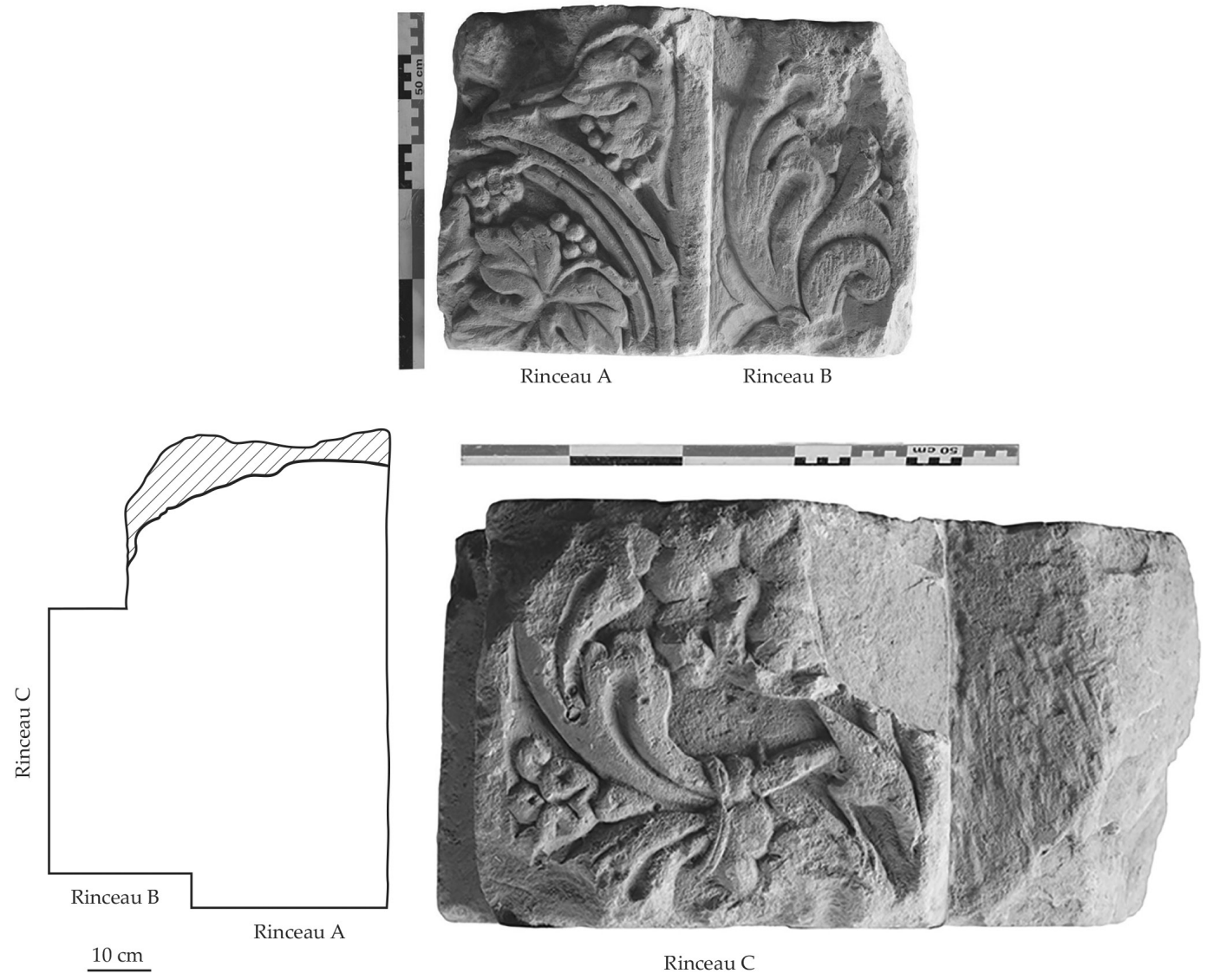
Le rinceau de vigne (A) est dense et a fait l'objet d'un traitement très plastique. La tige principale génère plusieurs tiges secondaires qui lui sont parfois strictement parallèles et qui donnent naissance à leur tour à des feuilles de vigne et des grappes de raisin.

Le rinceau (B) qui jouxte le pilastre est composé d'une succession de longues feuilles. Aucune n'est intégralement conservée, mais elles devaient comporter trois folioles : deux sont vues de face, comportent trois digitations et présentent un limbe fortement animé par une concavité centrale, encadrée par un plissement; la troisième foliole est vue de profil; elle est lisse et son limbe est plat; elle s'enroule alternativement à gauche et à droite pour livrer passage à la feuille suivante, dans une composition dont le schéma reproduit celui des files de crosses, très fréquentes en Gaule. Les deux écoinçons conservés sont meublés de calices à deux brins, l'un ouvrant sur un pistil.

Le rinceau (C) sculpté sur la face en retour se développe entre deux filets. Un caulicole courbe, dont la gaine est ornée de languettes et la collerette de trois sépales retournés, donne naissance à deux bractées et une tige rectiligne, laquelle génère un calice à deux brins s'ouvrant sur un pistil. Les deux bractées devaient être identiques, mais une seule peut être partiellement décrite : deux folioles vues de face sont conservées, dont le limbe est creusé d'une concavité en cuiller et la bordure découpée en trois courtes digitations. La bractée supérieure présente une courbure inverse à celle du caulicole, et devait à son tour livrer passage à un caulicole générant à nouveau deux bractées, l'une ascendante et constituant le tracé principal du rinceau, l'autre tombante et générant un gros fleuron, conservé et constitué d'une corolle de pétales dentelés autour d'un cœur en pistil. Sur le caulicole est penché un petit échassier.

L'utilisation d'une acanthe de type voisin sur B et C témoigne de l'appartenance des deux faces à un même élément, en l'occurrence le pilastre bordant le passage.

\section{Bloc 2-Bloc de grand appareil orné d'un pilastre (fig. 12)}

Dimensions. H.T. : $38,8 \mathrm{~cm}$; $\mathrm{Lg}: 55,5 \mathrm{~cm}$ dont $34,5 \mathrm{~cm}$ pour la plage ornée du bouclier et $22 \mathrm{~cm}$ pour le pilastre; le pilastre fait saillie de $6,7 \mathrm{~cm}$ sur la zone du bouclier; prof. max. : $80 \mathrm{~cm}$ avec le pilastre.

Les lits de pose et d'attente sont conservés; seul le dernier nous a été accessible : il est dépourvu d'aménagement.

La face de parement conserve, à droite, une partie d'un pilastre orné d'un rinceau de vigne, faisant saillie sur un panneau sculpté d'un bouclier. Sont conservés une partie de la tige principale et de tiges secondaires, les vestiges d'une feuille de vigne vue de face, une feuille posée de profil meublant un écoinçon, et deux petites grappes de raisin. Le relief est bordé à gauche par un listel.
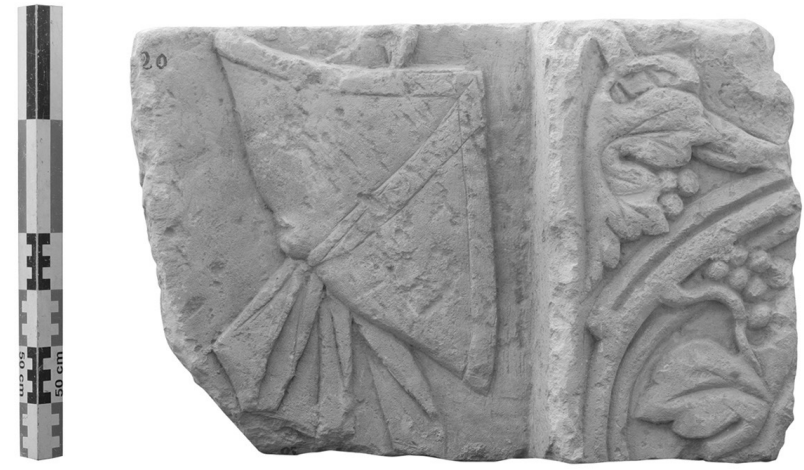

Figure 12 : Bloc 2, la plage ornée d'un bouclier suspendu et le pilastre décoré d'un rinceau. Cliché Annabelle Cocollos, CG 14. Figure 12: Block 2, the pannel decorated with a suspended shield and the pilaster decorated with a scroll.

Sur le panneau de gauche, qui présente un fond concave, le bouclier vu de profil est bordé d'un listel; il présente un umbo hémicylindrique. On peut essayer d'en déterminer la typologie : fortement bombé, le bouclier devait présenter de face un grand côté courbe dont les extrémités sont reliées par deux petites concavités; il s'agit donc d'une des variantes de la pelta, le bouclier d'amazone. Il est suspendu par deux rubans, dont une faible portion est visible en haut; ils entourent le bouclier et sont noués en bas, une boucle et leurs deux extrémités étant bien conservées. On notera une petite erreur du sculpteur, la bordure du bouclier restant visible sous le ruban qui devrait la masquer.

\section{Bloc 3 - Bloc de grand appareil orné d'un pilastre (fig. 13)}

Dimensions. H.T. : 48,6 cm; $\mathrm{Lg}$ du pilastre : $56 \mathrm{~cm}$; $\mathrm{Lg}$ de la partie lisse (bûchée?) : $64 \mathrm{~cm}$; le pilastre fait saillie de $9,9 \mathrm{~cm}$ à droite et $18,2 \mathrm{~cm}$ à gauche; profondeur de la queue du bloc : $77,7 \mathrm{~cm}$.

Le lit de pose est conservé, dressé au marteau taillant, dont les traces en gerbes sont bien visibles, et au ciseau. La face arrière est très endommagée, en particulier à l'endroit où pouvait se situer un pilastre correspondant à celui de la face antérieure; aucune trace de décor n’y est conservée.

Le bloc est très endommagé, et seul peut être décrit le pilastre qui orne la partie gauche de la façade. Il est orné d'un rinceau de vigne entre deux filets : traité en bas-relief, il est très aéré; la tige principale donne naissance à trois tiges secondaires, dont deux partent symétriquement; l'une engendre une feuille de vigne vue de profil, l'autre une feuille vue de face; une autre tige s'en échappe qui donne naissance à une grappe. Enfin, deux tiges s'enroulent en vrille, sans porter ni feuille, ni grappe.

À droite du pilastre s'étend une grande plage lisse. Un examen attentif montre qu'une partie au moins de la surface 
Figure 13 : Bloc 3. (a) La face dont le décor est partiellement conservé. (b) Le revers. Clichés Annabelle Cocollos, CG 14).

Figure 13: Block 3. (a) The face on which the decor is partially preserved. (b) The back.
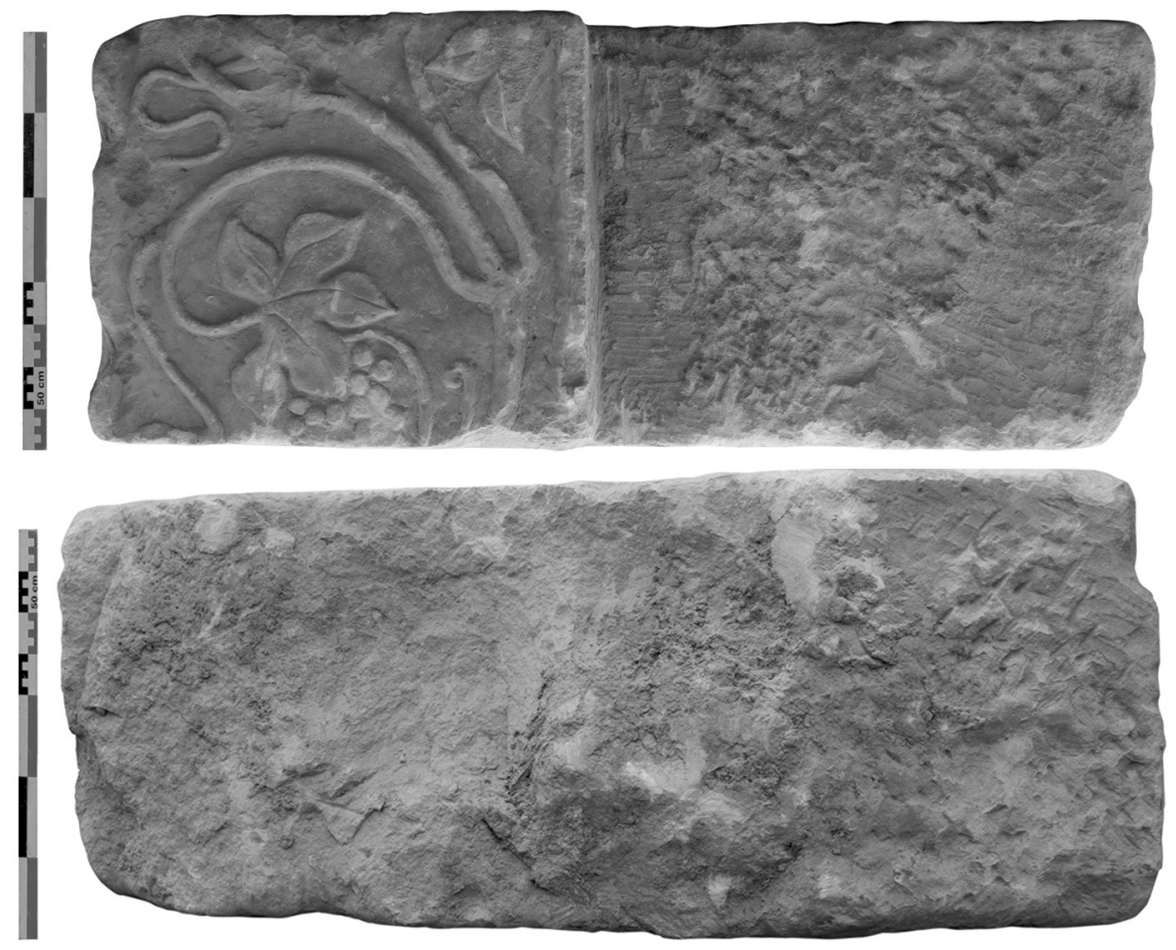

a été bûchée : elle offre encore un léger relief et on y décèle des traces de broche et de ciseau. Cette zone au traitement grossier est entourée de traces très nettes de ciseau à large tranchant ou de marteau taillant. Le fond des reliefs étant bien dressé sur tous les autres blocs, il s'agit sans doute de traces de retaille.

\section{Bloc 4-Parement d'un pilastre (fig. 14)}

Dimensions. H.T. : $72 \mathrm{~cm}$; Lg : $56 \mathrm{~cm}$; épaisseur : $11,5 \mathrm{~cm}$.

Le bloc a été scié, de façon à ne conserver que le parement du pilastre; il est constitué de deux fragments jointifs.

Le pilastre est orné d'un rinceau de vigne encadré par deux filets. La tige principale dessine d'amples ondulations, meublées par des tiges secondaires, qui, à un endroit, en jaillissent symétriquement. Les tiges secondaires peuvent se subdiviser à leur tour, une des tiges engendrant alors une feuille ou une grappe, l'autre formant une vrille.

Les quatre blocs de pilastres conservés se rattachent à deux schémas, manifestement servis par des " mains » différentes ${ }^{16}$. Le schéma le plus simple est représenté par les blocs 3 et 4 , jointifs (fig. 15) : nous observons ici un rinceau simple, aux ondulations régulières et amples, dont la tige principale, apparemment ininterrompue, génère des tiges secondaires nombreuses, lesquelles peuvent se subdiviser à

16. Pour la typologie des rinceaux de pilastre, voir Mathea-Förtsch 1999, p. 8-10.

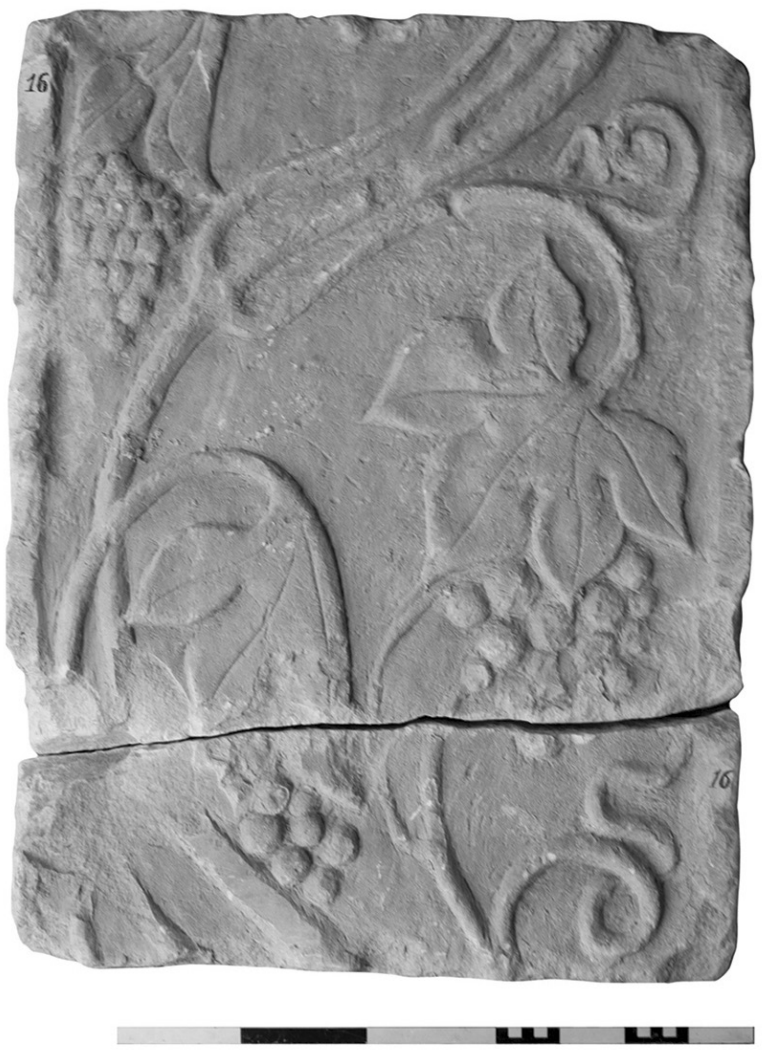

Figure 14 : Bloc 4, deux fragments jointifs d'un pilastre. Cliché Annabelle Cocollos, CG 14.

Figure 14: Block 4, two joined fragments of a pilaster. 


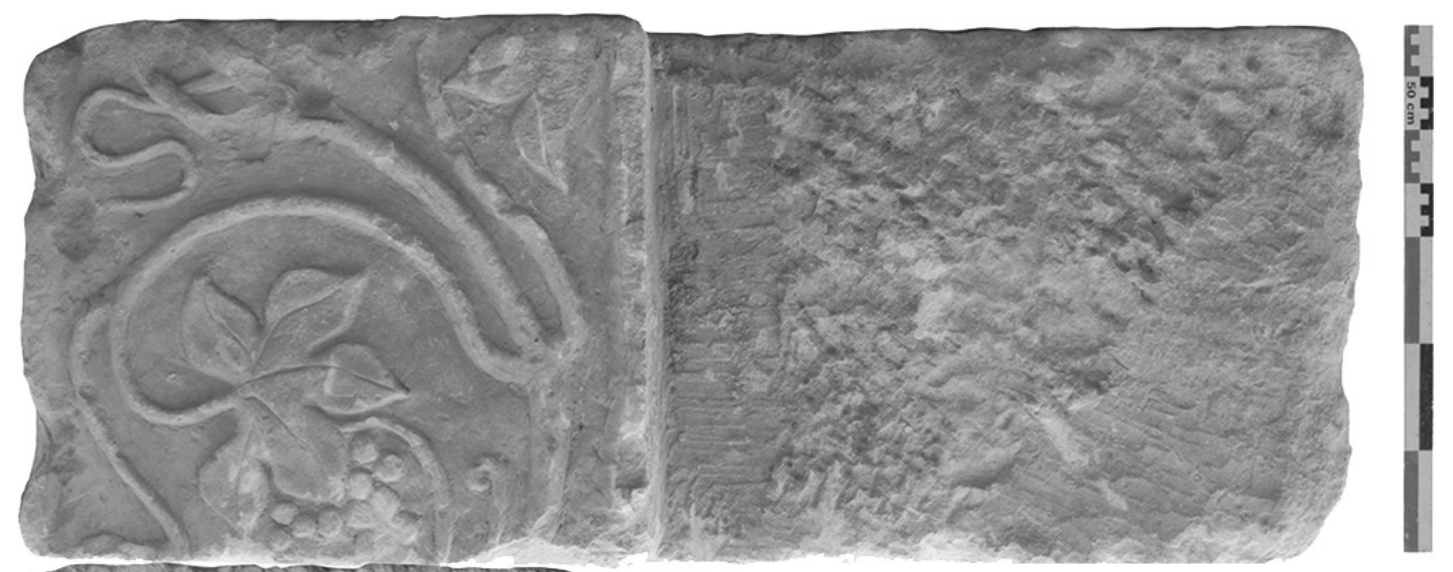

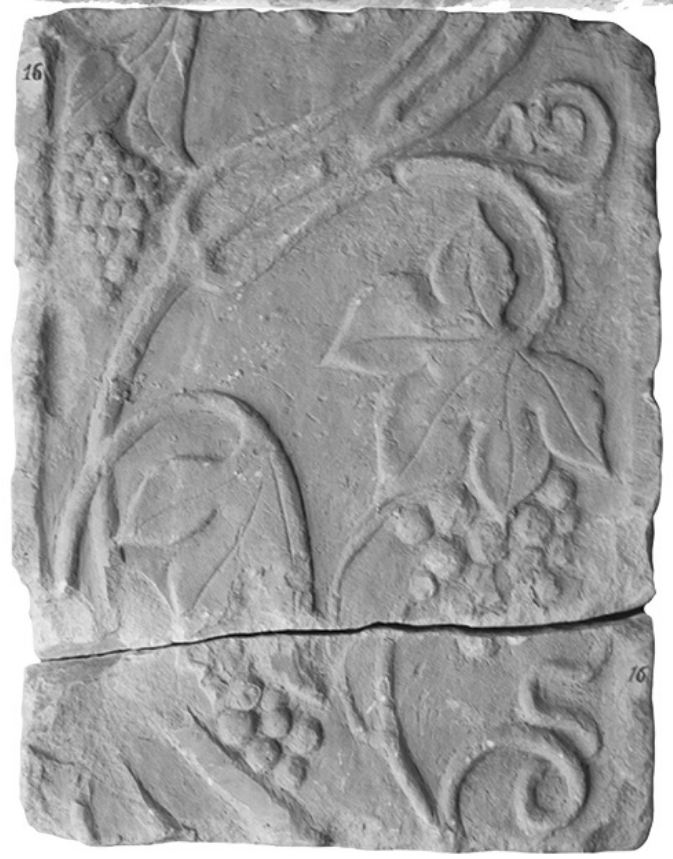

Figure 15: Les blocs 3 et 4. Clichés et DAO Annabelle Cocollos, CG 14.

Figure 15: Blocks 3 and 4.

leur tour; elles donnent naissance à des feuilles de vigne, à des grappes, ou s'enroulent en vrille. Les feuilles, tantôt vues de profil, tantôt posées à plat, comptent cinq folioles parcourues par une nervure axiale; elles recouvrent presque systématiquement une grappe de raisin. Le trait saillant du rinceau est la récurrence des " tridents " formés par la tige principale et deux tiges secondaires qui s'en écartent symétriquement.

Les blocs 1 et 2, eux aussi jointifs (fig. 16), illustrent un second schéma, dans lequel deux tiges symétriques se croisent, dégageant des médaillons circulaires meublés par des feuilles de vigne posées à plat recouvrant des grappes. Les écoinçons sont quant à eux occupés par des feuilles vues de profil enveloppant une grappe de raisin; les deux écoinçons qui se font face portent des motifs exactement symétriques. Le sens de lecture ne va pas sans poser problème : celui que nous adoptons est destiné à conférer un caractère ascendant aux rinceaux acanthisés du bloc 1, puisqu'un rinceau descendant serait tout à fait incongru; mais le système d'attache du bouclier du bloc 2 invite à une lecture inverse, les boucles des rubans étant généralement situées au-dessus de l'objet suspendu. Ces hésitations n’ont cependant aucune incidence sur la structure du rinceau de vigne.

Autant que par le dessin, les deux ensembles se distinguent par le traitement. Le premier présente une composition aérée; le fond est très présent, le relief faible et le rendu des végétaux peu plastique; les folioles ont des bords lisses et leur nervure est réduite à une étroite incision. Le second type développe un décor beaucoup plus dense, rendu de manière plus plastique; le relief est plus accusé, les feuilles aux bords dentelés sont plus souples et animées par des nervures plus larges et plus profondes.

\section{Groupe II : blocs portant une colonne engagée}

Blocs 5 et $6-$ Deux assises superposées d'une colonne engagée (fig. 17)

Dimensions. Assise inférieure. H.T. : 40,2 cm; Lg face arrière : 84,3 cm; prof. de la queue du bloc : $37,2 \mathrm{~cm} ; \mathrm{H}$. des jambes : $24,3 \mathrm{~cm}$. Assise supérieure. H.T. : $34,9 \mathrm{~cm}$; corde de la colonne : $70 \mathrm{~cm}$; flèche de la colonne : $26,5 \mathrm{~cm}$; Lg de la partie parallélépipédique : $84,5 \mathrm{~cm}$; prof. de la partie parallélépipédique : $42,2 \mathrm{~cm}$ à droite.

Le lit d'attente conserve une partie d'un trou de louve, tronqué dans le sens longitudinal par la cassure de la face arrière (longueur : 8,5 cm; profondeur : 9,9 cm).

Les deux blocs, jointifs, présentent une colonne à demi engagée dans un bloc parallélépipédique dont les faces sont très bien dressées et dépourvues de défoncé. La colonne est assez grossièrement dégagée, en particulier sur l'assise infé- 

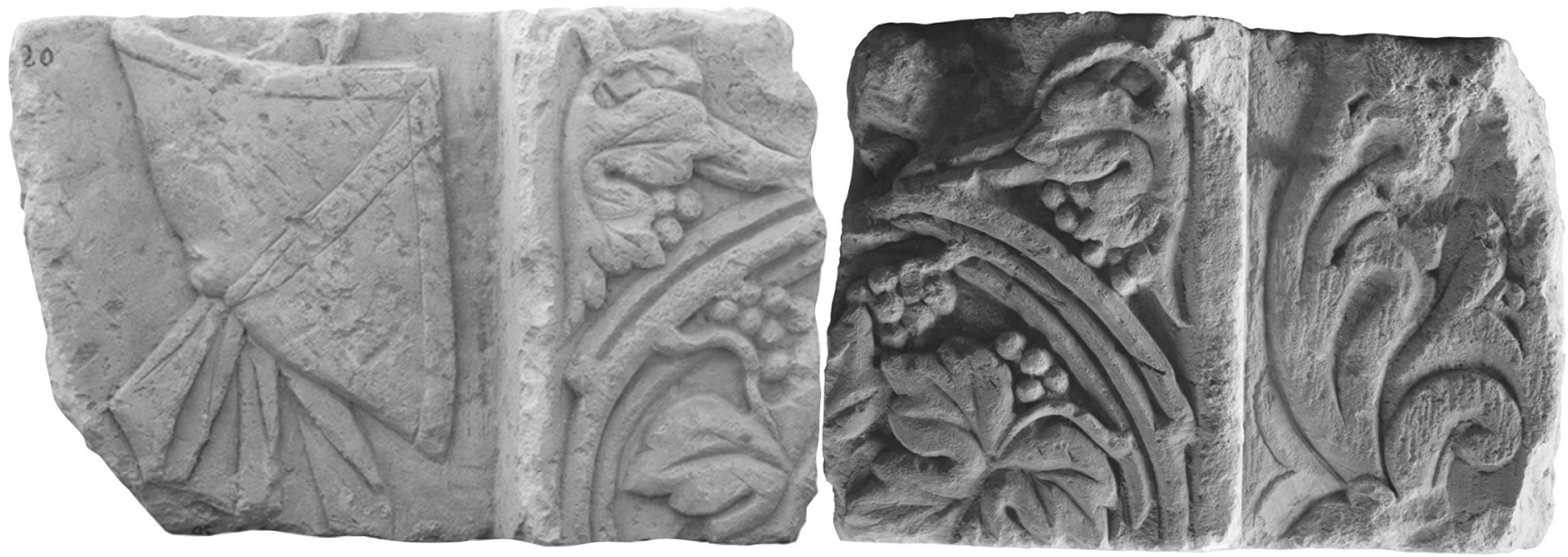

Figure 16: Les blocs 1 et 2. Clichés et DAO Annabelle Cocollos, CG 14. Figure 16: Blocks 1 and 2.

Figure 17 : Les blocs 5 et 6. (a) Vue d'ensemble des deux assises superposées. (b) Le rinceau qui s'élève à gauche du personnage. (c) La collerette végétale qui surmonte le personnage ( $\mathrm{a}$ et $\mathrm{b}:$ clichés Yvan Maligorne, c : cliché Annabelle Cocollos, CG 14). Figure 17: Blocks 5 and 6. (a) View of the superimposed blocks. (b) The rinceau to the left of the figure. (c) The vegetal collar above the figure.

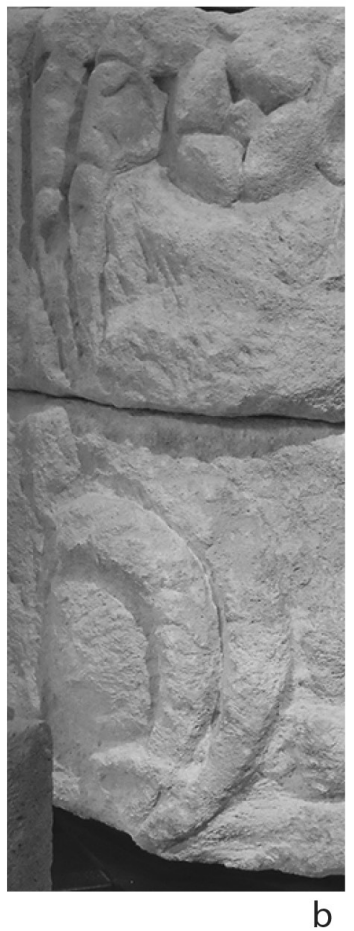

a

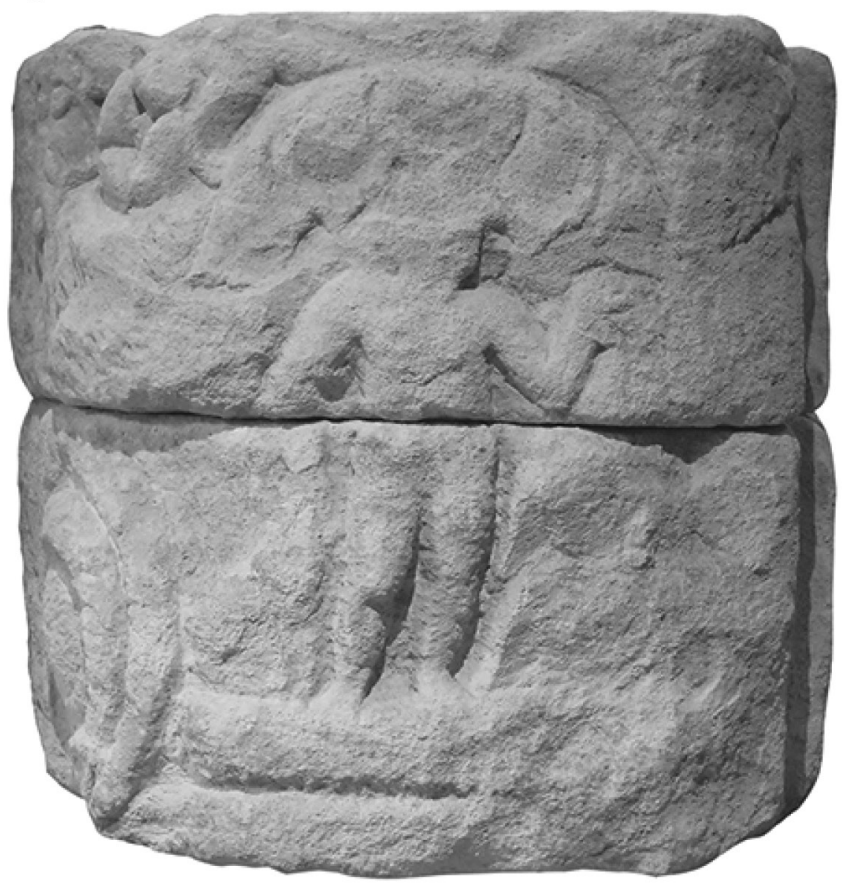

b

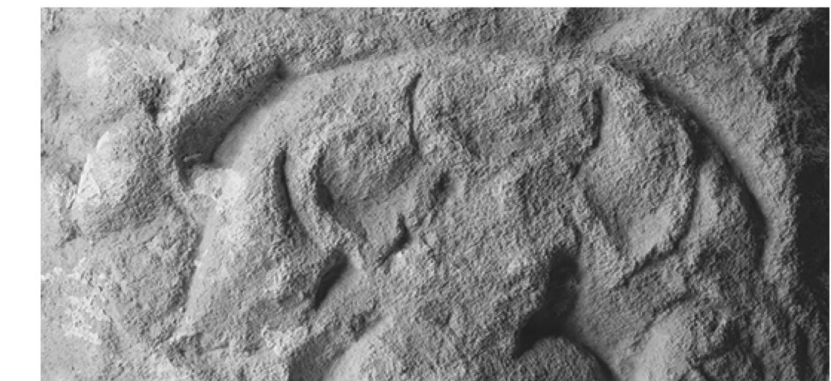


rieure : l'arrondi est irrégulier et incomplet, puisque la partie droite est presque rectiligne et n'accueille aucun décor; sur l'assise supérieure, cette partie est sommairement traitée, mais a reçu l'amorce d'un décor végétal. Il est possible que la partie droite du bloc soit demeurée peu visible, ou même qu'elle ait été accolée à une structure qui en ait compliqué la sculpture.

Le décor est très endommagé. Un homme debout se dresse, nu (fig. 17a), sous une couronne végétale composée de cinq folioles au limbe convexe dont la bordure est divisée en trois digitations (fig. 17c) : la figure est animée par un contrapposto sommaire, puisqu' elle est appuyée sur sa jambe gauche et que sa jambe droite est légèrement repliée. Le bras droit descend le long du corps et tient un objet allongé - on pourrait penser à une massue, mais encore à un bouclier vu de profil. Le bras gauche est levé et replié; la main, sommairement traitée - seul le pouce est individualisé - semble se saisir d'un objet sphérique. Le visage est bûché, mais la tête semble couverte, peut-être par un casque. Aux pieds du personnage, à gauche comme à droite, le parement est trop érodé pour qu'on puisse distinguer quoi que ce soit, mais le sujet semble inscrit dans une véritable scène, dont l'identification pose problème : l'objet allongé reposant à terre tenu de la main droite et l'objet sphérique que l'homme saisit de la main gauche nous ont d'abord incité à identifier Hercule au jardin des Hespérides, même si c'est à Atlas que revient le plus souvent la cueillette des fruits; mais il faudrait alors identifier le couvre-chef à la léonté, qu'Hercule porte le plus souvent nouée autour du cou dans les représentations gauloises. Mars, son casque et son bouclier offrent une autre possibilité, même si l'objet sphérique ne reçoit pas d'explication immédiate dans cette hypothèse.

On remarque que le personnage repose sur un double bandeau lisse, qui lui sert de socle. Il ne semble pas s'agir d'une séparation entre deux registres, à la manière des panneaux que l'on observe sur de nombreux pilastres, parce que ces bandeaux n'interrompent pas le décor végétal qui se développe à gauche. On doit donc se demander si l'on n'a pas affaire à une manière de candélabre végétal peuplé de part en part, comme cela est très fréquent, par des personnages; en ce cas, le dais végétal qui couronne le personnage pourrait être la collerette d'un caulicole dont la gaine serait masquée.

Quoi qu'il en soit de cette interprétation, un rinceau s'élève à gauche du personnage (fig. 17b). Seulement épannelé sur l'assise inférieure, il est achevé sur le bloc supérieur; il relève du type à tiges parallèles : les tiges sont engainées par des bractées épaisses, constituées de folioles tripartites comportant une large digitation axiale encadrée par deux petites digitations. Un fleuron engendré par les tiges secondaires est visible sur l'assise supérieure : très simple, il est formé de quatre pétales épais répartis autour d'un cœur en bouton.
Un volume épannelé signale l'esquisse d'un autre fleuron sur l'assise inférieure. Il était prévu que ce décor soit reproduit à droite, mais il est simplement esquissé, cette partie du bloc étant largement laissée à l'état d'épannelage.

\section{Groupe III : blocs d'archivolte}

Bloc 7-Claveau (fig. 18)

Dimensions. H.T. : $92,1 \mathrm{~cm} ; \mathrm{Lg}$ cons. : $51,5 \mathrm{~cm}$; prof. à l'intrados : 68,7 cm.
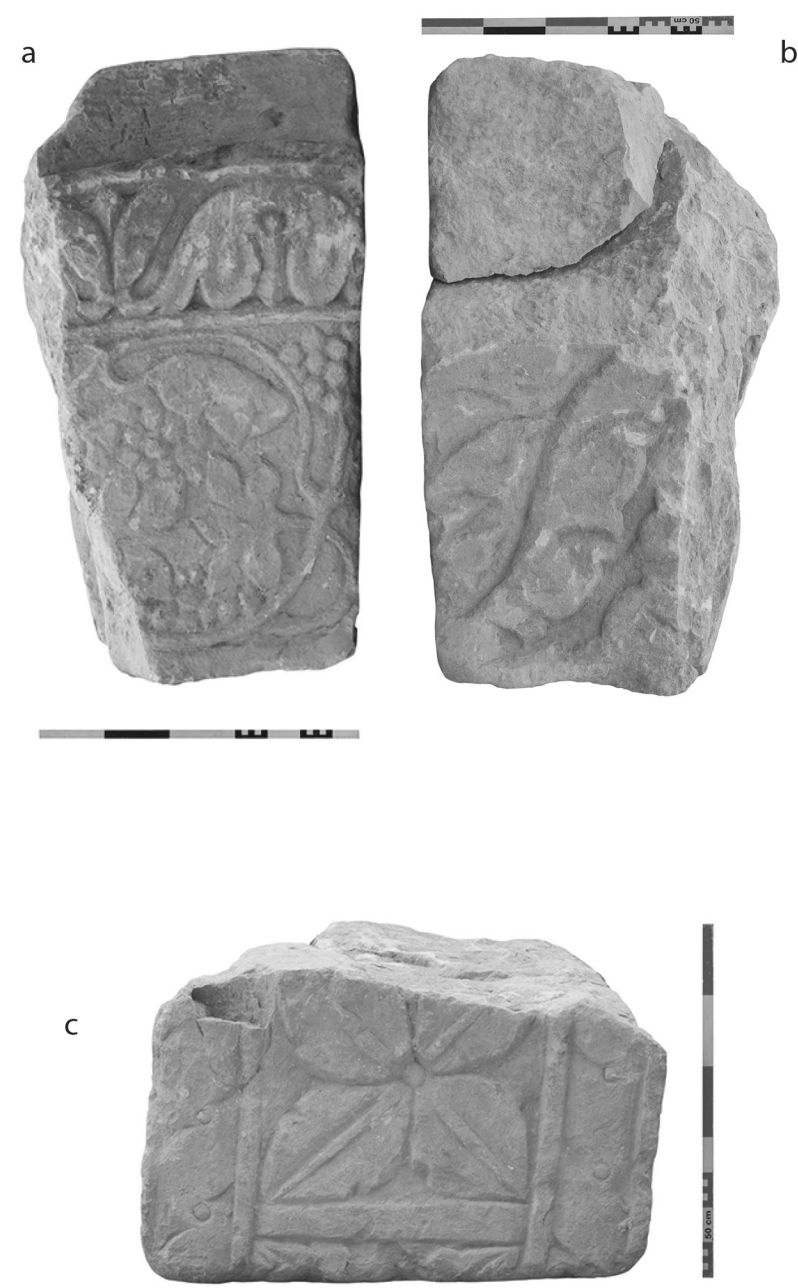

Figure 18 : Bloc 7, archivolte (cliché Annabelle Cocollos, CG 14). (a) Archivolte ornée d'un rinceau de vigne et couronnée d'un anthémion. (b) Archivolte ornée d'un anthémion. (c) Intrados. Figure 18: Block 7, archivolt. (a) Archivolt decorated with a vine scroll and crowned by an anthemion. (b) Archivolt decorated with an anthemion. (c) Intrados. 
Le bloc se compose de six fragments jointifs, l'un étant beaucoup plus important que les autres.

L'une des faces de parement est bien conservée (fig. 18a). Sur un bandeau encadré par deux listels (H. : $55 \mathrm{~cm}$ ), se développe une section d'un rinceau de vigne : la tige principale, grêle, donne naissance à trois tiges secondaires, dont deux, disposées symétriquement, dessinent un motif de trident. Ces tiges, qui se subdivisent parfois à leur tour, engendrent des feuilles de vigne, vues de face ou de profil, ou des grappes de raisin. Le couronnement de l'archivolte (H. : $22 \mathrm{~cm}$ ) est constitué d'un anthémion sur talon, coiffé d'un listel : des demi-feuilles aux bords profondément dentelés sont unies et enserrent des lancettes, dans un schéma directement emprunté au rai de cœur en ciseau; l'extrémité des feuilles se relève et passe dans un bandeau qui la lie à la feuille adjacente; les extrémités de deux feuilles contiguës constituent avec le calice qu'elles encadrent un bouquet ascendant.

L'autre archivolte est très endommagée, toute la partie supérieure et le couronnement ayant été abattus (fig. 18b). Le bandeau est orné des vestiges d'un anthémion (H. cons. : $50 \mathrm{~cm}$; Lg cons. : 39,5 cm). Au centre, est visible une feuille de liaison de profil, comportant quatre folioles à la surface très légèrement convexe se relevant le long de la bordure; une seule est dentelée. Elle relie deux bouquets : le bouquet ascendant, à gauche, a conservé trois feuilles vues de profil, traitées comme les folioles de l'élément de liaison; du bouquet tombant sont seulement visibles les extrémités de deux feuilles.

L'intrados (fig. 18c) est orné des vestiges de deux fleurons inscrits dans des caissons carrés séparés par un listel $(\mathrm{Lg}: 6 \mathrm{~cm})$. Quatre pétales lancéolés et très discrètement dentelés, structurés par une côte axiale plate, sont disposés sur la diagonale, autour d'un cœur en bouton. Les caissons étaient encadrés de chaque côté par une moulure légèrement convexe ornée d'une tresse à deux brins et bordée par deux listels $(\mathrm{Lg}: 16 \mathrm{~cm})$.

\section{Bloc 8-Claveau (fig. 19)}

Dimensions. H.T. : 72,7 cm; H. bandeau de l'archivolte : $56,5 \mathrm{~cm}$; H. couronnement : 16,2 cm; Lg de l'archivolte : $47 \mathrm{~cm}$ en bas, $56 \mathrm{~cm}$ en haut; prof. cons. à l'intrados : $65,2 \mathrm{~cm}$ (incomplète).

Les deux faces de joints latérales sont conservées, mais le bloc est retaillé au-dessus du couronnement. Une des faces de joint est creusée d'un trou de louve.

Une des archivoltes est bien conservée (fig. 19a) : elle porte un rinceau de vigne entre deux minces listels $(H$. : $56,5 \mathrm{~cm}$ ). La tige principale engendre des tiges secondaires symétriques, formant un motif en trident; l'une de ces tiges engendre à son tour une nouvelle tige. Toutes donnent nais-
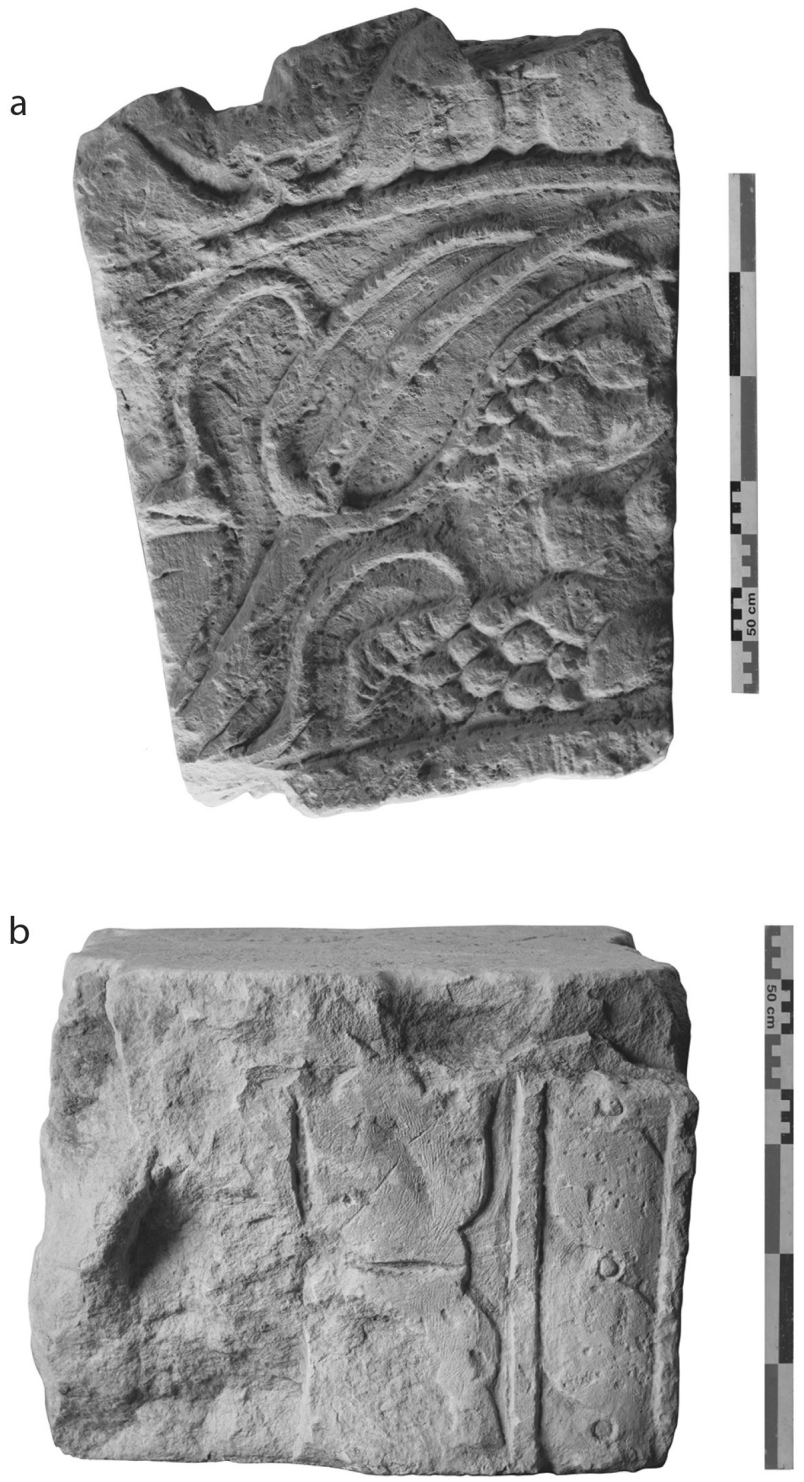

Figure 19 : Bloc 8. (a) archivolte ornée d'un rinceau de vigne. (b) Intrados. Clichés Annabelle Cocollos, CG 14.

Figure 19: Block 8 (a) decorated with a vine scroll. (b) Intrados.

sance à des feuilles ou des grappes. Le couronnement est partiellement amputé (H. cons. : $16 \mathrm{~cm}$ ) et a un profil plus proche du quart-de-rond que du talon; il est décoré d'un anthémion identique à celui du bloc 7 .

L'autre archivolte est abattue. L'intrados (fig. 19b) est décoré d'un fleuron de quatre pétales : animés par des digitations plus importantes que sur le bloc 7 , ils présentent un limbe souple, seulement structuré par une fine nervure axiale. Les caissons étaient encadrés latéralement par une tresse à deux brins sur une moulure légèrement convexe, bordée elle-même par des listels. 


\section{Bloc 9-Claveau (fig. 20)}

Dimensions. H.T. : 77,5 cm; Lg max. : $62,5 \mathrm{~cm}$; prof. au lit supérieur : $51,5 \mathrm{~cm}$.

Sur une des faces de joint (fig. 20a) sont creusés quatre trous de louve : l'un est très largement amputé en profondeur et doit être lié à la mise en place du bloc dans le monument d'origine, suivie d'une retaille en œuvre. Les autres peuvent correspondre à des remplois.
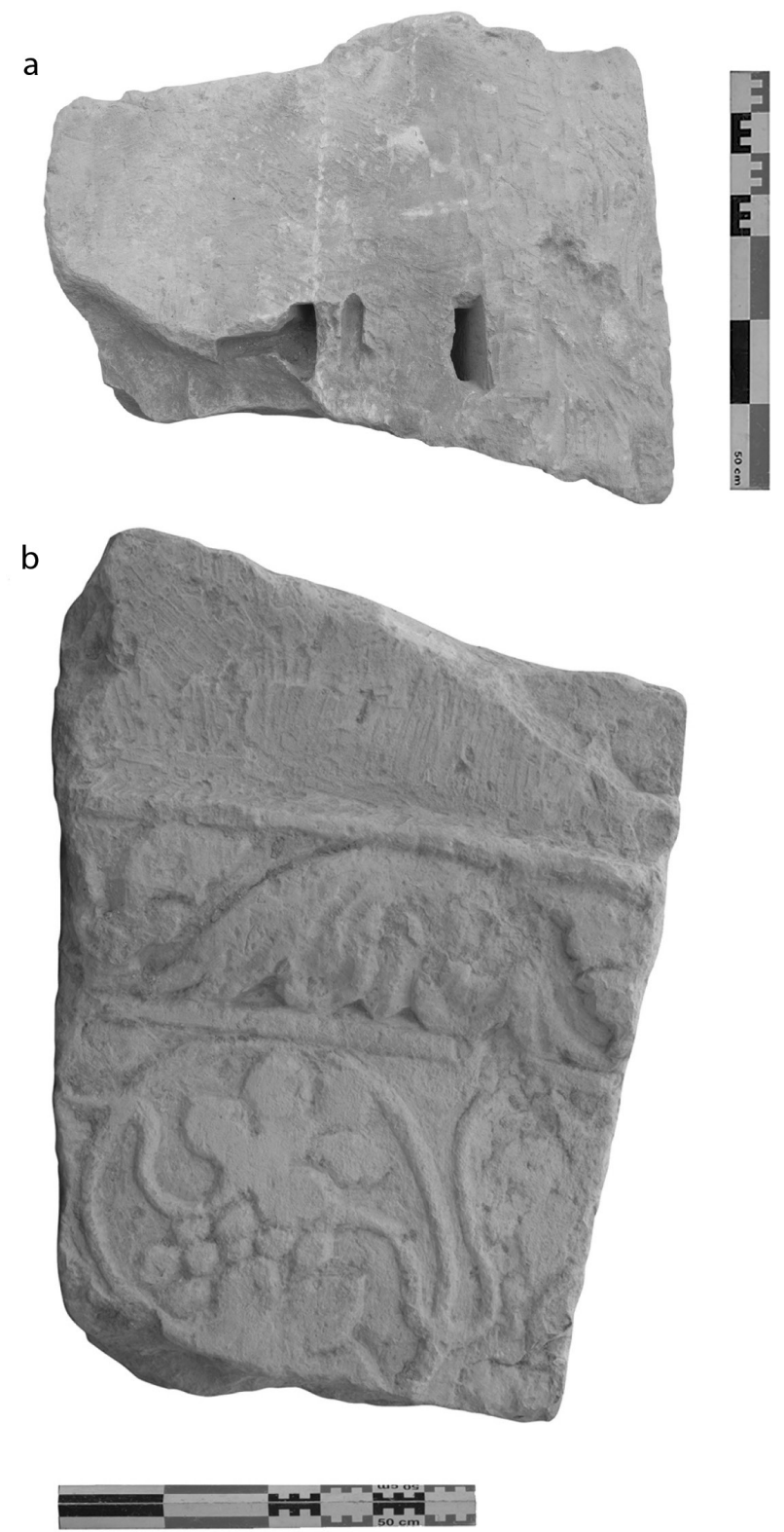

Figure 20 : Bloc 9. (a) Face de joint. (b) Archivolte ornée d'un rinceau de vigne et couronnée d'un anthémion. Clichés Annabelle Cocollos, CG 14.

Figure 20: Block 9. (a) Joint face. (b) Archivolt decorated with a vine scroll and crowned by an anthemion.
La partie supérieure d'une archivolte est conservée (fig. 20b). Le bandeau sculpté porte un rinceau de vigne (H. cons. : $35 \mathrm{~cm}$ ) sculpté en méplat et assez mal conservé; on observe une tige secondaire, qui engendre elle-même d'autres tiges et dont l'enroulement est meublé de feuilles et de grappes. Le couronnement $(\mathrm{H} .: 19 \mathrm{~cm})$ est orné d'un anthémion sur un talon, surmonté d'un listel (voir la notice du bloc 7 pour la description).

\section{Bloc 10 - Claveau (fig. 21)}

Dimensions. H.T. : $36 \mathrm{~cm}$; Lg : 53,5 cm; prof. cons. : $33,5 \mathrm{~cm}$.
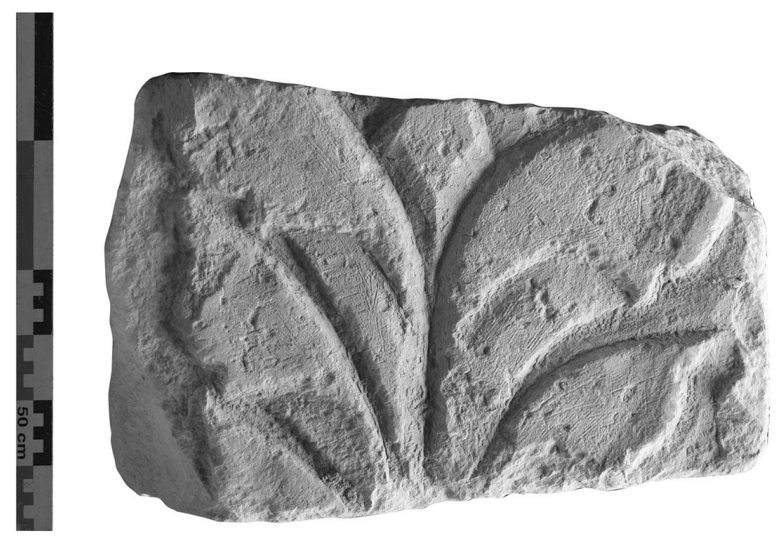

Figure 21 : Bloc 10, portion d'un claveau orné d'un anthémion. Cliché Annabelle Cocollos, CG 14.

Figure 21: Block 10, portion of a voussoir decorated with a palmette.

Le bloc, brisé de tous côtés, ne conserve qu'une faible portion d'une face de parement.

La seule archivolte conservée est ornée d'un bouquet ascendant d'anthémion : cinq feuilles (il en existait une sixième, qui a totalement disparu) aux bords légèrement dentelées et qui se replient encadrent un épais pistil.

Bloc 11 - Claveau (fig. 22)

Dimensions. H.T. : $41 \mathrm{~cm}$; Lg : $43 \mathrm{~cm}$ à l'intrados, $60 \mathrm{~cm}$ cons. au sommet; prof. : $68,5 \mathrm{~cm}$.

Sont conservés l'intrados, les deux faces de joint latérales, complètement lisses et dépourvues du moindre aménagement, et une portion lacunaire d'une archivolte.

L'archivolte porte un rinceau de vigne en relief méplat très peu accusé. On distingue, à droite, une petite portion de la tige principale; son ondulation est meublée de tiges secondaires générant deux feuilles de vigne à cinq folioles, vues de face, et des grappes de raisin. On relève sur les feuilles comme sur le fond des traces de gradine. La partie supérieure du bloc a disparu. 

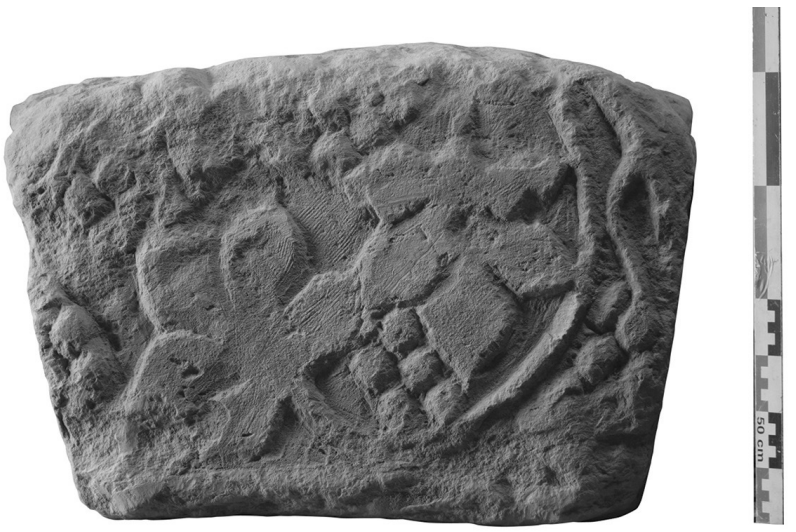

Figure 22 : Bloc 11, fragment d'un claveau orné d'un rinceau de vigne. Cliché Annabelle Cocollos, CG 14.

Figure 22: Block 11, fragment of a voussoir decorated with a vine scroll.

L'intrados nous est demeuré inaccessible mais, selon un croquis ancien, il est orné d'un fleuron de quatre pétales dans un carré sur la pointe, motif qui n'est pas attesté par ailleurs sur le monument. La bordure d'encadrement - une tresse à deux brins ou une torsade - est conservée.

\section{Bloc 12 - Claveau (fig. 23)}

Dimensions : H. cons. : 29,5 cm dont $25 \mathrm{~cm}$ intacts; $\mathrm{Lg}$ max. de l'archivolte : $27,3 \mathrm{~cm}$; prof. à l'intrados : $21 \mathrm{~cm}$, dont $15,5 \mathrm{~cm}$ pour la torsade et ses listels d'encadrement.

Sur la petite partie d'archivolte conservée (fig. 23a) sont visibles, au-dessus d'un listel (H. : 4,3 cm), les vestiges d'un anthémion, à savoir l'extrémité d'un élément de liaison passée dans un bandeau de serrage, et la base d'un bouquet de trois feuilles.

Une faible partie de l'intrados est préservée (fig. 23b) : la bordure est constituée d'une tresse à deux brins sur une moulure convexe bordée par deux listels. D'un caisson n'est conservée qu'une faible portion d'un fleuron, qui n'obéissait pas à la typologie de ceux des blocs 7, 8, 11 et 13 : il comportait peut-être deux corolles de pétales.

\section{Bloc 13 - Claveau (fig. 24)}

Dimensions. H. cons. de l'archivolte : $18 \mathrm{~cm}$; prof. de l'intrados : $55,5 \mathrm{~cm}$, dont $15,5 \mathrm{~cm}$ pour la torsade et ses listels d'encadrement.

Ces deux fragments jointifs conservent une toute petite partie d'une archivolte et surtout son intrados. De l'archivolte est visible l'extrémité de deux feuilles enserrée par un bandeau, ce qui renvoie à un anthémion. À l'intrados, on observe un fleuron de quatre pétales dentelés, posés sur la diagonale, fleuron bordé par une tresse à deux brins sur une moulure légèrement convexe encadrée par deux listels.
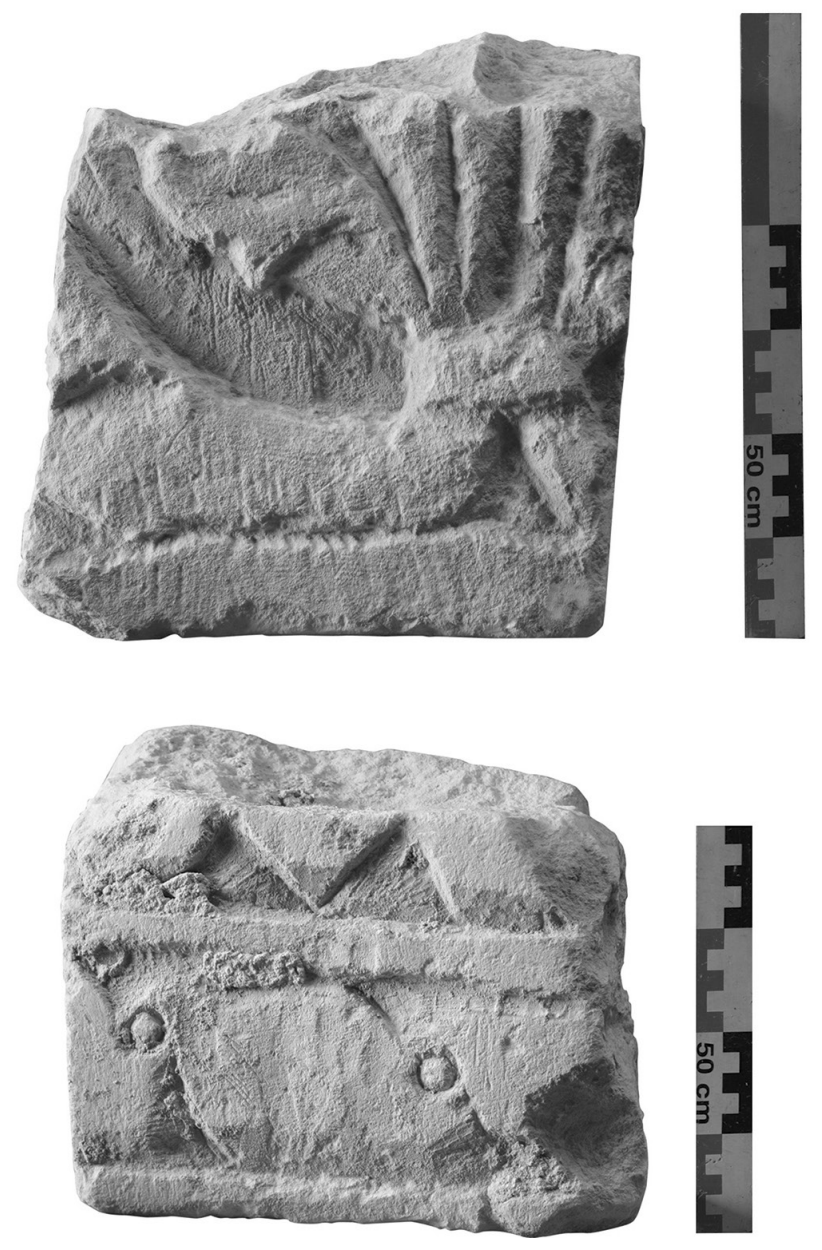

Figure 23 : Bloc 12. (a) Fragment d'un claveau orné d'un anthémion. (b) Intrados. Clichés Annabelle Cocollos, CG 14.

Figure 23: Block 12. (a) Fragment of a voussoir decorated with an anthemion. (b) Intrados.

\section{Bloc 14 - Claveau (fig. 25)}

Dimensions. H.T. : $96 \mathrm{~cm}$; prof. à l'intrados : $69,5 \mathrm{~cm}$; Lg des archivoltes : $41,5 \mathrm{~cm}$ et $54 \mathrm{~cm}$. Sur la face au griffon, Lg de la face de joint droite : $80,8 \mathrm{~cm}$; $\mathrm{Lg}$ de la face oblique qui la recoupe : $25 \mathrm{~cm}$.

La face supérieure, parallèle à l'intrados, constitue le lit d'attente, sans doute retaillé. Les deux faces de joint latérales sont lisses et dépourvues de trou de bardage et de scellement.

Sur la première archivolte $(\mathrm{H} .52 \mathrm{~cm})$ est conservée la partie postérieure d'un griffon bondissant vers la gauche, soit une petite partie de l'abdomen, la patte postérieure gauche, dont la cuisse est légèrement galbée, et la longue queue, dont le départ et l'extrémité sont visibles; l'extrémité d'une aile est conservée, qui assure l'identification du sujet (fig. 25a). Le couronnement $(\mathrm{H} .: 16 \mathrm{~cm})$, sur lequel le bandeau sculpté s'amortit par un congé, est constitué par un bandeau (H. : 


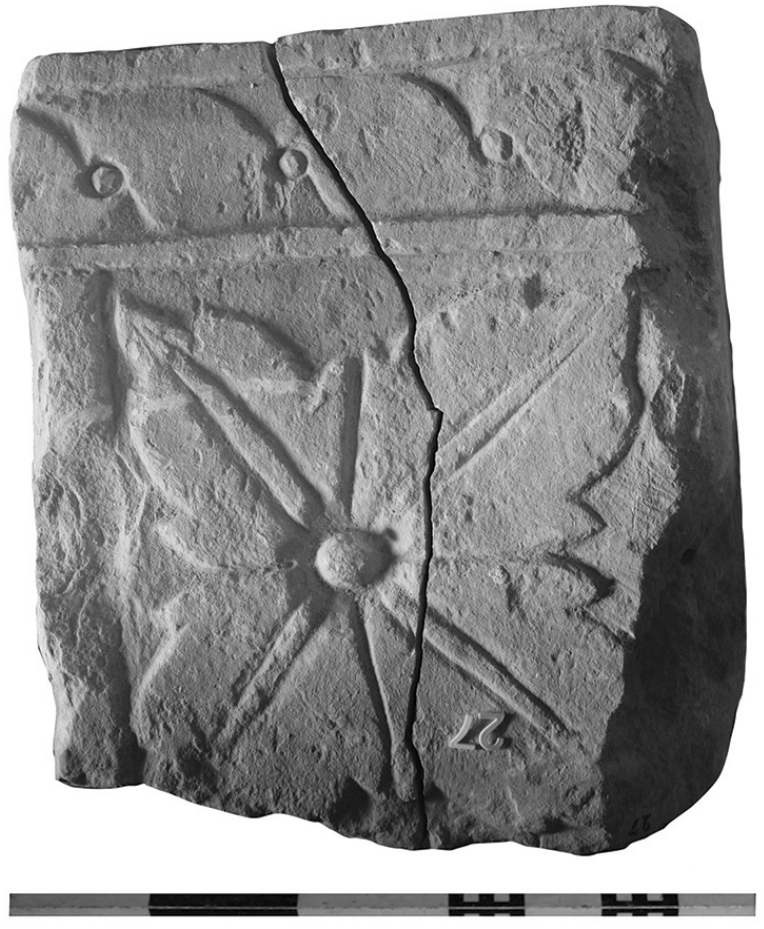

Figure 24 : Bloc 13, intrados. Cliché Annabelle Cocollos, CG 14. Figure 24: Block 13, intrados.

$9,5 \mathrm{~cm}$ ) sculpté d'une tresse à deux brins avec bouton, encadrée par deux listels.

La seconde archivolte (H. : 55,5 cm) est ornée d'une portion de rinceau acanthisé (fig. 25b). Une longue bractée, vue de profil, livre passage à deux bractées, elles aussi vues de profil : l'une constitue la tige principale du rinceau, l'autre une tige secondaire; l'écoinçon est meublé de ce qui semble être un caulicole à gaine lisse et collerette végétalisée, engendrant un calice complètement écrasé sous le couronnement. Des portions de ce qui semble être des bractées de tiges secondaires meublant les enroulements du rinceau sont visibles de part et d'autre de ces éléments centraux. Le couronnement (H. : 18,1 cm) est composé d'un bandeau $(12,6 \mathrm{~cm})$ sculpté de trois feuilles inclinées vers la gauche, encadrées par deux listels; elles présentent une nervure axiale, sont légèrement dentelées et leur extrémité se replie sur le limbe. Au-dessus de chaque archivolte figure une plage lisse.

L’intrados (fig. 25c) porte un décor végétal peuplé de motifs figurés $(\mathrm{H}$. de la scène $=\mathrm{Lg}$ du bloc au soffite : $40,2 \mathrm{~cm}$ ). De ce qui semble être une tige axiale s'échappent symétriquement deux tiges secondaires incurvées dessinant un motif lyriforme. La tige de droite est la mieux conservée : sa partie supérieure est recouverte d'une feuille et un masque (H. : $22 \mathrm{~cm}$, dont $15,5 \mathrm{~cm}$ pour le visage) y est accroché par un bandeau. Ce masque est tourné vers la gauche; les traits du visage sont très accusés, le menton proéminent, la bouche ouverte présente une lèvre inférieure épaisse, l'œil est enfoncé dans son orbite; le masque est coiffé d'un couvrechef qui laisse visible la partie inférieure de la chevelure. La partie gauche de la composition est largement bûchée, mais laisse deviner un masque identique tourné lui aussi vers la gauche.

\section{Bloc 15 - Claveau (fig. 5)}

Le bloc, découvert en 1828 (ou 1829?), n'est connu que par des dessins de Charles-Édouard Lambert. Imprécis, ils assurent cependant que ce bloc était jointif du précédent. Les trois faces ornées étaient conservées, et un des joints montants était creusé d'un trou de louve, assurément lié au remploi.

Une des archivoltes porte une grande partie d'un griffon dont la queue relevée est liée à celle du griffon du bloc 14; les deux pattes postérieures, le corps et une aile sont conservés; selon le dessin, un sexe masculin aurait été très nettement indiqué. L'archivolte était couronnée par une tresse sur un bandeau.

Sur l'autre archivolte est dessinée une portion de rinceau : le dessinateur n'a pas bien compris le décor, mais il n'est pas douteux que celui-ci reproduit le schéma du bloc 14, à savoir une ample bractée donnant naissance à deux feuilles plus petites, séparées par une tige; le dessin livre cependant une information supplémentaire, puisqu'il figure, à droite, une bractée secondaire et un enroulement.

Le dessin de l'intrados confirme la structure symétrique d'une composition qui, superposant des bouquets encadrés par de longues tiges, doit être interprétée comme un candélabre végétal.

\section{Bloc 16 - Claveau (fig. 26)}

Dimensions. H.T. : $66,5 \mathrm{~cm}$; H. couronnement : $18 \mathrm{~cm}$; Lg max. de l'archivolte : $67 \mathrm{~cm}$; prof. à l'intrados : $68 \mathrm{~cm}$.

Le lit d'attente est creusé d'un trou de louve (fig. 26a). La face de joint montant droite, parallèle à l'intrados, porte un défoncé encadré par deux bandeaux d'anathyrose; le bloc appartenait donc au départ, vertical, d'une archivolte, ce que confirme le décor de l'intrados.

Une archivolte est bien conservée (fig. 26b) : elle porte un anneau épais, bordé par un listel et une baguette, dans lequel est passé un bâton à l'extrémité recourbée, identifiable à un pedum. Le couronnement est constitué d'une tresse à deux brins sur un bandeau bordé par deux listels.

La partie gauche de l'autre archivolte (fig. 26c) est lisse, apparemment laissée au stade de l'épannelage; à droite, on distingue une bractée.

Sur l'intrados (fig. 26c), on identifie avec difficulté les vestiges d'un bouquet bordé de chaque côté par un calice à 

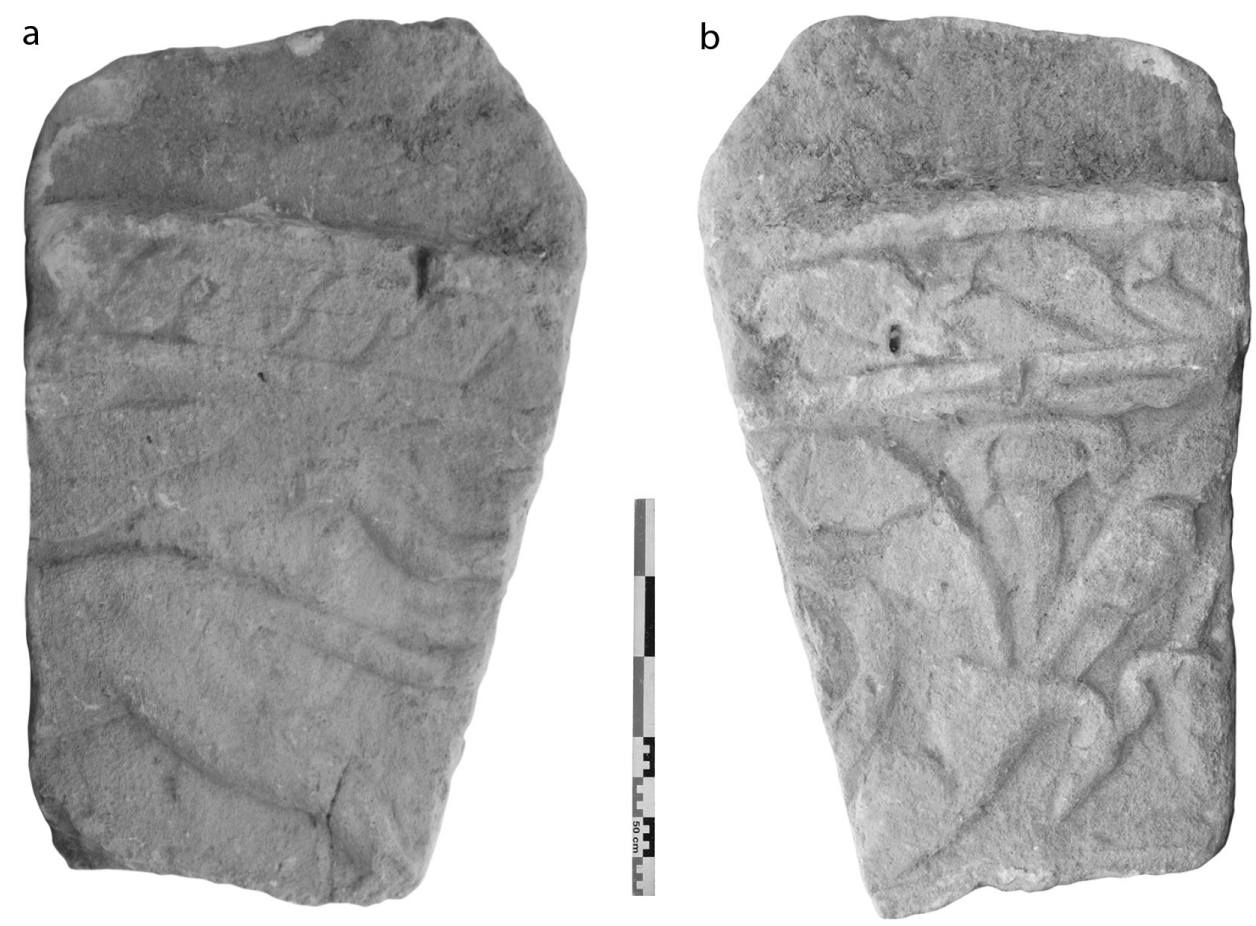

Figure 25 : Bloc 14. (a) Archivolte ornée d'un griffon et couronnée par une tresse. (b) Archivolte ornée d'un rinceau et couronnée par un rang de feuilles. (c) Intrados décoré d'un candélabre végétal auquel sont suspendues des têtes. Clichés Annabelle Cocollos, CG 14.

Figure 25: Block 14. (a) Archivolt decorated with a griffin and crowned by a plait. (b) Archivolt decorated with a scroll and crowned by a row of leaves. (c) Intrados side decorated with a vegetal candelabrum from which heads are suspended.

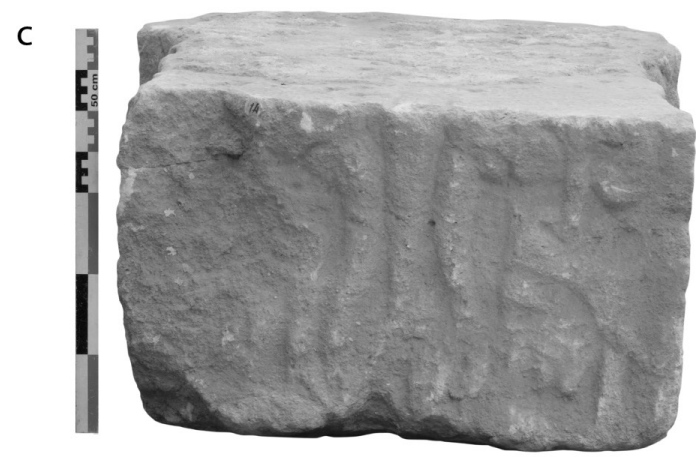

deux brins ouvrant sur un pistil lancéolé; il s'agit vraisemblablement du culot d'un candélabre végétal.

Bloc 17-Claveau (fig. 27)

Dimensions. H.T. : $86 \mathrm{~cm}$; Lg : $57,5 \mathrm{~cm}$; prof. à l'intrados : $70 \mathrm{~cm}$; prof. au lit d'attente : $70 \mathrm{~cm}$.

Les deux faces de joint latérales sont conservées; le lit d'attente ne porte aucun aménagement.

Les deux archivoltes sont en bon état. Sur l'une est conservée une portion d'un anthémion (fig. 27a) : un bouquet dressé dont la base est enserrée par un bandeau d'où émergent les deux volutes terminales des éléments de liaison; le bouquet lui-même est constitué de deux feuilles dentelées vues de profil, à trois grosses folioles, affrontées symétriquement de part et d'autre d'un calice à deux brins. Larchivolte est couronnée par un anthémion sculpté sur un talon, lui-même couronné par un listel $(\mathrm{H} .: 17,5 \mathrm{~cm})$ : la composition est identique à celle qui orne le couronnement des blocs 7, 8 et 9. Au-dessus du couronnement apparaît une plage lisse.

Lautre archivolte développe un décor différent (fig. 27b) : au-dessus d'un listel $(\mathrm{H} .: 5 \mathrm{~cm})$ sont préservées deux bractées affrontées, vues de profil et liées par un petit bandeau de serrage; la bractée droite jaillit d'un élément qui semble être un caulicole. Les deux bractées sont composées de larges folioles dentelées, au limbe faiblement animé. On aurait pu penser à un rinceau courant vers la gauche, mais, aucune tige ne jaillissant de la bractée de droite dont l'extrémité est conservée, l'hypothèse doit être abandonnée. Nous sommes peut-être en présence de deux rinceaux, dans le cadre soit d'une composition juxtaposant des rinceaux très courts - par exemple dans une composition de $S$ couchés comme celle qui apparaît sur une archivolte de Saintes (fig. 28) - soit de deux rinceaux jaillissant de culots situés à chacune des 

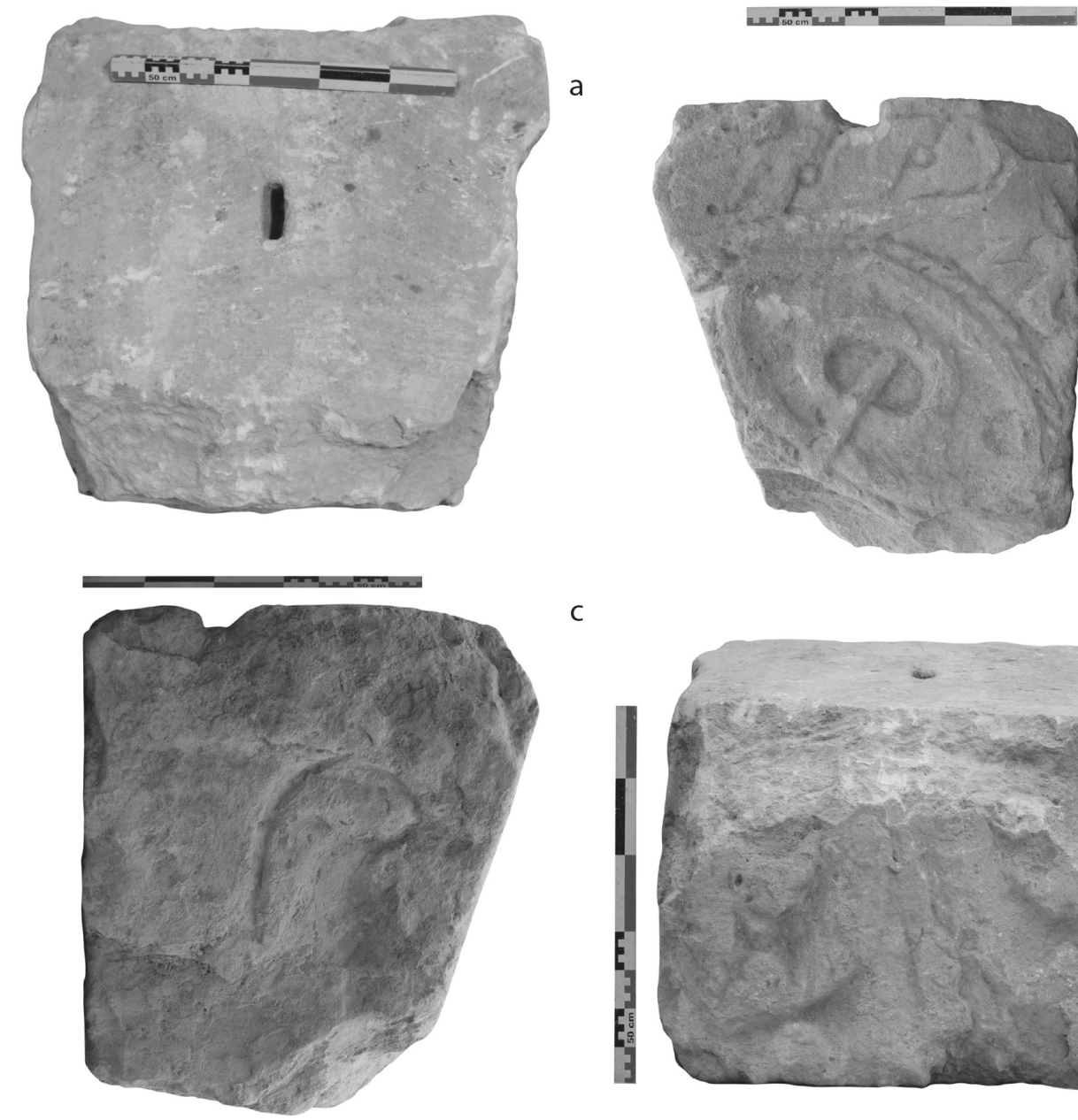

c

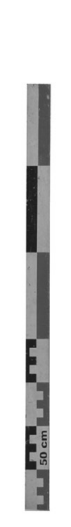

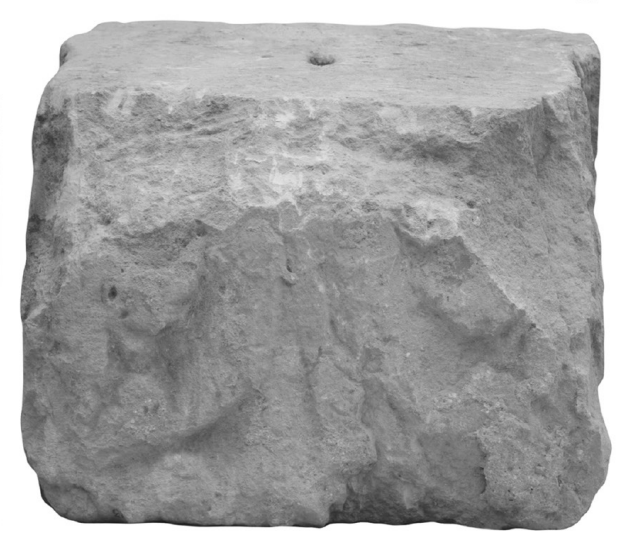

b

Figure 26 : Bloc 16. (a). Lit d'attente. (b) Archivolte ornée d'un pedum passé dans un anneau et couronnée par une tresse. (c) Archivolte portant les vestiges d'un rinceau. (d). Intrados conservant les vestiges d'un candélabre végétal. Clichés Annabelle Cocollos, CG 14.

Figure 26: Block 16. (a). bedding surface. (b) Archivolt decorated with a pedum in a ring and crowned by a guilloche. (c) Archivolt with traces of a scroll. (d). Intrados with preserved fragments of a vegetal candelabrum. extrémités de l'archivolte. Le couronnement est plus important que sur l'autre face $(\mathrm{H} .: 23,8 \mathrm{~cm})$ : sur un talon très sommaire figure une file de feuilles d'acanthe alternant avec des joncs; les feuilles présentent une côte axiale plate, s'élargissant très légèrement vers le bas, autour de laquelle sont disposées cinq folioles lisses, la foliole axiale se retournant vers la droite, comme animée par le vent; les joncs intermédiaires, largement masqués, présentent une nervure axiale et se retournent eux aussi au sommet.

L'intrados (fig. 27c) est orné d'un personnage incomplètement conservé, de la partie inférieure des jambes au cou; de sexe masculin, il est potelé et l'identification d'un Amour ne fait pas de difficulté. Sa jambe gauche est pliée, sa jambe droite allongée vers l'arrière; le torse est affecté d'une forte torsion et vu presque de face; le bras gauche est bûché, le bras droit tendu, écarté du corps. L'Amour, figuré comme un jeune enfant, tient un objet dans chaque main : dans la main gauche, un disque identifiable à un tambourin, dans la main droite un objet allongé, peut-être une baguette. Cet objet est partiellement masqué par une forme curviligne fermée que nous ne parvenons pas à identifier, pas plus que nous ne comprenons les deux masses (des tentures?) qui tombent symétriquement, à droite et à gauche, dans la partie inférieure du relief, le long des bandeaux d'encadrement.

\section{Bloc 18 - Claveau (fig. 29)}

Dimensions. H.T. : $68 \mathrm{~cm}$; Lg max. : $40 \mathrm{~cm}$; prof. cons. au couronnement : $34 \mathrm{~cm}$.

Ces deux fragments jointifs d'un claveau ne conservent qu'une partie du décor d'archivolte et son couronnement. Le bandeau (H. cons. : $31 \mathrm{~cm})$ était sculpté d'un anthémion dont est préservée une partie d'un bouquet dressé (deux feuilles et un pistil lancéolé). Le couronnement (H. : $19 \mathrm{~cm})$ est un épais quart-de-rond habillé d'un motif très sommaire et couronné d'un listel : entre deux feuilles apparaît un calice lisse à peine esquissé; on a là une version très simplifiée, sans doute simplement épannelée, de l'anthémion qui apparaît sur plusieurs blocs en pareille position. Au-dessus du couronnement se trouve une zone lisse. 
a

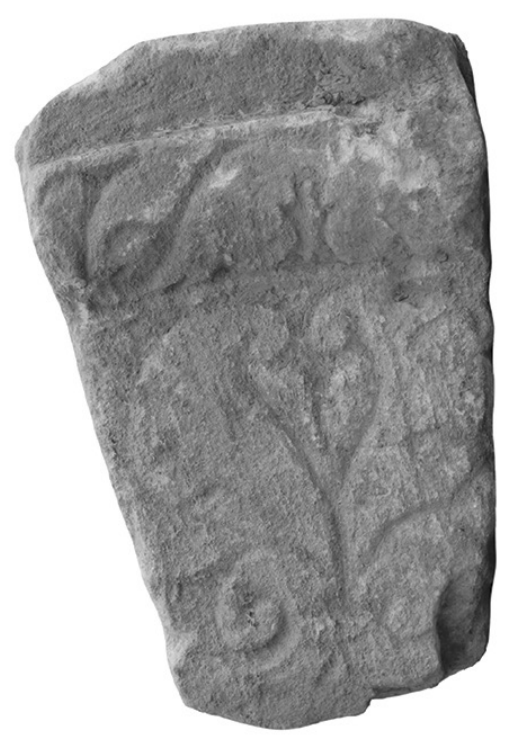

b

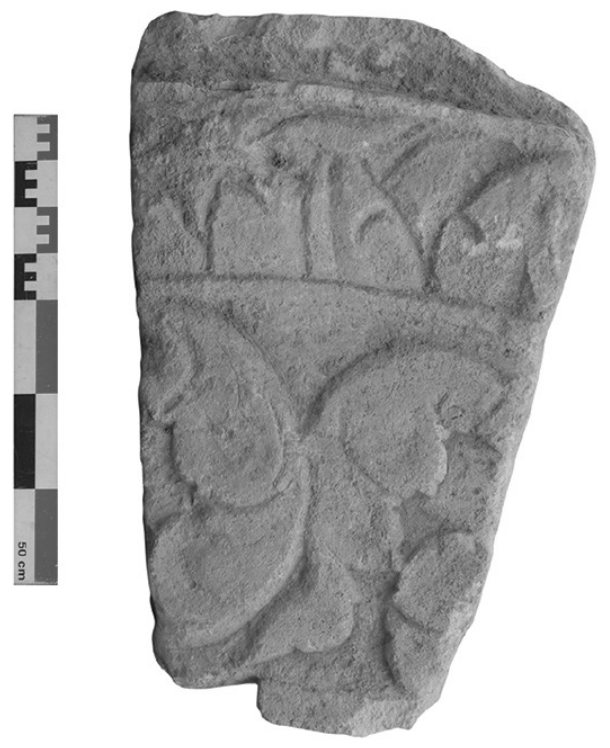

c

Figure 27 : Bloc 17. (a) Archivolte ornée d'un anthémion et couronnée par un anthémion. (b) Archivolte portant la jonction de deux rinceaux, couronnée par un rang de feuilles et joncs. (c) Intrados orné d'un Amour. Clichés Annabelle Cocollos, CG 14.

Figure 27: Block 17. (a) Archivolt decorated with an anthemion and crowned by a palmette (b) Archivolt with the junction of two scrolls, crowned by a row of leaves and rush (c) Lower side decorated by a cupid.

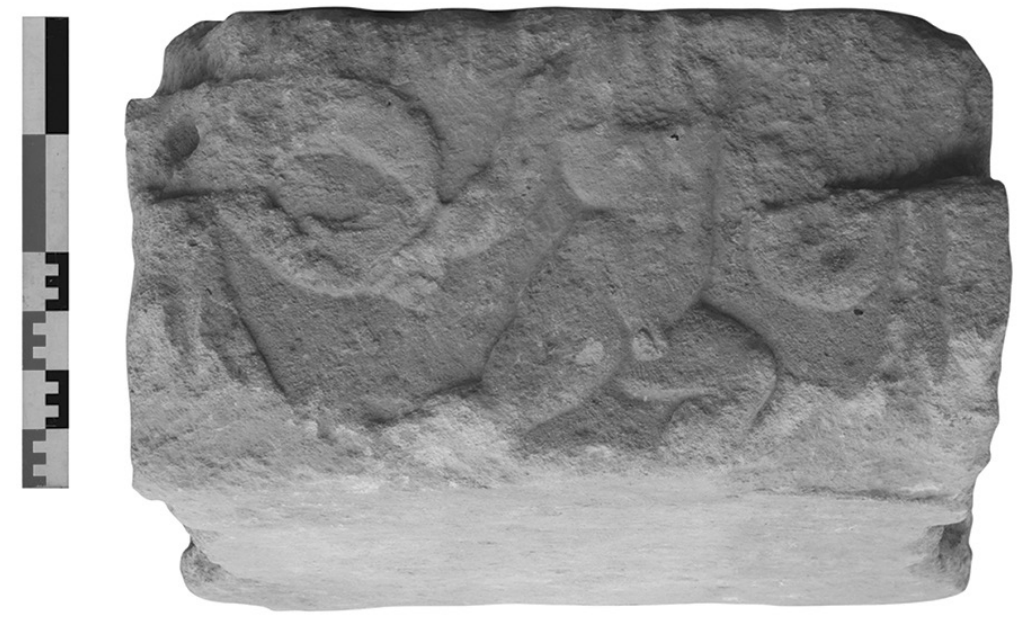

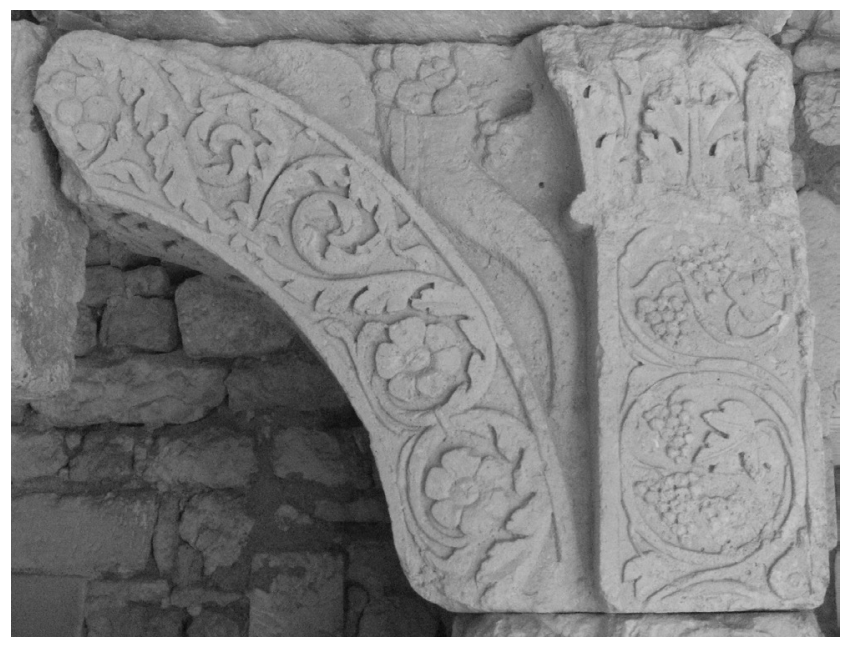

Figure 28 : Vestiges d'un monument funéraire à décor dionysiaque de Saintes. L'archivolte est ornée de courts rinceaux dont un claveau de Bayeux (Figure 27b) pourrait porter une variante. Cliché Grégory Schütz.

Figure 28: Remains of a funerary monument in Saintes with a dionysiac decor. The archivolt is decorated with short scrolls of which a Bayeux voussoir (Figure 27b) could be a variation. 


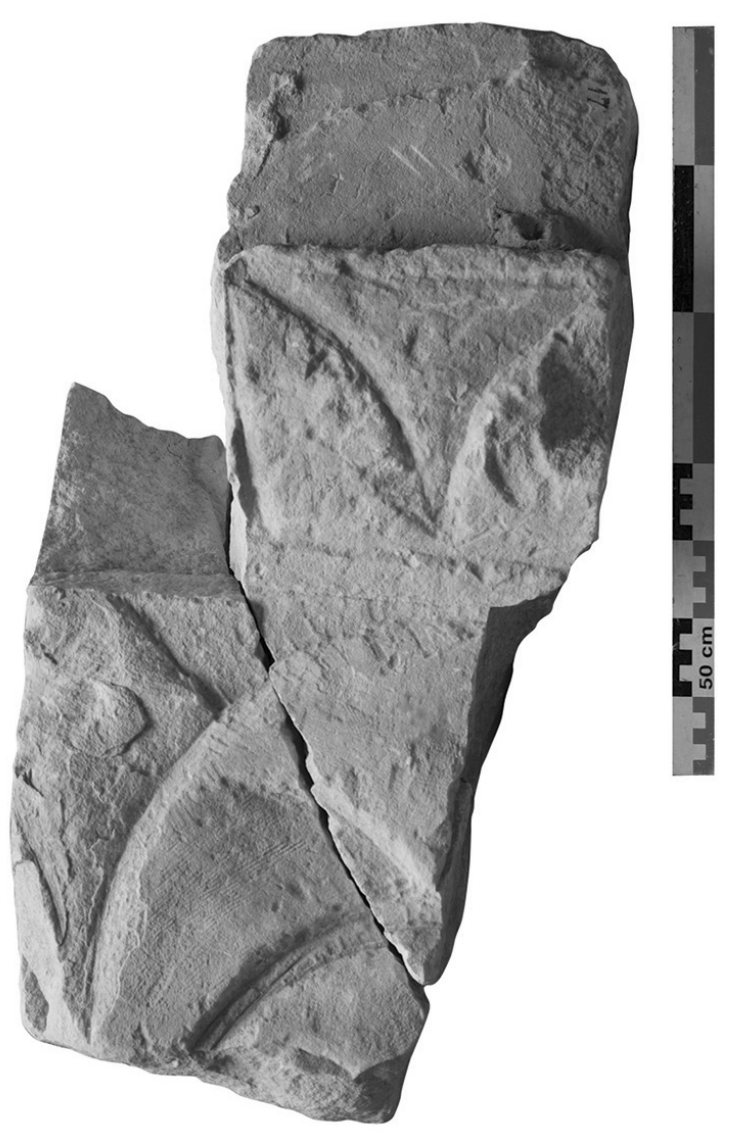

Figure 29 : Bloc 18, archivolte portant les vestiges d'un anthémion et couronnée par un anthémion épannelé. Cliché Annabelle Cocollos, CG 14.

Figure 29: Block 18, archivolt decorated with an anthemion and crowned by a partially carved anthemion.

\section{Bloc 19-Fragment du couronnement} d'une archivolte (fig. 30)

Dimensions. H.T. : 22,6 cm; $\mathrm{Lg}$ : $54,5 \mathrm{~cm}$ (dont $40,5 \mathrm{~cm}$ de lg pour le décor); prof. : $28 \mathrm{~cm}$.

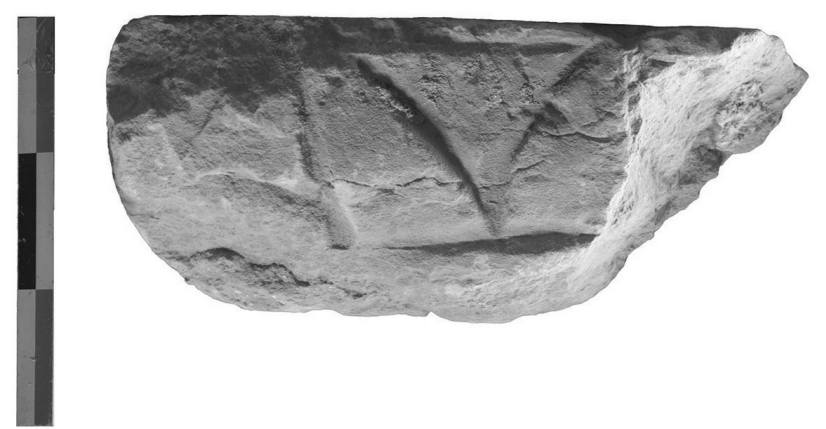

Figure 30 : Bloc 19, couronnement d'une archivolte. Cliché Annabelle Cocollos, CG 14.

Figure 30: Block 19, the crowning of an archivolt.
Le fragment ne conserve que la moulure de couronnement, identique à celle du bloc 18 : une vague composition végétale est sculptée sur un quart-de-rond.

\section{Type IV/ Bloc appartenant à la partie supérieure du monument}

Bloc 20 - Bloc d'assise (fig. 31)

Dimensions. H.T. : $94 \mathrm{~cm}$; Lg cons. : $92 \mathrm{~cm}$; prof. cons. : $61,5 \mathrm{~cm}$.

Le lit de pose a fait l'objet d'une retaille et seule une faible portion en est conservée. Le bloc est cassé à gauche et à
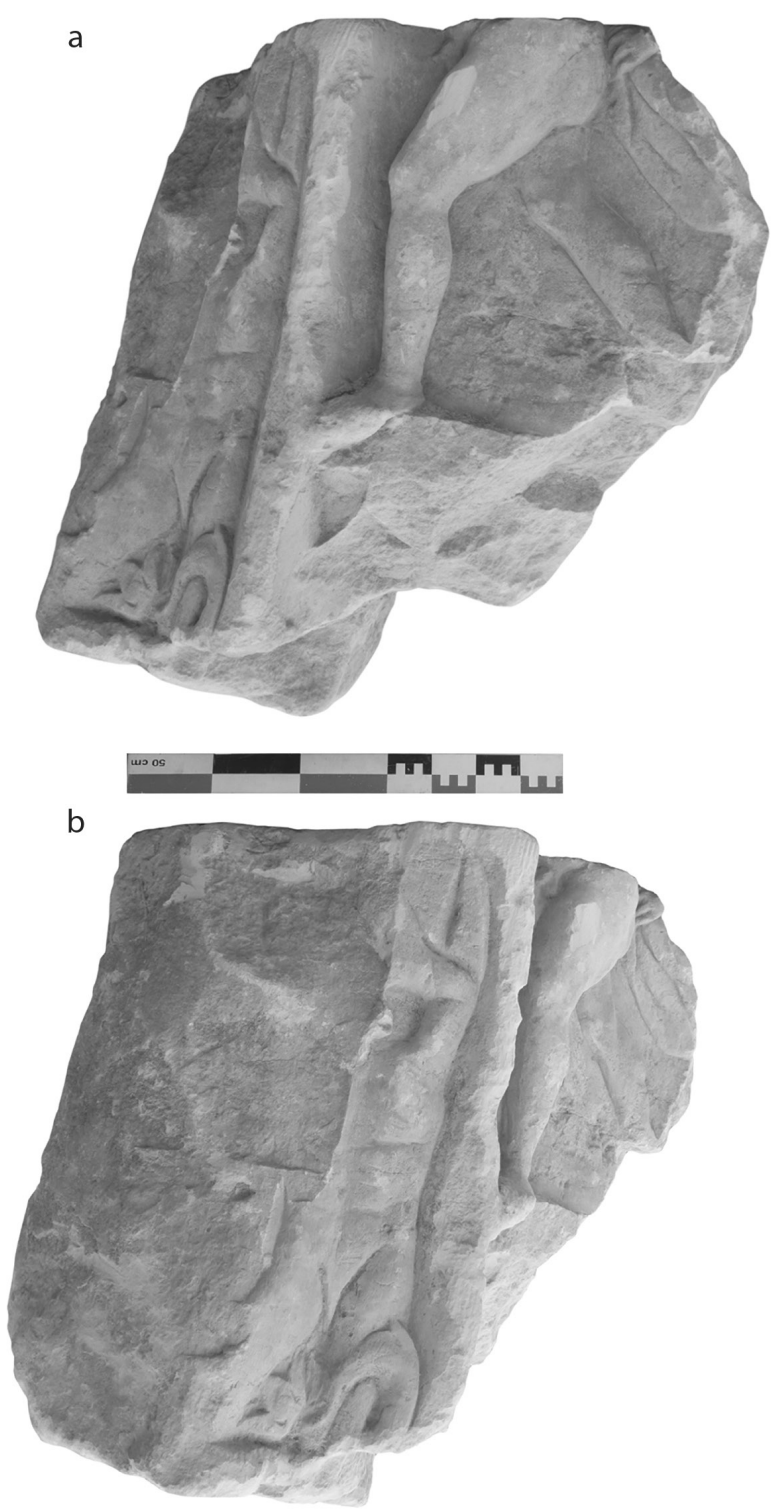

Figure 31 : Bloc 20. (a) Vue d'ensemble de la face ornée. (b) Le triton (clichés Annabelle Cocollos, CG 14).

Figure 31: Block 20. (a) View of the decorated face. (b) The triton. 
droite. Le lit d'attente est mieux conservé : lisse, il est creusé d'un trou de louve $(2,6 \times 10,4 \mathrm{x}-13,1 \mathrm{~cm})$. Les deux lits de pose et d'attente ne sont pas parallèles, ce dernier étant déversé vers l'intérieur. La face arrière, assez bien conservée, est lisse.

Le bloc porte deux reliefs séparés par une moulure légèrement convexe dont le tracé est curviligne, ce qui rend difficile la localisation du bloc dans le monument (infra, $\$$ IV). Le panneau de gauche (H. : $83 \mathrm{~cm}$; Lg cons. : $24,5 \mathrm{~cm}$ ) est cantonné en bas et en haut par un bandeau lisse (fig. 31b). Du relief, on ne peut identifier que la partie droite; on reconnait cependant un personnage hybride dont la jambe gauche s'achève par une nageoire trifide. Le bassin ne présente aucun organe génital, mais le torse semble bien masculin; le bras gauche levé et replié au-dessus de la tête devait tenir un attribut - conque ou aviron - non identifiable. La nageoire assure l'identification et permet d'écarter l'hypothèse d'un géant anguipède : c'est Triton lui-même ou un triton qui est ici figuré ${ }^{17}$.

La partie droite du bloc (fig. 31a) porte, au-dessus d'un bandeau abattu, un panneau sculpté (H. cons. : $51 \mathrm{~cm}$; Lg cons., qui doit être presque complète : $47 \mathrm{~cm}$ ) dont ne subsiste que la partie inférieure, représentant sur un fond lisse un homme, jambe et bassin nus, marchant à grande enjambée vers la gauche : la jambe gauche, au modelé vigoureux, se détache nettement du fond $(6 \mathrm{~cm})$, tandis que la jambe droite est traitée en bas-relief; la représentation anatomique est soignée et les masses musculaires bien indiquées. Le pied gauche est étrangement tourné vers l'extérieur. Les organes génitaux sont bien visibles, qui ôtent tout doute sur le genre du personnage; sa main gauche, conservée sur le fond du relief, tient un objet indéterminé (ou une draperie?). Ce panneau, qui en surmontait un autre dont rien n'est conservé, est pratiquement complet en longueur, puisque le fond du relief se relève nettement à droite, signalant la proximité de la bordure.

Les blocs qui nous sont parvenus proviennent des assises médianes du monument : rien ne nous est conservé de ses parties basses, rien de son entablement ni de son probable attique. Cette petite collection nous donne cependant accès à un certain nombre d'informations, qui touchent à la datation du monument, à sa typologie et à la thématique de son décor figuré. Nous affronterons d'abord le problème chronologique, qui aura son importance au moment de la constitution du dossier comparatif.

17. Pour leur iconographie, voir Icard-Gianolio 1997a et b; pour la difficulté de distinguer le dieu des tritons génériques, voir Icard-Gianolio 1997 b, p. 83.

\section{UN MONUMENT SÉVÉRIEN}

La datation de cet ensemble ne pose guère de problème : les blocs fournissent des informations absolument convergentes, ce qui renforce évidemment leur rapprochement (fig. 32).

Les files de feuilles inclinées qui couronnent certaines archivoltes (blocs 14 et 17 : fig. 32a et b) renvoient à une datation aux II elIII $^{\mathrm{e}}$ siècles, mais c'est surtout à partir du dernier quart $\mathrm{du} \mathrm{II}^{\mathrm{e}}$ siècle que ces motifs qui semblent animés par le vent se répandent sur les modénatures de Gaule romaine : si les séries lapidaires de Gaule de l'Est et du Nord-Est en fournissent de très nombreux exemples ${ }^{18}$, ces variantes sont reprises en Aquitaine (Tardy, 2005, fig. 90) et Lyonnaise ${ }^{19}$. La typologie des anthémions (blocs 7 et 9 : fig. 32c et d) confirme une datation postérieure au milieu du II $^{\mathrm{e}}$ siècle : la composition a subi une simplification structurelle très bien documentée; alors qu'ils étaient auparavant constitués de bouquets alternativement dressés et tombants reliés par des feuilles ou des tiges, les anthémions sont dorénavant fréquemment formés d'une suite de motifs végétaux ressemblant à des rais de cœur et dont les extrémités liées par un bandeau de serrage tiennent lieu de bouquets ascendants ${ }^{20}$. Le découpage et le modelé des folioles sur les rinceaux $B$ et $C$ du bloc 1 (fig. 32e et $\mathrm{f}$ ) renforcent ces conclusions : les digitations courtes et épaisses comme le creusement du limbe en cuiller s'inscrivent dans des séries très étoffées. Des rinceaux très voisins de notre type $\mathrm{B}$ ornent les pilastres d'une stèle de Lillebonne (Espérandieu, IV, 3097; fig. 33); d'autres apparaissent sur la Porte de Mars, à Reims (fig. 34). Mais ces feuilles ne sont pas l'apanage des rinceaux : pour nous en tenir aux parallèles les plus proches sur le plan géographique, nous citerons les chapiteaux du baldaquin tétrastyle de la place de la Fontaine, à Rouen (Follain et al., 1996, cliché p. 11) ou des claveaux de Genainville (Mitard, 1993, p. 165). Les vestiges de colonne engagée (blocs 5 et 6) confortent cette orientation chronologique : les bractées du rinceau à tiges parallèles, et surtout les folioles de la collerette qui surmonte le personnage masculin (fig. $32 \mathrm{~g}$ ) présentent un découpage et un modelé qui trouvent leur équivalent sur de très nombreux blocs de Périgueux à partir du milieu du II siècle (Tardy, 2005, p. 124 et 126); en contexte régional, un parallèle est proposé par un rinceau de Jublains (Maligorne, 2006, fig. 104 et p. 127). Particulièrement caractéristiques sont le modelé convexe du limbe et la façon dont il se plisse pour se relever près du bord.

18. Numrich 1997, pl. 4.3, 28.1, 3 et 6, 30.4, 36.1; Mitard 1993, p. $134, \mathrm{n}^{\circ} 19 ;$ p. $143, \mathrm{n}^{\circ} 63$.

19. Pour un parallèle presque exact de la file de feuilles d'acanthe et de joncs du bloc 17, voir Maligorne 2006, fig. 106.

20. Tardy 1994, type D des anthémions, p. 150 et fig. 71. De nombreux exemples à Genainville : Mitard 1993, p. $130, \mathrm{n}^{\circ} 5$, p. $131, \mathrm{n}^{\mathrm{os}} 11,12$, p. $134, n^{\text {os }} 19-22$, p. $143, n^{\text {os }} 60,61,64,65$. pl. $17-1$. 
a

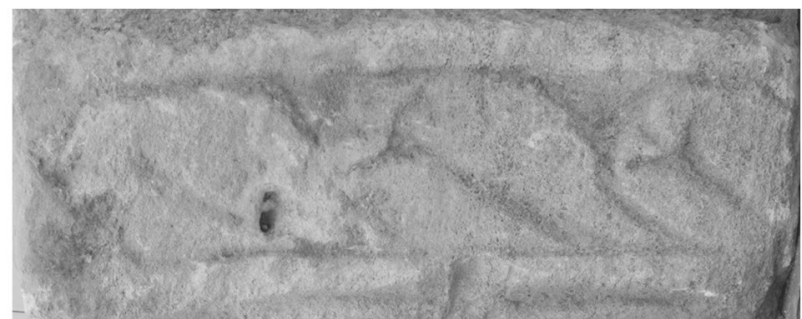

C

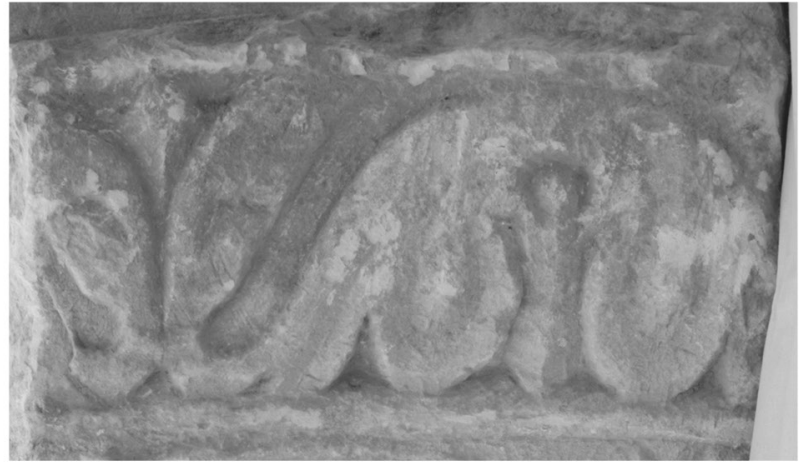

f

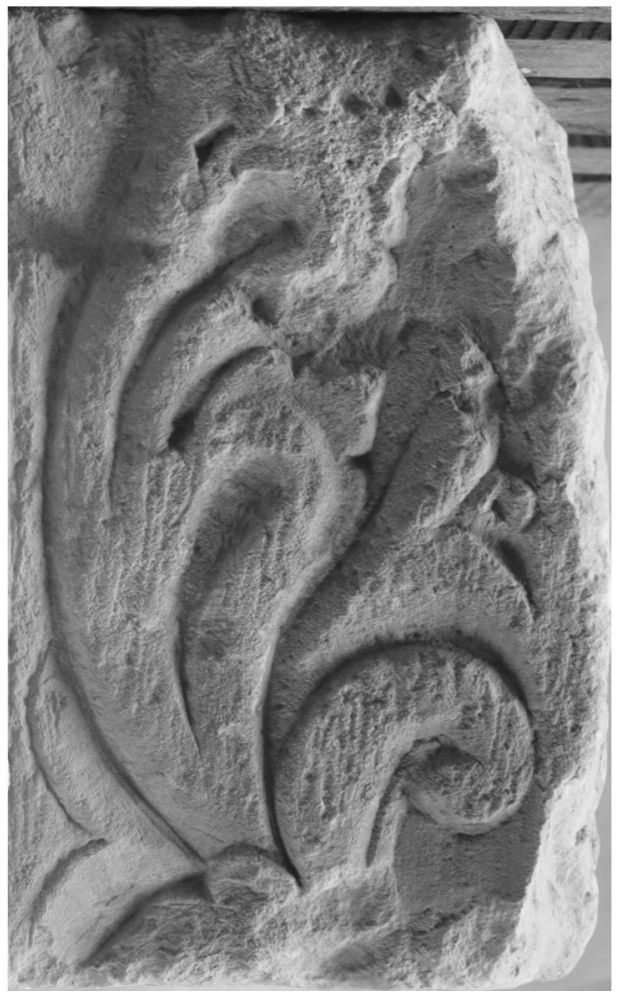

b

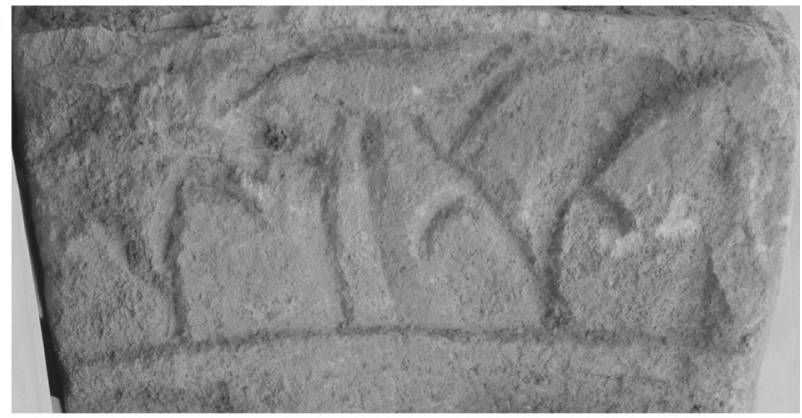

d

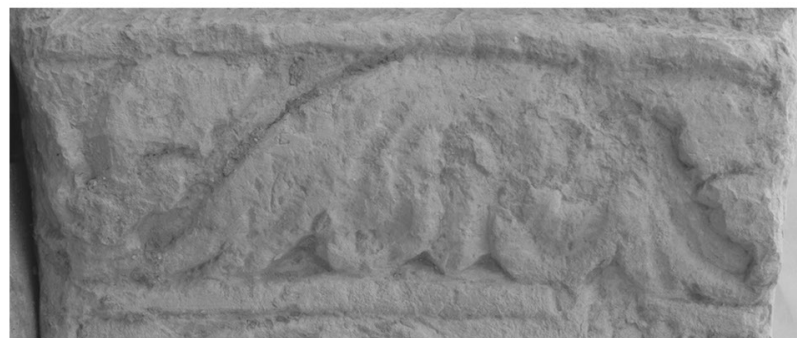

e

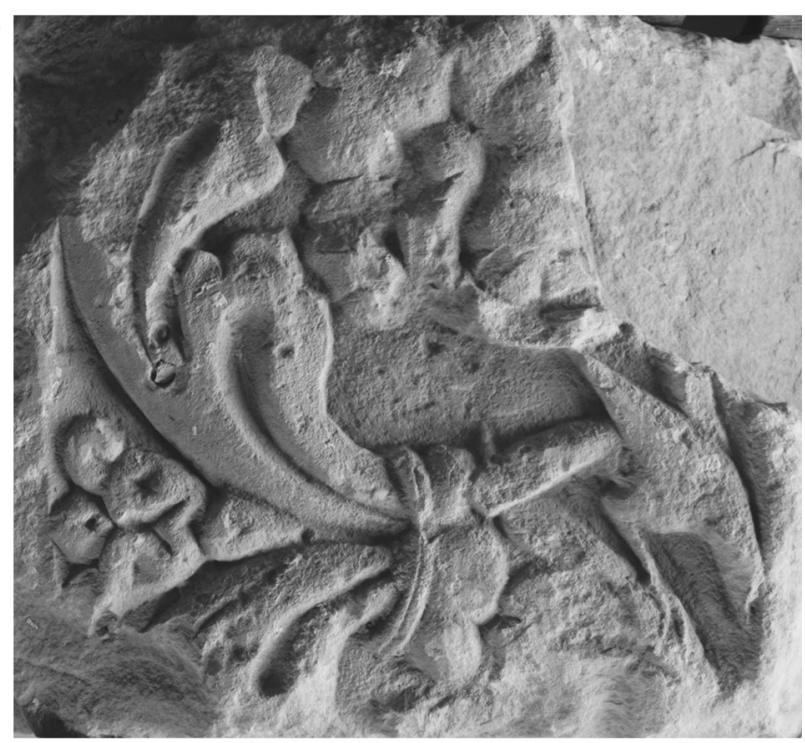

g

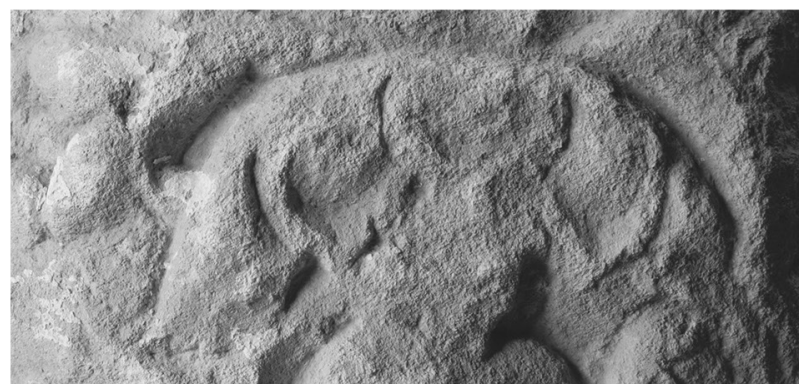

Figure 32 : Le décor architectural non figuré. (a) Motifs de feuilles inclinées du bloc 14. (b) Composition de feuilles inclinées et joncs du bloc 17. (c, d) Anthémions des blocs 79. (e et f) Rinceaux du bloc 1. (g) Collerette de caulicole sur le candélabre végétal du bloc 6. Clichés Annabelle Cocollos, CG 14.

Figure 32: The non-figured architectural decor. (a) Inclined leaf motif of block 14. (b) Row of inclined acanthus and water plant leaves from block 17. (c, d) Palmettes from block 79. (e andf) Scrolls from block 1. (g) Caulicole collar on vegetal cadelabrum from block 6. 


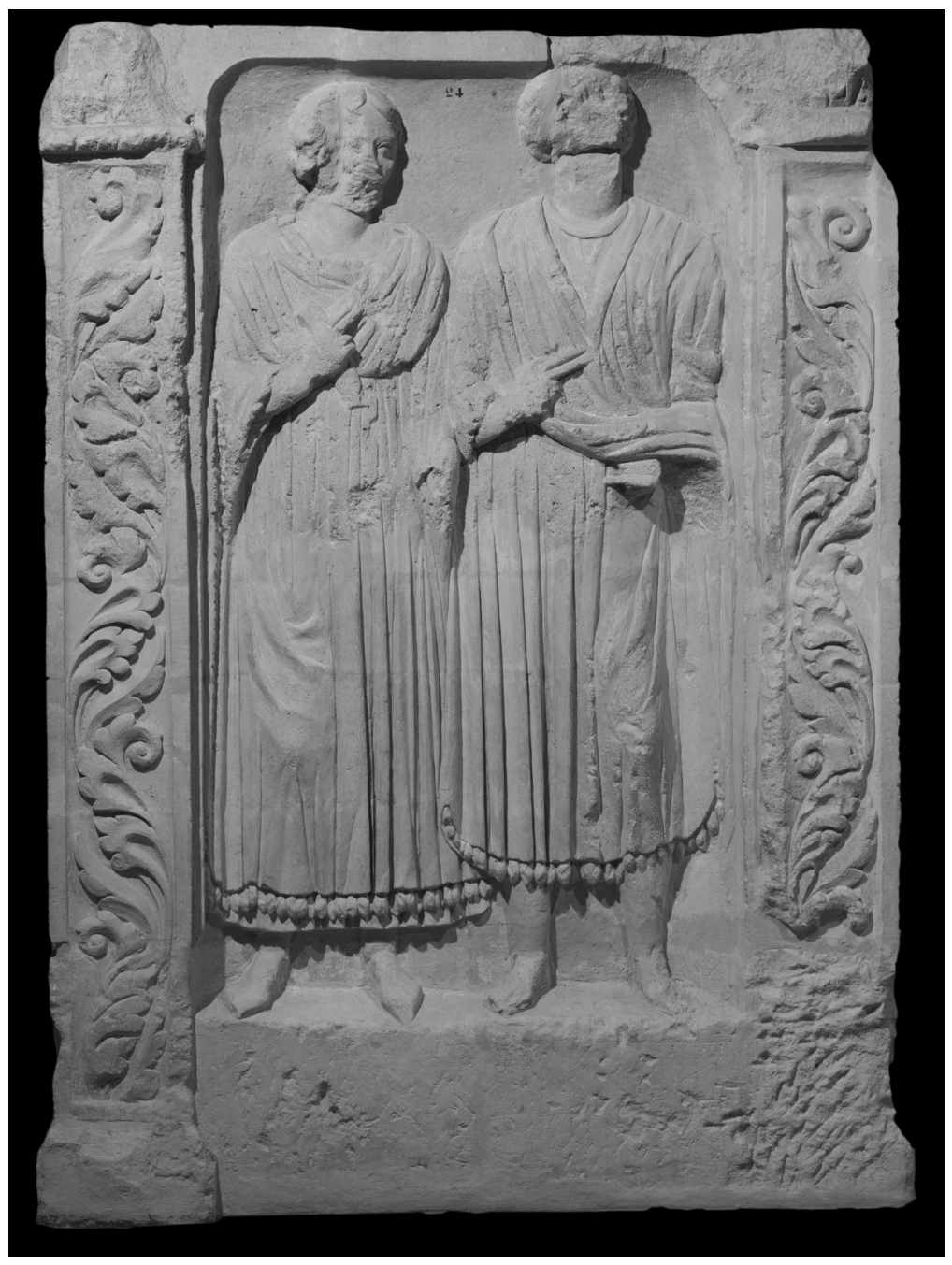

Figure 33 : Stèle funéraire découverte à Lillebonne et exposée au musée des antiquités de Rouen, inv. 195.2 (A). C CG 76, musée département des antiquités Rouen, cliché Yohann Deslandes.

Figure 33: Funerary stele discovered at Lillebonne and exhibited in the Antiquities Museum in Rouen, inv. 195.2 (A).

Si nous avions dû nous en tenir à ces observations, il aurait fallu défendre une datation assez large, dans la deuxième moitié du II ${ }^{\mathrm{e}}$ siècle et le premier tiers du $\mathrm{III}^{\mathrm{e}}$ siècle, ce qui en soi n'aurait pas été un résultat négligeable. Cependant, c'est l'enquête comparative régionale qui fournit les résultats les plus satisfaisants, en nous permettant non seulement de préciser cette datation, mais encore d'identifier l'intervention d'une officine de tailleurs de pierre active dans la région.

Des rinceaux de vigne ornent en Gaule de très nombreux supports, colonnes ou pilastres ${ }^{21}$. Ils ont généralement un caractère générique, qui n'en facilite pas la datation. Les

21. Pour un premier inventaire, très incomplet mais encore très utile, voir Walter 1975, p. 45-56.

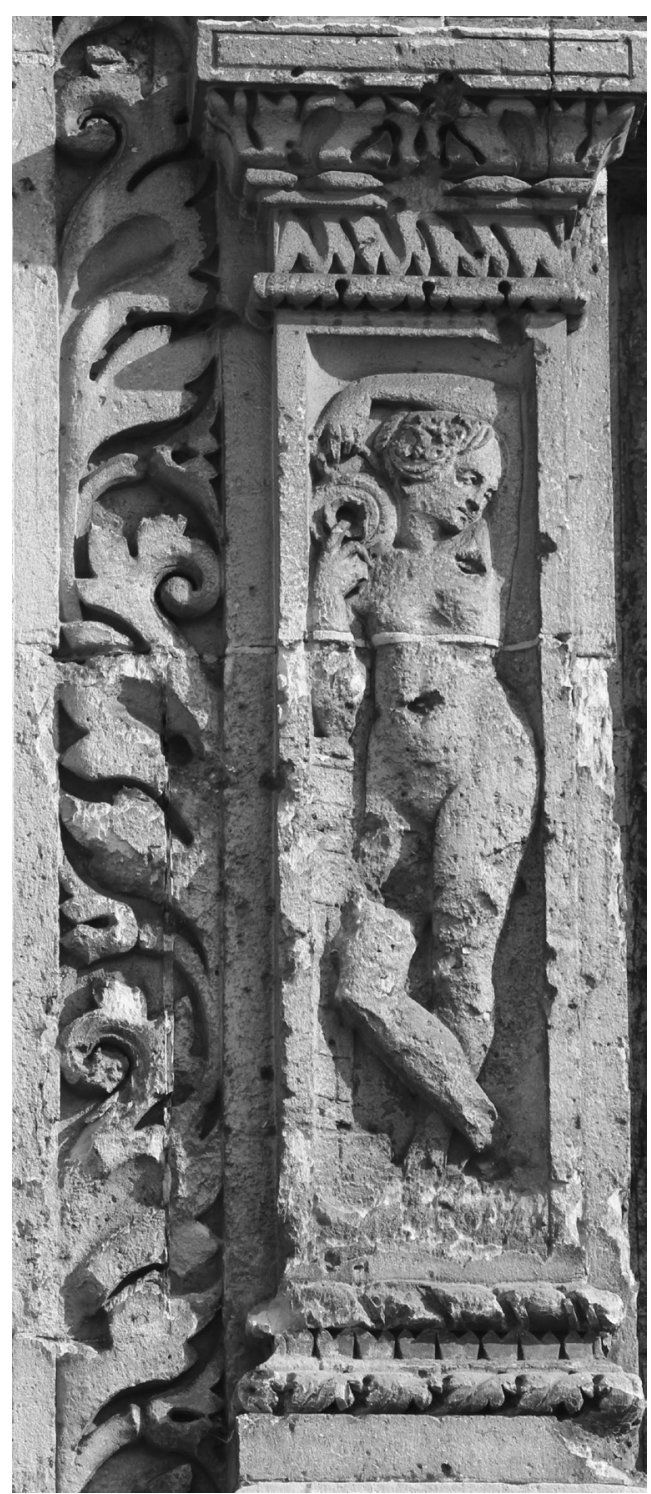

Figure 34 : Rinceau vertical jouxtant une niche de la Porte de Mars à Reims. Cliché Yvan Maligorne. Figure 34: Vertical scroll next to a niche of the Porte de Mars in Reims.

rinceaux du monument baïocasse présentent toutefois une particularité notable : deux tiges secondaires s'échappent très souvent l'une en face de l'autre de la tige principale, dessinant avec elle un motif en forme de trident. On rencontre ce type de motif sur d'assez nombreux pampres de vigne, mais il ne constitue généralement pas un caractère structurant de la composition : ainsi sur un autel de marbre découvert à Langres, on relève de façon très épisodique cette association de trois tiges (Espérandieu IV, 3341). Ce qui frappe à Bayeux, c'est au contraire le caractère presque systématique du procédé. Celui-ci apparaît sur des blocs d'Amiens 
(Walter, 1975, pl. XII) et de Genainville (Mitard, 1993, p. $\left.160, \mathrm{n}^{\text {os }} 103,104,106,107\right)$, mais nous avons surtout observé ce trait récurrent sur les colonnes du péristyle d'une domus de Vieux, chef-lieu de la civitas voisine des Viducasses. Comme à Bayeux, il s'agit ici d'une véritable "signature " (fig. 35). Le constat invitait naturellement à prolonger la comparaison, qui s'est révélée fructueuse : l'anthémion qui orne l'échine des chapiteaux toscans de ces mêmes colonnes présente des parentés évidentes avec celui qui couronne cer- taines archivoltes de l'arc de Bayeux (fig. 36). Or - et c'est cela qui nous intéresse au premier chef -, la fouille stratigraphique a permis à Pascal Vipard de dater de la période sévérienne la réfection du péristyle et la mise en place de ces colonnes (Vipard 1998, p. 29); cette datation doit de toute évidence être étendue au monument de Bayeux, dont le décor - c'est la seconde conclusion que nous croyons devoir déduire du rapprochement - a été confié à des artisans qui ont aussi travaillé à Vieux. a
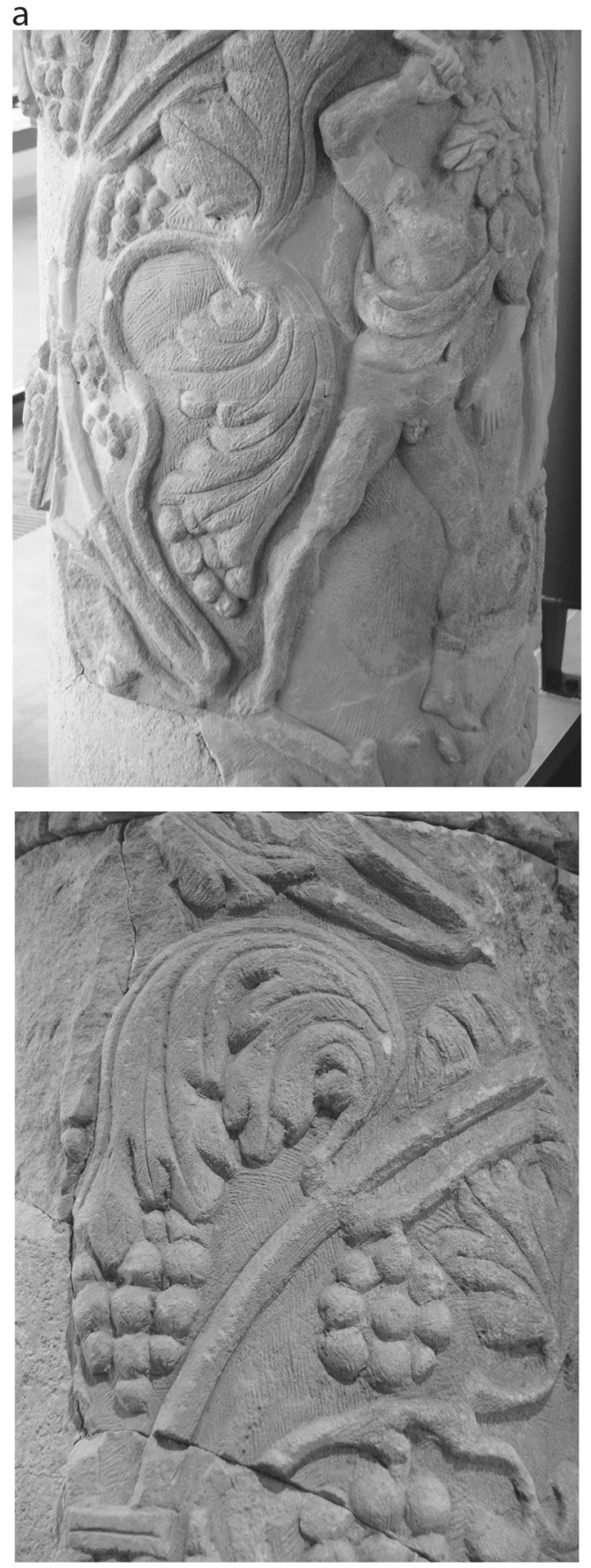

C b
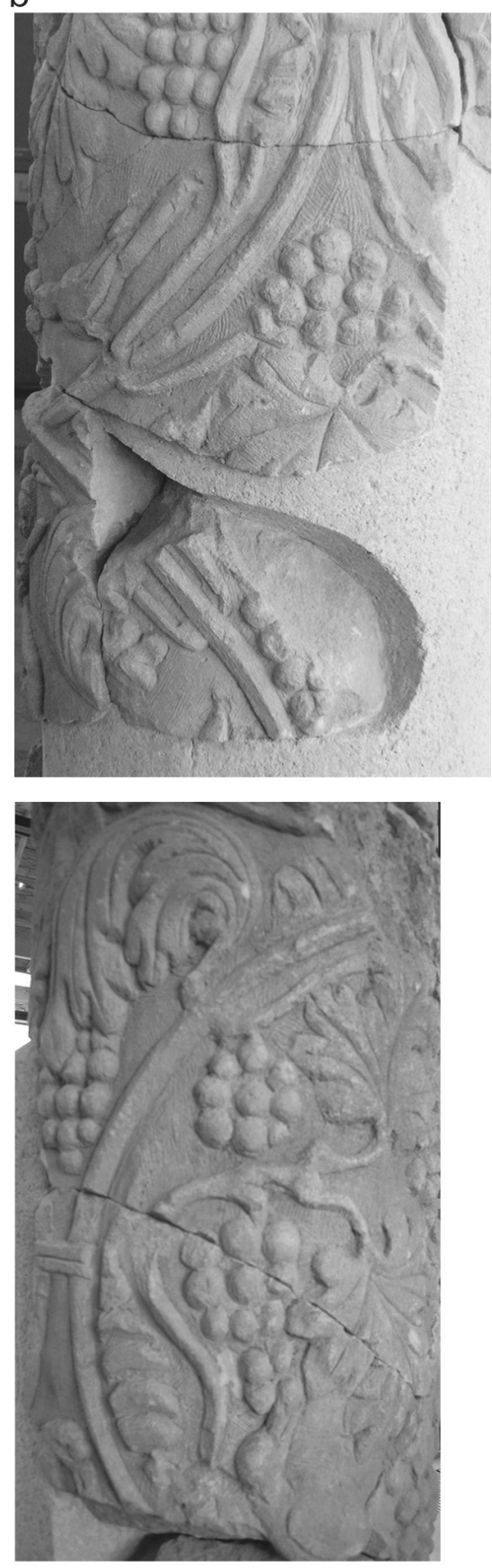

d
Figure 35 : Rinceaux de vigne ornant les colonnes du péristyle de la domus dite du Bas-de-Vieux, à Vieux. Cliché Yvan Maligorne.

Figure 35 : Vine scrolls decorating the peristyle columns of the domus of Bas-deVieux. 


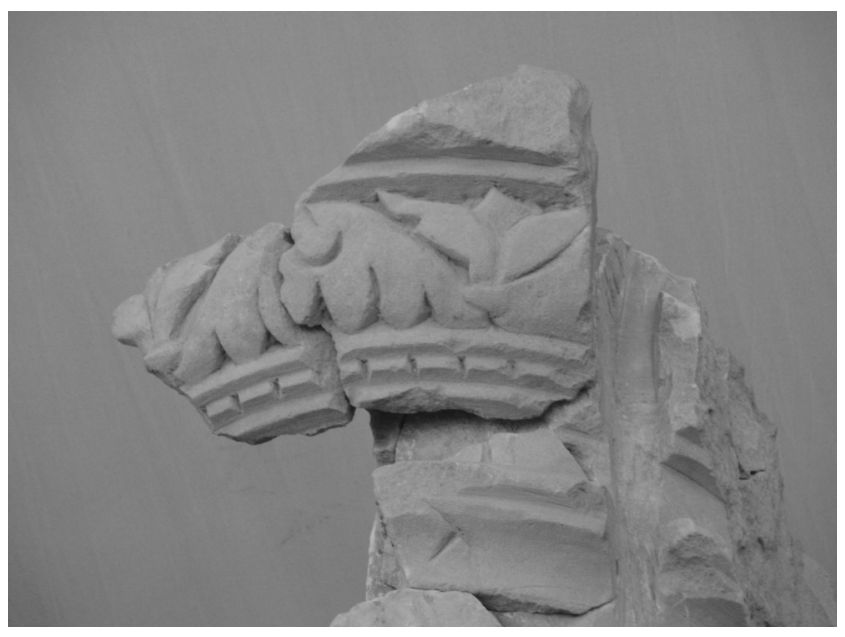

Figure 36 : Chapiteau toscan d'une colonne du péristyle de la domus dite du Bas-de-Vieux. Cliché Yvan Maligorne.

Figure 36: Tuscan capital of one of the peristyle columns of the domus of Bas-de-Vieux.

\section{UN ARC MONUMENTAL À TROIS BAIES}

Ces premières conclusions facilitent l'étude architecturale en orientant la constitution du dossier comparatif. Certes, les blocs conservés sont trop peu nombreux pour que nous proposions une restitution très précise du monument, mais ils nous fournissent un certain nombre d'informations importantes qui peuvent être partiellement complétées grâce à une mise en série, laquelle est rendue plus aisée par l'abondance des occurrences monumentales conservées et publiées.

Les composantes les plus importantes du monument de Bayeux sont des baies couvertes par une voûte (blocs 7-19) reposant sur des pilastres (blocs 1-4); ces baies étaient encadrées par des piles elles-mêmes animées par des colonnes engagées (blocs 5-6). Les claveaux, qui présentent une hauteur et une portée importantes, assurent que les baies constituent les éléments essentiels de l'édifice, en quelque sorte sa raison d'être, et non pas seulement des structures accessoires. Le monument auquel nous avons affaire doit donc bien être rangé dans la catégorie des portes ou arcs monumentaux - nous reviendrons sur ces questions de terminologie quand nous aurons progressé dans l'évocation de la morphologie du monument.

Les éléments essentiels sont donc les claveaux, qui fournissent des informations sur le nombre et les dimensions des baies. Nous avons synthétisé les informations qui les concernent dans un tableau, permettant de les comparer et de les répartir en plusieurs types, chacun correspondant à une baie différente (tableau 1). Nous avons tenu compte du décor des deux archivoltes, de leur couronnement respec- tif et des intrados. Le principe qui a guidé la démarche est que chaque baie doit être soumise à un système ornemental cohérent, et que chaque système atteste l'existence d'une baie distincte. Il faut naturellement prêter attention au fait qu'un intrados ou un bandeau d'archivolte peut présenter un décor compartimenté, faisant par exemple alterner décor végétal et décor figuré - ce type de schéma est en tout cas illustré par des frises d'entablement (Tardy, 2005, fig. 68, $69,72,73)$. Ce qui est certain en revanche - et c'est un critère décisif pour la classification et la répartition des blocs -, c'est que les moulures de couronnement, elles, ne peuvent être que continues, présentant le même profil et un décor au moins voisin sur tout leur parcours. Tenant compte de ces critères, ce sont bien trois baies que documentent les blocs conservés.

La première (A) est définie par des claveaux portant sur une face un rinceau de vigne couronné par un anthémion, sur la face opposée, un ample anthémion, la moulure de couronnement étant inconnue; l'intrados est décoré d'un rang de caissons carrés frappés de fleurons, caissons encadrés par deux tresses.

La deuxième baie (B) est attestée par des claveaux portant sur une face un décor figuré couronné par une tresse sur bandeau, sur l'autre face un rinceau couronné par des feuilles inclinées sur bandeau; l'intrados est agrémenté d'un décor végétal, peut-être un candélabre.

La troisième baie (C) n'est représentée avec certitude que par un bloc ( $\left.\mathrm{n}^{\circ} 17\right)$, mais son décor, ou plutôt les associations qu'il met en œuvre, sont incompatibles avec les deux autres systèmes, dont l'existence est fermement établie. Ce bloc porte sur une face une composition végétale (deux rinceaux ou plus probablement une succession de rinceaux courts) couronnée par une file de feuilles et de joncs, et sur l'autre face un anthémion couronné par un autre anthémion; l'intrados est revêtu d'un décor au moins partiellement figuratif.

Deux blocs, trop fragmentaires ( $\mathrm{n}^{\text {os }} 18$ et 19$)$, ne peuvent être attribués avec certitude à un type et sont théoriquement compatibles avec deux systèmes (A et C).

Ces baies se distinguent aussi par leurs dimensions. Avant de livrer les résultats auxquels nous pensons être parvenus, il faut dire un mot des difficultés de calcul. Les blocs ne sont jamais très longs et ne conservent donc pas une grande portion de courbure; il nous est très vite apparu que leurs faces latérales ne sont pas disposées radialement par rapport au centre des arcs, comme cela devrait théoriquement être le cas. Nous avons dû nous en remettre à des méthodes empiriques, en essayant de les croiser pour contrôler les résultats, qui ne sauraient prétendre à une exactitude parfaite.

Le constat le plus important porte sur l'existence de deux portées différentes. Deux de ces trois baies (B et $\mathrm{C}$ ) présentaient des dimensions identiques et plutôt modestes, 


\begin{tabular}{|c|c|c|c|c|c|c|c|}
\hline & $\begin{array}{c}\mathrm{N}^{\circ} \mathrm{du} \\
\text { bloc }\end{array}$ & Archivolte 1 & $\begin{array}{l}\text { Couronnement de } \\
\text { l'archivolte } 1\end{array}$ & Archivolte 2 & $\begin{array}{l}\text { Couronnement de } \\
\text { l'archivolte } 2\end{array}$ & Intrados & $\begin{array}{c}\text { Prof. } \\
\text { à l'intrados }\end{array}$ \\
\hline Type A & 7 & Vigne & Anthémion sur talon & Anthémion ample & - & Fleurons entre tresses & \\
\hline Type A & 8 & Vigne & Anthémion sur talon & - & - & Fleurons entre tresses & $65,2 \mathrm{~cm}$ cons. \\
\hline Type A & 9 & Vigne & Anthémion sur talon & - & - & - & \\
\hline Type A & 10 & - & - & Anthémion ample & - & - & \\
\hline Type A & 11 & Vigne & - & - & - & Fleuron entre tresses & $68 \mathrm{~cm}$ \\
\hline Type A & 12 & - & - & Anthémion ample & - & Fleurons entre tresses & \\
\hline Type A & 13 & - & - & - & - & Fleurons entre tresses & \\
\hline \multirow{2}{*}{$\begin{array}{l}\text { Type B } \\
\text { Incompatible } \\
\text { avec A }\end{array}$} & 14 & Griffon & Tresse sur bandeau & Rinceau & $\begin{array}{c}\text { Feuilles inclinées sur } \\
\text { bandeau }\end{array}$ & $\begin{array}{l}\text { Décor végétal avec } \\
\text { masques suspendus }\end{array}$ & $69,5 \mathrm{~cm}$ \\
\hline & 15 & Griffon & Tresse sur bandeau & Rinceau & $\begin{array}{c}\text { Feuilles inclinées sur } \\
\text { bandeau }\end{array}$ & Candélabre végétal & \\
\hline Type B & 16 & $\begin{array}{c}\text { Décor figuré : } \\
\text { pedum et anneau }\end{array}$ & Tresse sur bandeau & Rinceau & - & $\begin{array}{c}\text { Décor végétal (can- } \\
\text { délabre?) }\end{array}$ & $68 \mathrm{~cm}$ \\
\hline $\begin{array}{l}\text { Type C } \\
\text { Incompatible } \\
\text { avec A et B }\end{array}$ & 17 & Rinceau & $\begin{array}{l}\text { Feuilles d'acanthe et } \\
\text { joncs sur doucine }\end{array}$ & Anthémion ample & Anthémion sur talon & Amour nu & \\
\hline $\begin{array}{l}\text { Type A ou C } \\
\text { Incompatible } \\
\text { avec B }\end{array}$ & 18 & - & - & Anthémion ample & Anthémion sur talon & & \\
\hline $\begin{array}{l}\text { Type A ou C } \\
\text { Incompatible } \\
\text { avec B }\end{array}$ & 19 & & & & Anthémion sur talon & & \\
\hline
\end{tabular}

Tableau 1 : Classement des blocs de claveau en fonction du décor de leurs archivoltes et de leur intrados.

Table 1: Inventory of voussoirs in relation to the decor of their archivolts and their lower surface.

leur portée s'élevant à environ trois mètres - une portée de 10 pieds semble ici très probable. La troisième baie $(\mathrm{A})$, plus importante, avait une portée d'environ cinq mètres.

Le bloc 20 pose des problèmes difficiles, qui doivent faire l'objet de développements spécifiques : le bandeau qui sépare dans le sens vertical les deux panneaux figurés est courbe et s'infléchit vers la gauche. Nous en avons d'abord déduit que le bloc intégrait une partie d'une archivolte, ornée d'un triton, et la partie inférieure de l'écoinçon droit; la disposition relative des lits de pose et d'attente semblait conforter cette hypothèse, puisque, si le premier est horizontal, le second est déversé (fig. 37). Une difficulté subsiste, en l'espèce la forme du couronnement : ce bandeau lisse serait compatible avec les couronnements des deux archivoltes de la baie B (ornés l'un d'une tresse sur bandeau, l'autre de feuilles inclinées sur bandeau), mais l'amplitude de la courbure ne semble guère compatible qu'avec la baie $\mathrm{A}$; l'une de ses archivoltes est couronnée par un talon; le couronnement de l'autre est inconnu, mais le bandeau de l'archivolte est décoré d'un anthémion. On le voit, aucune solution ne s'impose d'emblée, et nous avons préféré ne pas intégrer ce bloc à la restitution graphique.
C'est donc un monument à trois baies qui doit être restitué, la baie centrale possédant un plus grand développement que les deux baies latérales. Les archivoltes de ces baies reposaient sur les pilastres ornés de rinceaux de vigne, dont les blocs conservés nous apprennent qu'ils flanquaient les ouvertures. Un des pilastres (blocs 1 et 2) développe un système ornemental plus complexe et plus soigné que l'autre, et nous l'avons attribué à l'encadrement de la baie centrale. Les deux tambours qui nous sont parvenus (blocs 5 et 6 ) suffisent à établir que le monument était aussi structuré par des colonnes engagées : accolées aux piles, elles s'élevaient au moins jusqu'au sommet des voûtes pour supporter un entablement horizontal, lequel devait être surmonté d'un attique.

Nous avons longuement hésité avant de donner une traduction graphique à nos observations et hypothèses, avant de nous y résoudre finalement pour que le discours soit plus facile à suivre et pour donner une idée plus précise des résultats auxquels nous pensons être parvenus (fig. 38. Les blocs utilisés pour cette restitution sont rassemblés sur la fig. 39). On peut donc tenir pour assurée l'existence des trois baies, des pilastres, des colonnes, mais nous ne savons rien de la largeur des piles, fixée ici au minimum admissible; la position exacte de presque tous les blocs est hypothétique, étant 


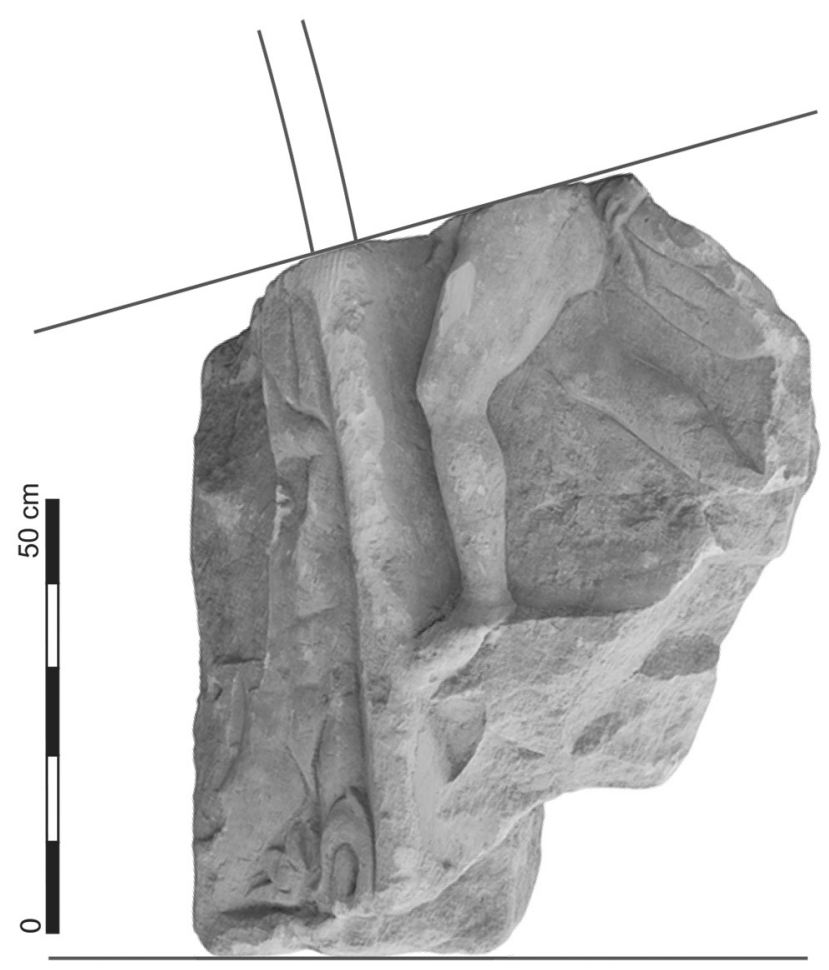

Figure 37 : Le bloc 20, avec mise en valeur des lits de pose et d'attente et de la moulure courbe. Cliché et DAO Annabelle Cocollos, CG 14.

Figure 37: Block 20: the beds and the curvilinear moulding are highlighted.

entendu que la rareté des éléments jointifs interdit d'établir des séquences; nous ne savons rien des parties hautes du monument : si l'existence d'un entablement complet peut être tenue pour certaine, celle d'un attique est simplement probable; on remarque enfin que le problème des angles n'a pas été affronté, tant les solutions possibles sont nombreuses. Nous ne sommes absolument pas assurés que le monument était indépendant sur le plan structurel, qu'il n'était pas intégré à un ensemble plus vaste.

Dans le détail, nous ignorons tout de certaines zones clefs. Le bloc 1 nous apprend qu'au moins l'une des baies était encadrée par un dispositif complexe : les pilastres de façade, ornés de rinceaux de vigne, étaient flanqués à l'ouverture de la baie de pilastres ornés de rinceaux acanthisés. Un tel schéma implique l'existence, au sommet des supports, de blocs complexes assurant la transition entre pilastres et archivolte; ces blocs associaient probablement deux chapiteaux différents. Nous ne savons rien non plus des parties basses du monument, et si nous avons retenu la solution architectonique la mieux attestée, avec des colonnes reposant sur un socle tandis que les pilastres s'élèvent pratiquement depuis le sol, elle n'est pas la seule envisageable. Considérant l'absence de colonnes libres
- elles seraient totalement incompatibles avec les colonnes engagées, et quand des arcs présentent des colonnes libres, ce sont des pilastres qui leur font écho sur la paroi - nous avons préféré restituer un entablement et un attique entièrement rectilignes, dépourvus donc de ces ressauts qui animent de nombreux monuments.

Cette animation plastique très sommaire des façades a pour corollaire un phénomène qu'élude la vue unique que nous proposons, à savoir la très faible profondeur $\mathrm{du}$ monument, qui ne dépasse pas $75 \mathrm{~cm}$; cette caractéristique, encore accentuée par son étirement, l'assimile à une simple façade monumentale plutôt qu'à un volume autonome. Il n'est d'ailleurs pas impossible qu'une des faces ait été privilégiée : si les claveaux sont tous ornés des deux côtés, plusieurs blocs ne présentent de décor que sur l'un des deux longs côtés, l'autre semblant avoir toujours été lisse; c'est le cas des blocs 3 et 20 , qui ne portent pas la moindre trace de sculpture au revers. L'état de conservation de ces faces interdit toute conclusion définitive, mais il est très vraisemblable que l'ensemble du monument n'ait pas été orné, ce qui, de fait, conduit à définir une face principale et une face secondaire.

Faible profondeur et mise en valeur d'une des faces : ces deux caractéristiques s'observent très fréquemment sur des arcs qui ne constituent pas des monuments isolés mais sont structurellement intégrés à des ensembles plus vastes auxquels ils donnent accès. C'est ce qu'a démontré Caroline Blonce dans un travail de grande ampleur qui s'attache à recenser tous les arcs des $\mathrm{II}^{\mathrm{e}}$-IV $\mathrm{IV}$ siècles; elle donne à ces structures le titre de "portes triomphales ", tout en soulignant - nous y reviendrons - que les inscriptions les qualifient d'arcus (Blonce, 2008, p. 49-57). Ces monuments diaphragmes sont particulièrement bien représentés dans les provinces africaines, ce qui ne fait que refléter la particulière densité des arcs dans ces régions. Certains sont moins épais encore que celui de Bayeux : l'arc de Vazi Sarra (ibid., $\mathrm{n}^{\circ} 156$ ) est épais de $0,50 \mathrm{~m}$; celui de Thuburbo Maius ( $\mathrm{n}^{\circ} 88$ ), de $0,52 \mathrm{~m}$; les deux arcs de Thimida Bure ( $\mathrm{n}^{\text {os }} 117$ et 124), de 0,57 et $0,61 \mathrm{~m}$.

La réduction de la profondeur sur les monuments des $\mathrm{II}^{\mathrm{e}}$ et $\mathrm{III}^{\mathrm{e}}$ siècles relève d'une tendance lourde, qui se manifeste aussi dans les Gaules : l'arc d'Aix-les-Bains n'est épais que de 0,75 m (Prieur, 1982, p. 461-462; Küpper-Böhm, 1996, p. 129-135), sans toutefois posséder l'ampleur de celui que nous restituons (fig. 40); les structures massives de l'arc de Besançon - excluant donc les colonnes libres qui se projettent en avant de l'édifice - sont profondes de 1,05 $\mathrm{m}$, la profondeur totale du monument ne dépassant pas 2,11 mètres (Walter, Bruchet 1986, vol. II, fig. 5); l'arc de Dativius Victor, à Mayence, n'est profond que de 0,70 m et une seule de ses faces est ornée (Frenz 1981, 


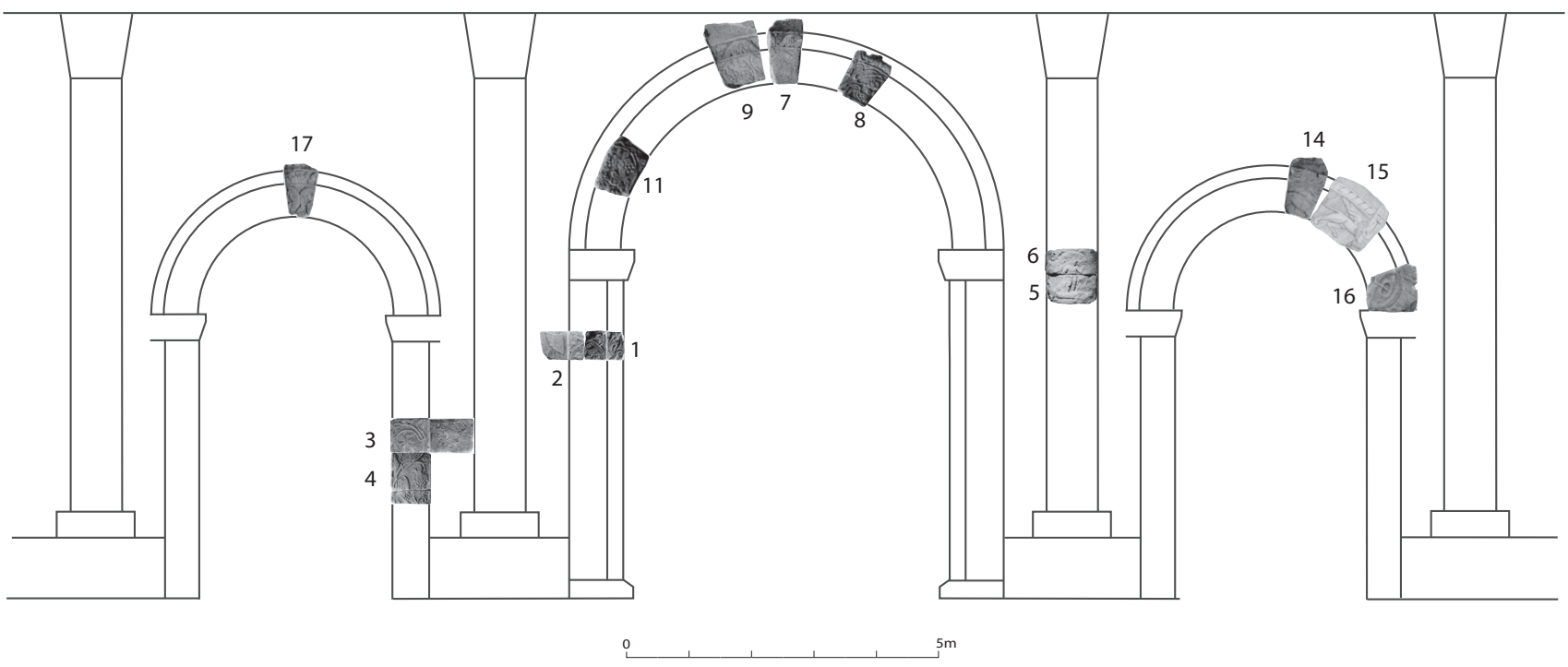

Figure 38 : Évocation de la face principale de l'arc. Dessin Yvan Maligorne, DAO Annabelle Cocollos.

Figure 38: Evocation of the main face of the arch.

p. 227; fig. 41); il s'ouvrait d'ailleurs au sein d'un portique, mentionné par la dédicace (ILS, 7080 : arcum et porticus), ce qui en fait peut-être une de ces structures intégrées qu'énumère $\mathrm{C}$. Blonce. La fouille récente d'un péribole de sanctuaire à Pont-Sainte-Maxence par V. Brunet-Gaston (Inrap) nous offre un élément de comparaison intéressant : l'ample façade du sanctuaire, composée d'arcades encadrées par un entablement droit, longue de 90 mètres et haute de 9,50 mètres, n'était en effet épaisse que d'un mètre, ce qui expliquerait d'ailleurs son basculement peu de temps après sa construction.

L'arc de Bayeux, qu'il ait été ou non indépendant sur le plan structurel, avait très probablement une fonction de limite : il s'agissait de souligner et marquer un passage, d'annoncer un espace ou, au contraire, de lui offrir une toile de fond.

Le choix d'un terme précis pour le désigner est difficile, en ce qu'il implique des hypothèses fonctionnelles ${ }^{22}$. Cependant, le terme d'arcus l'emporte très largement dans la documentation épigraphique : porta est lié aux portes s'ouvrant dans les remparts, et apparaît très souvent conjointement à murus; janus est plus rarement employé, et d'abord

22. Pour la terminologie, voir Gros, 2011, p. 56-57; Blonce, 2008, p. 66-75. dans la documentation du $\mathrm{I}^{\text {er }}$ siècle $^{23}$; quant au terme fornix, il a un sens technique, se rapportant à une baie ou une voûte. Il semble donc légitime d'employer ici le terme d'arc, sans autre qualificatif et en gardant bien à l'esprit qu'il a un caractère plutôt générique. L'ignorance dans laquelle nous sommes des circonstances de sa construction interdit d'aller plus loin : la dédicace est perdue, et son programme iconographique n'est connu que par de maigres lambeaux.

\section{UNE ICONOGRAPHIE DOMINÉE PAR UNE THÉMATIQUE DIONYSIAQUE (FIG. 42)}

Le monument de Bayeux était revêtu d'une riche décoration sculptée, qui, pour autant que nous puissions en juger, n'épargnait aucune composante : non seulement les supports verticaux (colonnes et pilastres), les claveaux et l'intrados des voûtes étaient couverts de reliefs, mais les piles elles-mêmes étaient ornées, au moins sur l'une des deux faces principales. Cela renvoie semble-t-il à une tendance profonde, puisque les autres arcs monumentaux connus en Gaule et datant de la même période développent des programmes icono-

23. Pour les emplois du mot, voir Blonce, 2008, p. 69-71 : il est complètement absent des inscriptions des $\mathrm{II}^{\mathrm{e}}-\mathrm{IV}^{\mathrm{e}}$ siècles. 

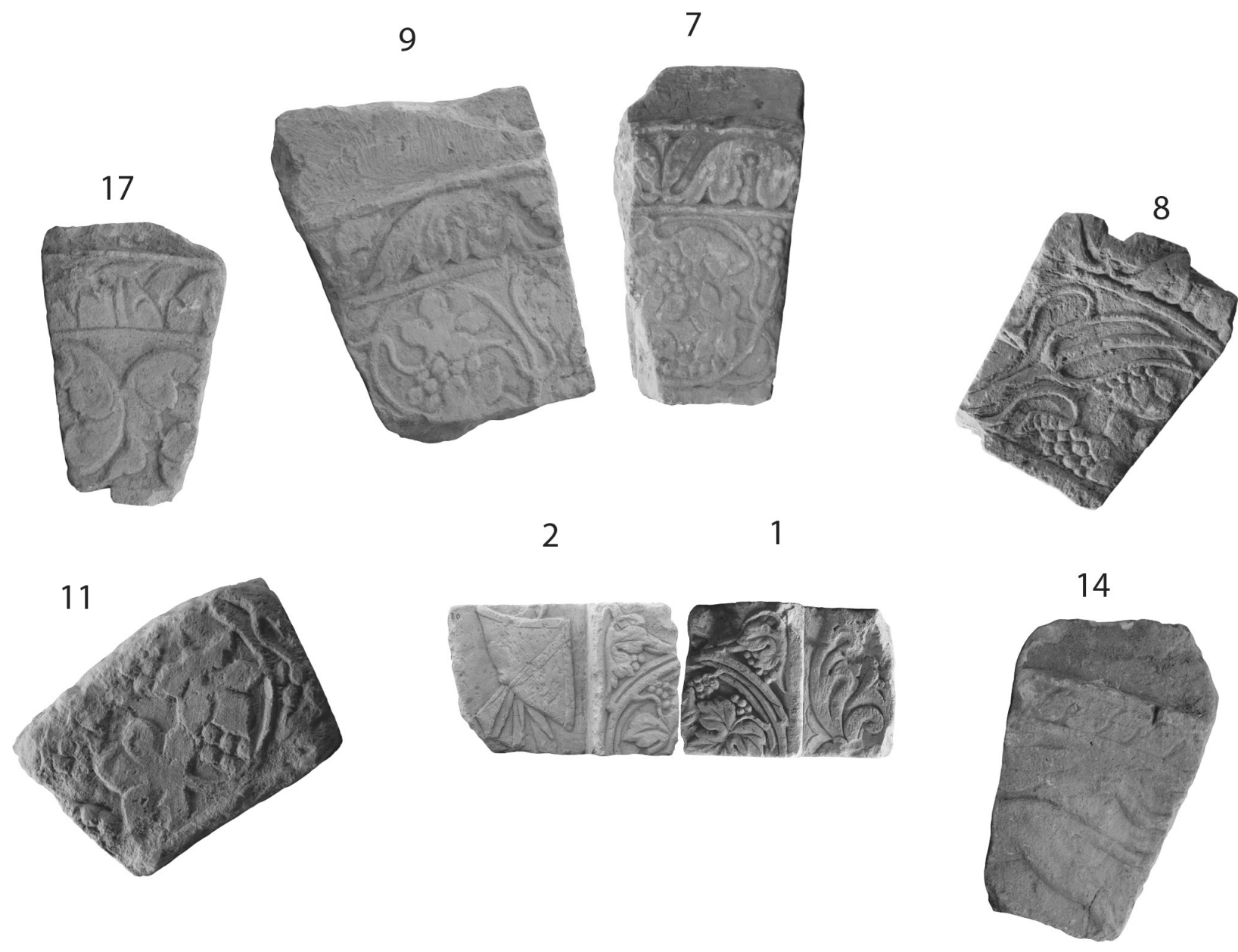

3
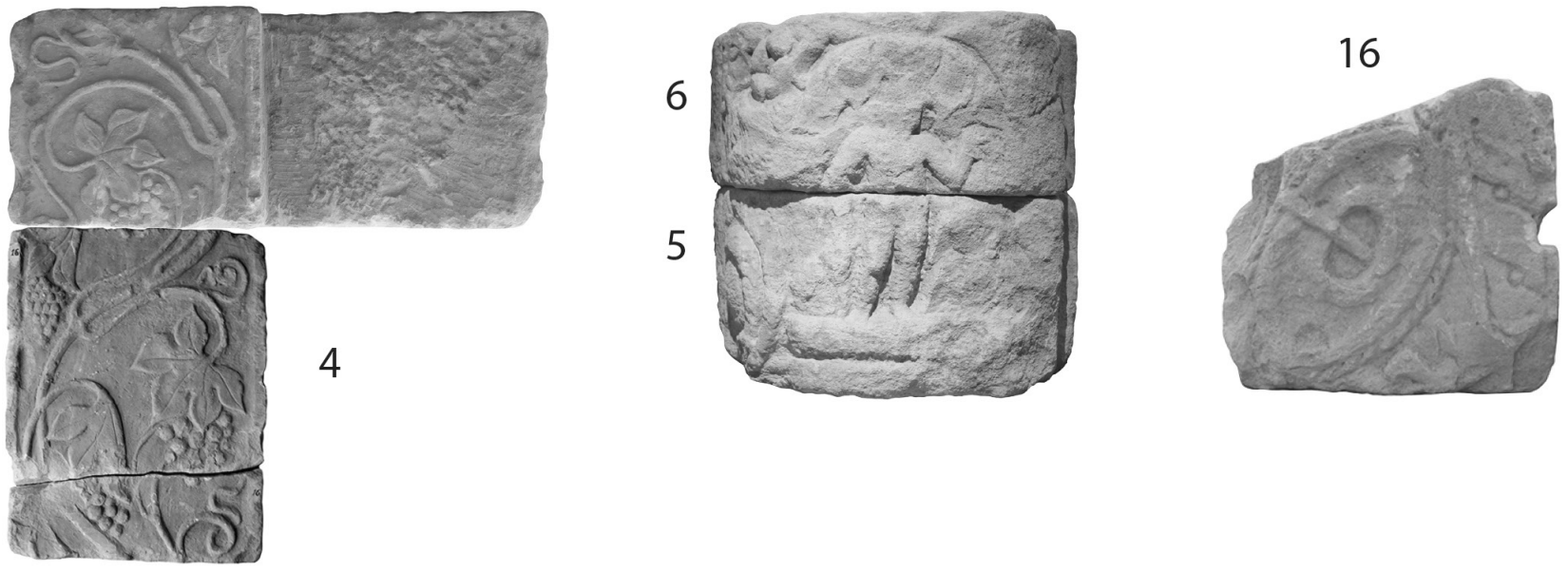

$1 \mathrm{~m}$

Figure 39 : Les blocs mis à contribution dans la reconstitution. Clichés Annabelle Cocollos, CG 14. Figure 39: The blocks contributing to the restoration. 


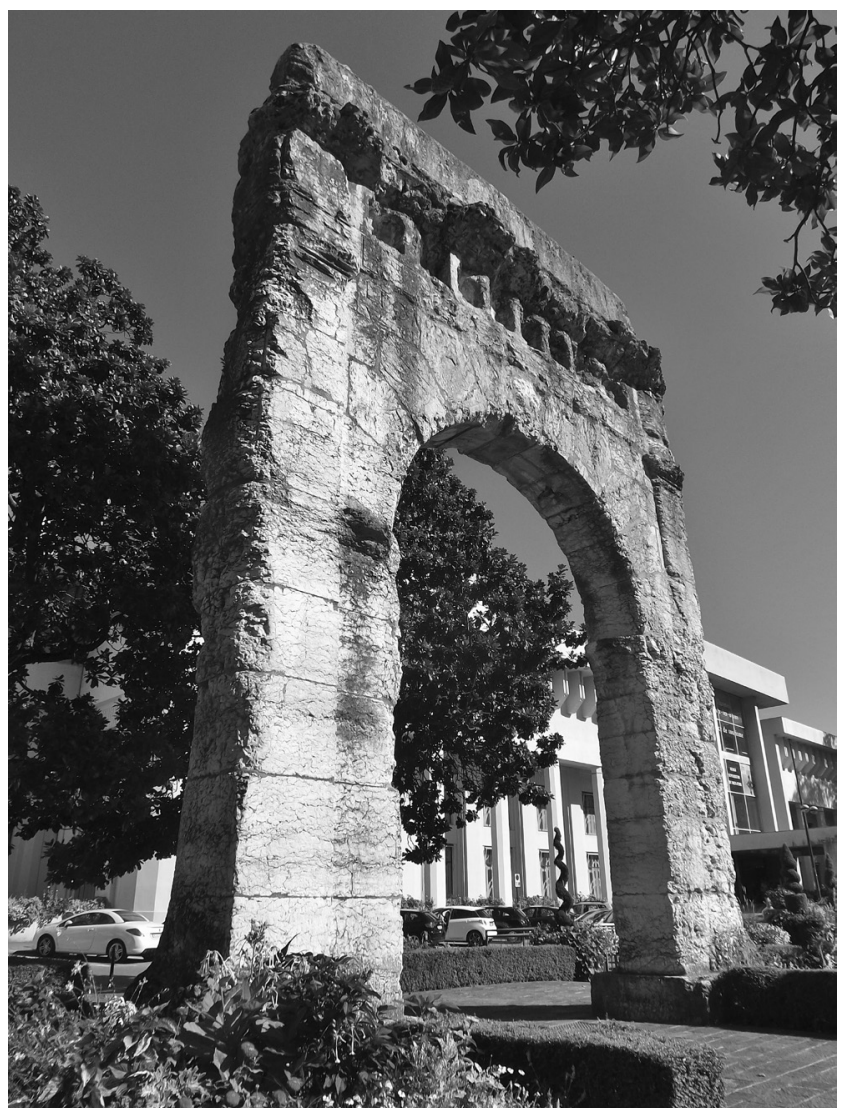

Figure 40 : L'arc d'Aix-les-Bains. Cliché Internet : [http://commons.wikimedia.org/wiki/File:Arc_de_Campanus_et_thermes_nationaux. JPG? uselang $=f r]$.

Figure 40: The arch of Aix-les-Bains.

graphiques denses : la Porte Noire de Besançon, bien sûr (Walter, Bruchet, 1986, complété par Walter, 2006; fig. 43), mais encore la Porte de Mars à Reims (Lefèvre, 1985; fig. 44) et l'arc de Dativius Victor à Mayence (Frenz, 1981; fig. 41). On rappelle fréquemment qu'un décor abondant constitue l'une des caractéristiques saillantes des arcs gaulois et ce, dès la période augusto-tibérienne (Walter, Bruchet, 1986, p. 263-264); mais même le décor de l'arc d'Orange, pourtant exceptionnellement riche, est encore structuré en grands panneaux très lisibles, épargne les colonnes et laisse subsister de grandes plages lisses. Les arcs de la deuxième moitié du $\mathrm{II}^{\mathrm{e}}$ siècle et de l'époque sévérienne relèvent de tendances différentes, qui se manifestent sur d'autres types monumentaux ${ }^{24}$.

Nous ne connaissons de cette décoration que des bribes. La restitution graphique rend sensible - si elle a un mérite, c'est celui-là - le caractère très lacunaire de notre documentation, qui ne représente qu'une infime partie de l'édifice. Si frus-

24. La "façade des thermes de Sens » en constitue la meilleure illustration (Adam et al., 1987), mais le décor des tombeaux et des maisons porte la marque de ces tendances.

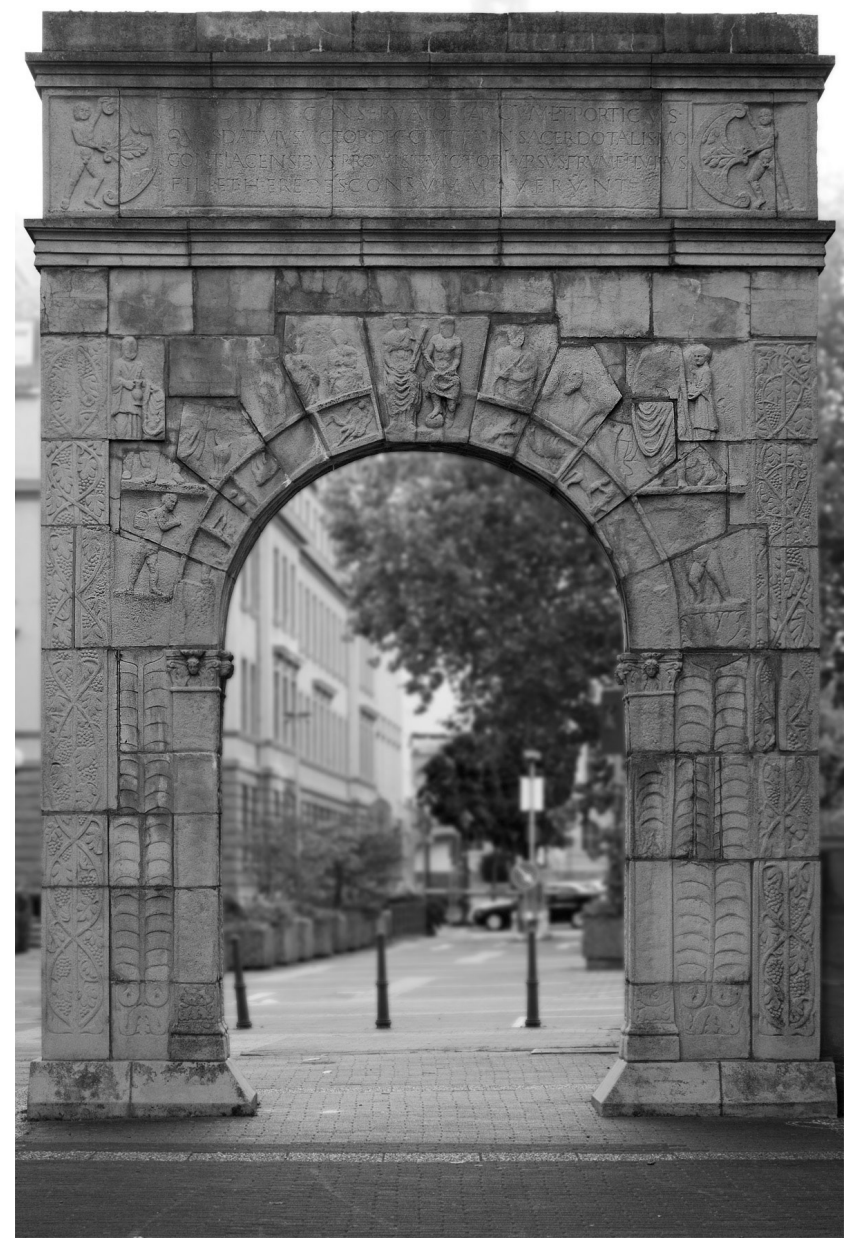

Figure 41 : Restitution de l'arc de Dativius Victor, à Mayence, avec moulage des blocs originaux. Cliché Internet : [http://commons. wikimedia.org/wiki/File:Dativius_Victor_Bogen_1.jpg? uselang=fr]. Figure 41: Restitution of the Dativius Victor arch, in Mainz, with cast of the original blocks.

trants qu'ils soient, ces lambeaux d'iconographie constituent avec l'architecture elle-même notre seul angle d'approche sur la signification du monument, sur le message qu'il entendait délivrer. On n'oubliera pas, cependant, que les éléments les plus importants de cette iconographie étaient les statues qu'accueillait l'attique et qui constituaient - sources littéraires et épigraphiques sont claires à ce propos - l'une des raisons d'être du monument (Blonce, 2008, p. 63-64 et 283-287).

L'échantillon n'est pas nécessairement représentatif et la prudence doit prévaloir au moment de l'interprétation. On ne peut cependant manquer de relever qu'une thématique dominante se dégage de la plupart des reliefs. 
a

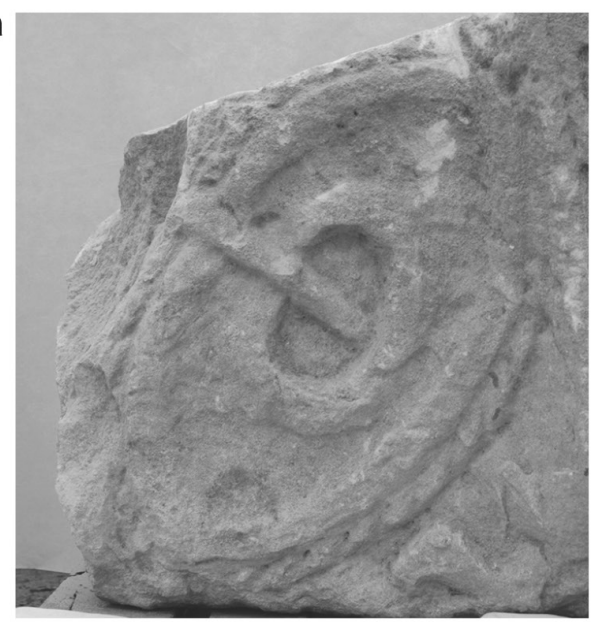

C

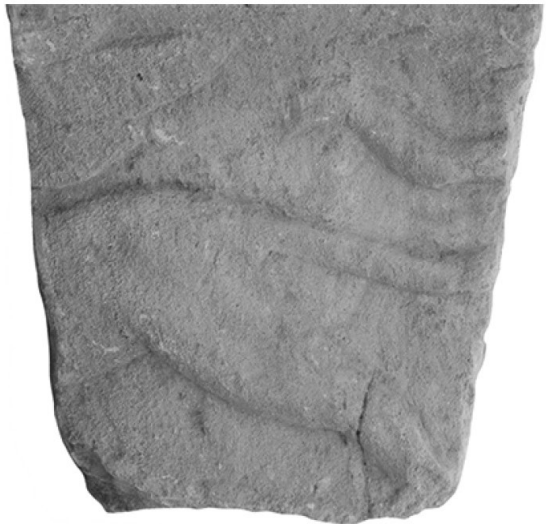

b

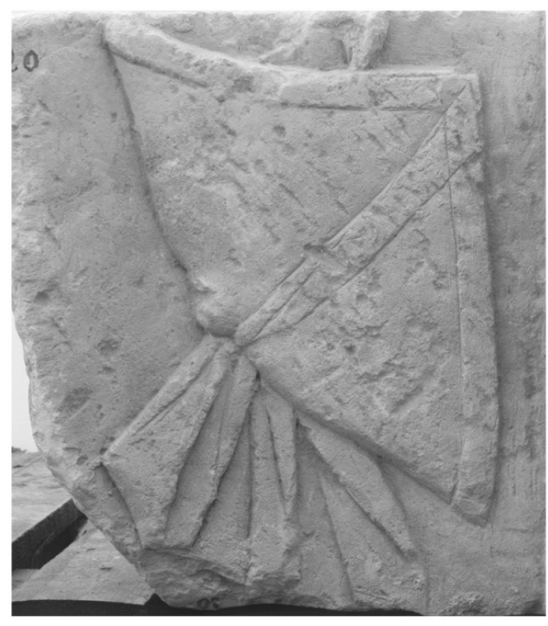

d

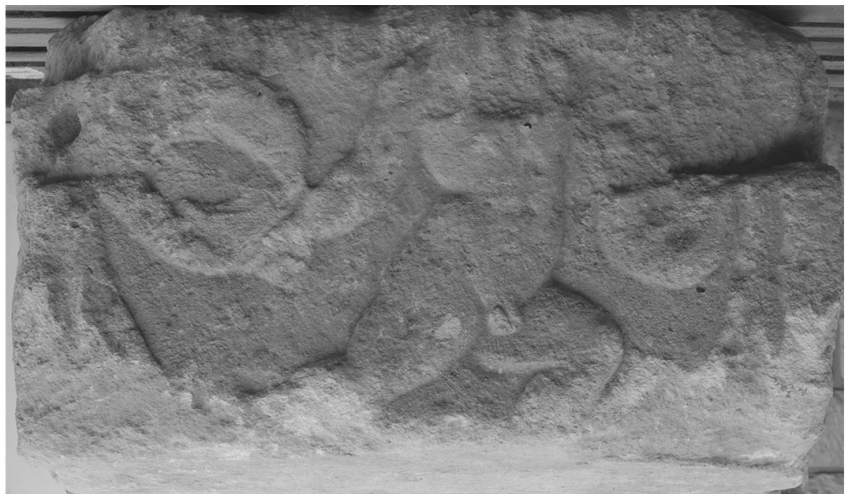

e

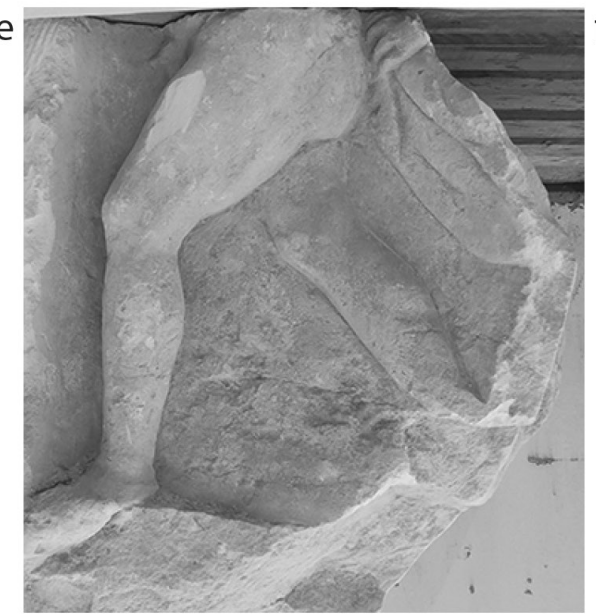

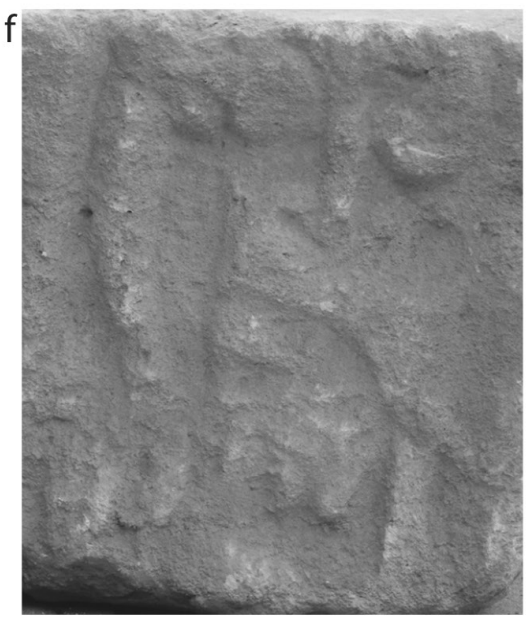

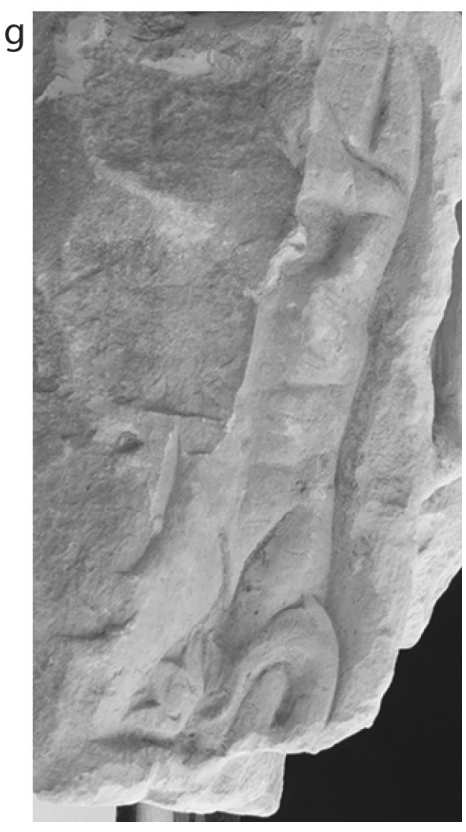

Figure 42 : Le décor figuré de l'arc de Bayeux. (a) Pedum passé dans un anneau, bloc 16. (b) Bouclier suspendu par des rubans, bloc 2. (c) Griffon, bloc 14. (d) Amour, bloc 17. (e) Personnage nu du bloc 20. (f) Têtes suspendues aux tiges d'un candélabre végétal, bloc 14. (g) Triton, bloc 20 (clichés Annabelle Cocollos, CG 14).

Figure 42: The figured decor of the Bayeux arch. (a) Pedum in a ring, block 16. (b) Shield suspended by ribbons, block 2. (c) Griffin, block 14. (d) Amour, block 17. (e) Nude figure block 20. (f) Suspended heads on a vegetal candelabrum, block 14. (g) Triton, block 20. 


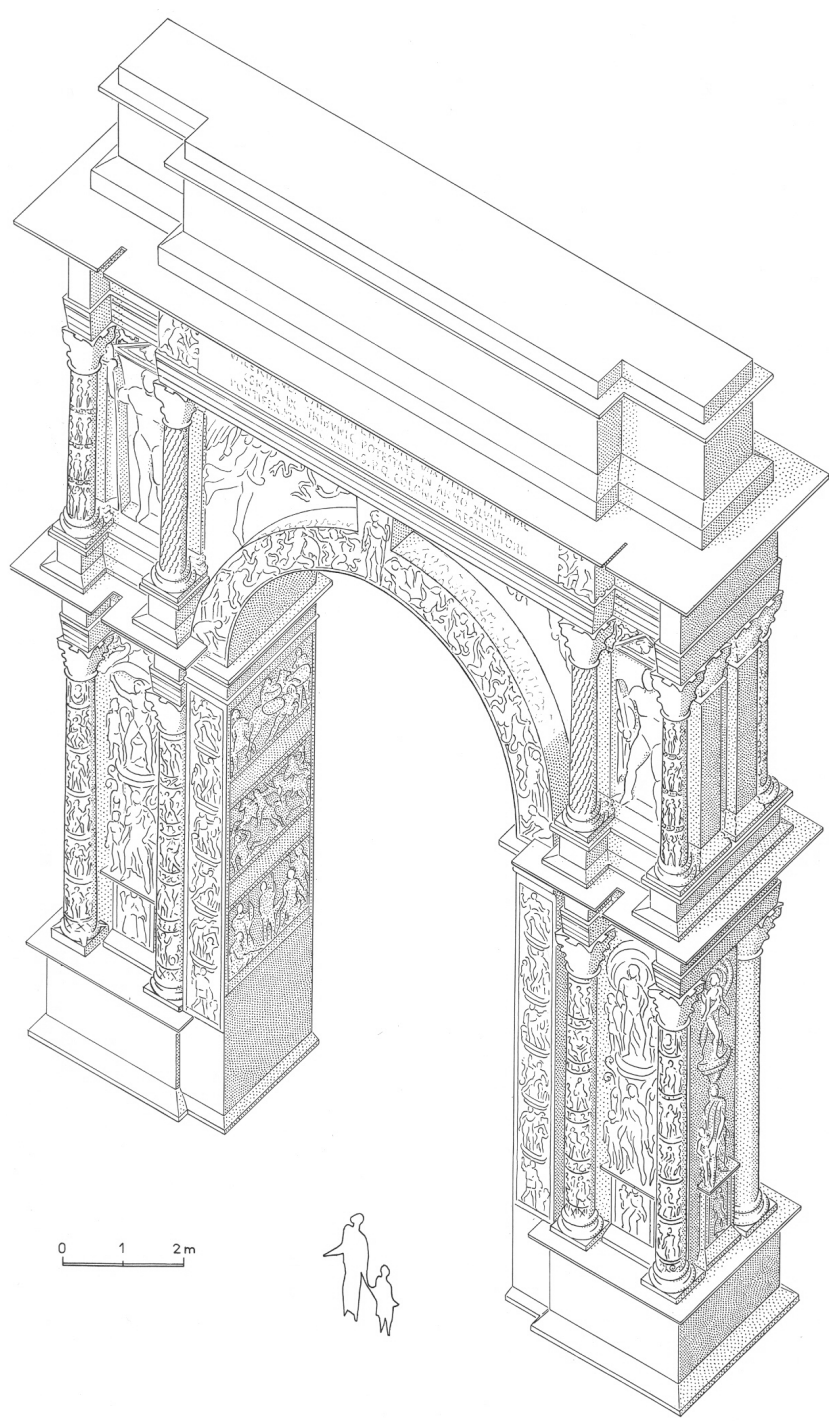

Figure 43 : Vue axonométrique de la Porte Noire de Besançon (d'après Walter et Bruchet 1986). Le dessin donne une idée précise de la profusion ornementale du monument.

Figure 43: Axonometric view of the Porte Noire at Besançon (after Walter and Bruchet 1986). The drawing gives an idea of just how ornate the monument was.

Beaucoup entretiennent en effet un rapport avec l'univers dionysiaque ${ }^{25}$. C'est le cas d'abord des rinceaux de vigne; s'ils n'ont en certains contextes qu'une valeur générique ${ }^{26}$,

25. Pour l'identification et l'interprétation de ces symboles, voir les remarques méthodologiques de Wyler 2004, p. 933-937.

26. Voir Tassignon 1996, p. 27 : « [...] rinceaux, pampres, feuilles de vigne et de lierre, grappes de raisins, ont constitué [...] pour les artistes un vocabulaire ornemental qui se répandra sur des monuments aussi divers que nombreux, mais qui n’aura plus, avec le dionysisme, qu'un lien ténu. » Voir encore Küpper-Böhm 1996, p. 147 : « Ein konkreter Verweis auf die Sphäre des Dionysos wurde mit diesem Motiv nicht angestrebt, denn solche Saülen kommen im 2. Jh. an allen nur denkbaren Gebaüdetypen leur sens est ici précisé par plusieurs images. Le pedum est un instrument des bergers, que l'on voit très souvent dans la main des satyres, et c'est l'un des attributs dionysiaques les plus courants (Tassignon, 1996, p. 94); il est très souvent, comme ici, passé dans un anneau (fig. 42a) ${ }^{27}$. Le bouclier suspendu par des rubans (fig. 42b) se rattache au même registre sémantique : transcrit ici sur une paroi, il apparaît plus fréquemment sur des fûts de colonnes parcourus par un réseau de rubans auxquels sont accrochés des instruments dionysiaques explicites, mais aussi des boucliers ${ }^{28}$. C'est encore au monde dionysiaque qu'il faut rattacher les deux griffons ornant l'archivolte de la baie B (fig. 42c) : à l'origine compagnon d'Apollon, le griffon est associé dès le $\mathrm{IV}^{\mathrm{e}}$ siècle avant notre ère au monde de Dionysos (Delplace, 1980, p. 372-376; Gaggadis-Robin, 2005, p. 201). À l'époque impériale, on le retrouve indifféremment associé à l'un et l'autre dieu, comme le montrent par exemple les urnes funéraires, sur lesquelles on voit des griffons accostés tantôt à ces symboles apolliniens que sont le trépied et la lyre (Sinn, $1987, n^{\text {os }} 412$ et 413), tantôt à des rinceaux de vigne ou de lierre, végétaux de Bacchus (ibid., $\mathrm{n}^{\text {os }} 298$, 404). Les deux griffons étaient liés par la queue, selon un schéma bien documenté pour de nombreux monstres; les créatures accolées - griffons, mais aussi dauphins, monstres marins - sont fréquemment séparées par des objets : conques, candélabres, canthares $^{29}$. Le bloc 16, orné du pedum, montre que l'ensemble de l'archivolte n'était pas orné d'une composition de griffons adossés, et les deux monstres documentés ne sont peut-être que la citation ponctuelle d'un carton très répandu, ici insérée dans une composition plus variée, juxtaposant différents symboles dionysiaques.

Le petit Amour (fig. 42d) est plus ambigu : ce petit personnage trouve sa place dans des contextes extrêmement variés, parfois dionysiaques, certes, mais sans que ce soit systématique (Blanc, Gury 1986, en part. p. 1045 et 1047 1048; Gury, 2004). En l'espèce, nous ne disposons que de ce seul bloc pour toute la baie dont provient l'Amour,

vor. Sie entsprechen somit nur im allgemeinsten Sinne der gleichzeitligen Mode dionisischer Themen. "

27. Espérandieu IV, 3233 (Langres; sur le panneau voisin se trouve un masque), 3531 (Dijon); V, 4375 (Lunette d'Arçon). Le motif est parfois utilisé isolément, entre les modillons des corniches : Numrich 1997, pl. 20.1 (pilier d'Iphigénie) et 24.1-2. Les exemples pourraient être multipliés.

28. La liste de ces colonnes est longue. Dans le Nord-Ouest de la Gaule, on citera celles de Rouen (Follain et al., 1996, p. 6-14, avec clichés et dessins) et Lillebonne (Espérandieu, IV, 3014 et 3213); pour un exemple particulièrement riche et varié sur le plan iconographique, voir Terrer $e t a l$., $2003, n^{\circ} 415$. Les systèmes d'attache, avec des rubans épais, sont toujours identiques.

29. Voir par exemple Pensabene 1996, p. 248-251, pour des couples de griffons séparés par des candélabres, sur la frise du temple du divin Antonin, sur le forum Romanum. Pour les Gaules, parmi de très nombreux exemples, voir Mitard, 1993, p. 67; Bossert, 1998, pl. 20-30. 


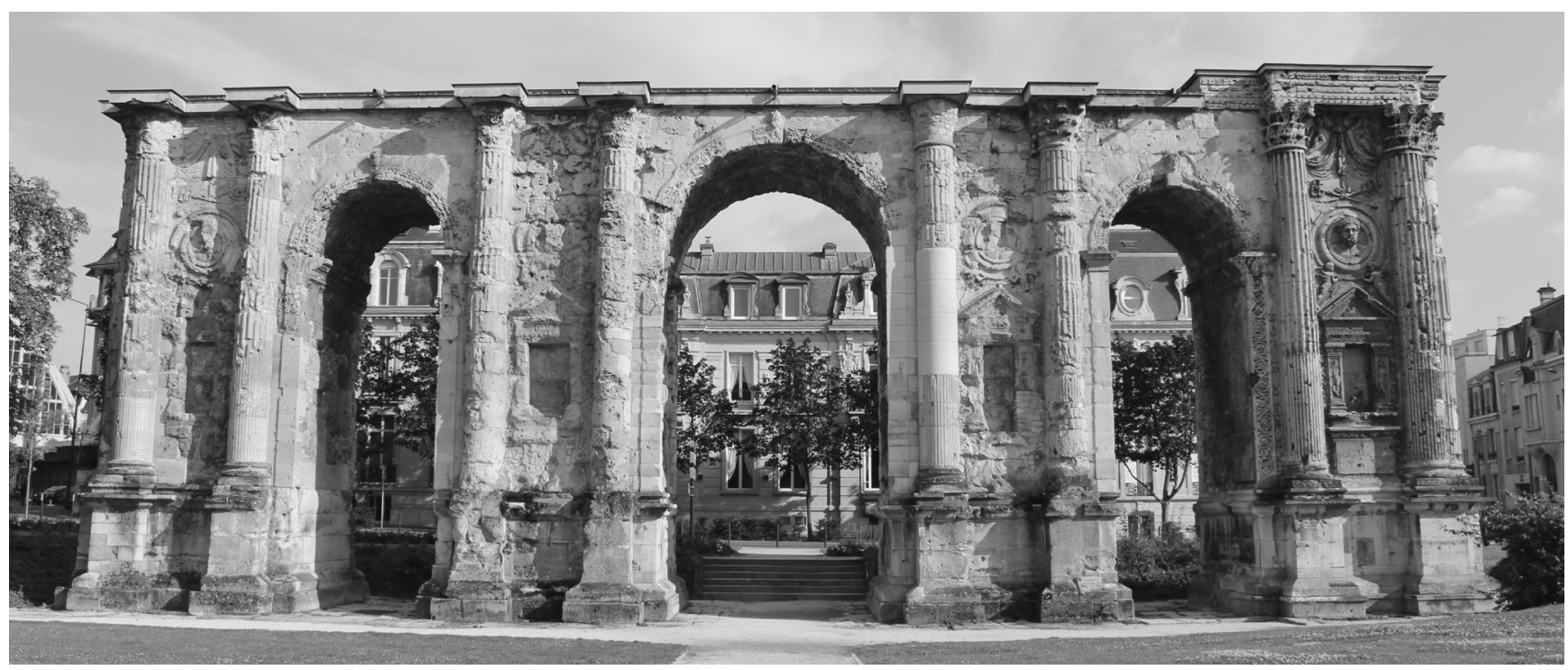

Figure 44 : La façade septentrionale de la porte de Mars à Reims. Cliché Yvan Maligorne.

Figure 44: The northern side of the Porte de Mars, in Reims.

et nous ne pouvons rien dire du contexte dans lequel il évoluait.

L'homme nu marchant à grande enjambée (fig. 42e) qui décorait le panneau d'une pile ne saurait lui non plus susciter des commentaires trop précis : la nudité quasi-totale, seulement tempérée par une probable draperie, et la marche vigoureuse ne sont pas incompatibles avec Bacchus, qui est représenté dans des attitudes voisines sur bien des images, comme sur l'emblema d'une mosaïque de Langres (Darmon, 1999; fig. 45), mais ce n'est là qu'une des solutions possibles.

Ce n'est en revanche pas l'hypothèse dionysiaque que nous privilégions pour les masques suspendus à des branches (fig. 42f) : comme l'a souligné Henri Lavagne (1987), les masques et têtes qui relèvent assurément de cette thématique sont suspendus à des rubans dessinant des réseaux réguliers à la surface de colonnes; l'exemple de Bayeux relève d'un autre type et ces masques suspendus à des branches rappellent de près des figurations de Lutèce et Trèves que le savant rapproche du culte métroaque (ibid., p. 42-44 et 47-51). Le couvre-chef de Bayeux est différent du bonnet phrygien de ces reliefs, ce en quoi la référence à Mithra ne nous semble pas assurée dans le cas qui nous occupe - elle serait d'ailleurs difficile à expliquer.

Enfin, deux reliefs prouvent que Bacchus et son thiase ne constituent pas les sujets uniques du décor du monument : Triton (fig. 42g) et la figure guerrière de la colonne (fig. 17a) n'entretiennent pas de rapports explicites avec cet univers. D'ailleurs, nombreux sont les exemples gaulois qui montrent, en particulier la Porte Noire de Besançon, que l'iconographie d'un même monument peut mobiliser au

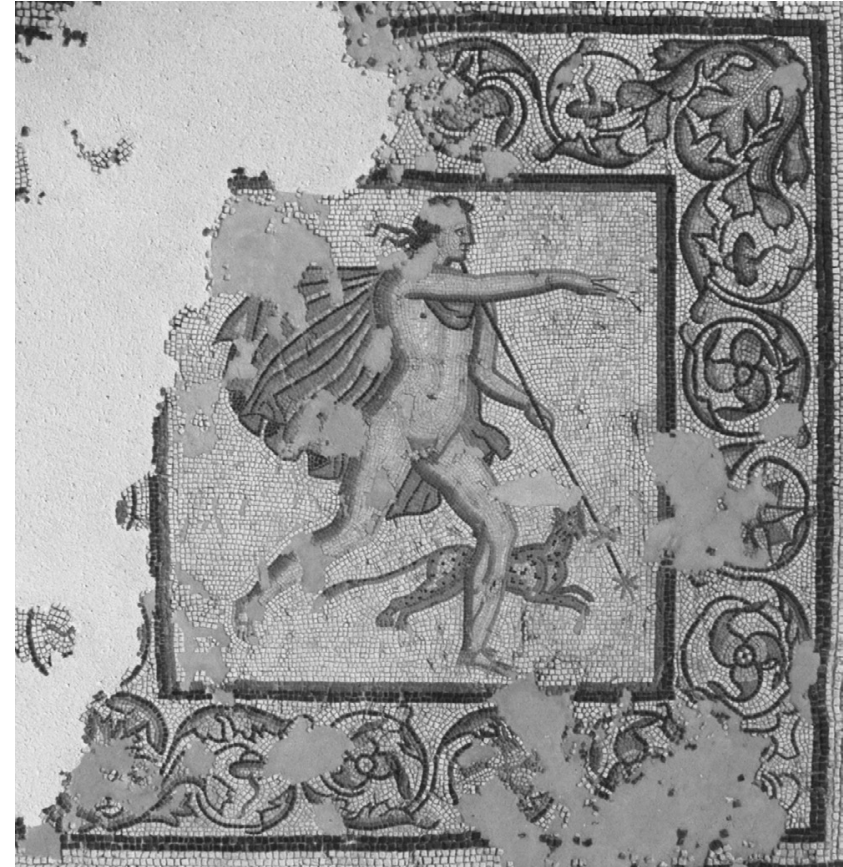

Figure 45 : Emblema d'une mosaïque de Langres figurant Dionysos. Cliché Arnaud Vaillant, musées de Langres.

Figure 45 : Emblema from a mosaic in Langres picturing Dionysos.

service d'un discours cohérent des éléments empruntés à des sphères très différentes.

À en juger par les éléments qui nous sont parvenus, le monde de Bacchus est bien représenté, comme d'ailleurs sur de nombreux monuments gaulois des $\mathrm{II}^{\mathrm{e}}-\mathrm{III}^{\mathrm{e}}$ siècles relevant 
de types très variés. Il n'est guère de catégorie monumentale qui échappe à ce goût pour l'iconographie dionysiaque. Dans l'ouest, il faut citer le sanctuaire de Mars Mullo à Allonnes (Gury, 2004), mais aussi des maisons : la domus de Vieux déjà évoquée (Vipard, 1997, p. 218-228 et 291-297; 1998, p. $76-85$; 2001) et la villa du Mané-Véchen à Plouhinec, sur le littoral des Vénètes, dont le décor développe de nombreuses références dionysiaques, sous forme de peintures, reliefs stuqués - dont une véritable mégalographie - et relief calcaire figurant la découverte d'Ariane à Naxos (Provost, 2007, p. 97-98 et 100; Boislève, Provost, 2011).

Cette récurrence inspire deux lectures opposées. Les symboles bachiques qui s'accumulent sur les colonnes et les entablements se voient parfois refuser toute signification réelle et sont ravalés au rang de manifestations d'une mode ornementale (Tardy, 2005, p. 126; voir aussi supra, n²6). À l'inverse, sont parfois proposées des interprétations maximalistes, des chercheurs identifiant des bâtiments dont le décor est dominé par une ambiance bachique à des sièges d'associations dionysiaques (Lavagne, 1986, p. 145; 1987, p. 43, avec $\left.n^{\circ} 34\right)$. La difficulté - et nous devons cette remarque en forme d'avertissement à Stéphanie Wyler, spécialiste de l'iconographie dionysiaque - réside dans le fait que le dieu est partout à sa place et que sa présence peut revêtir des sens très différents. Il est peut-être excessif de refuser toute signification à l'accumulation de ses symboles dans le décor d'architecture, ne serait-ce que parce qu'ils contribuent à créer une atmosphère, fût-ce sur un mode allusif. Dans les maisons, ils évoquent ce monde de la fête, des plaisirs et de l'abondance auquel Paul Zanker a consacré de belles pages, et se rattachent en cela à une tradition qui remonte à l'époque tardo-classique ${ }^{30}$. Ce type de référence suffit le plus souvent à expliquer l'abondance des thèmes dionysiaques, sans qu'il soit besoin d'en appeler à une hypothétique fonction de sièges d'association.

Mais Dionysos-Bacchus est aussi un héros victorieux et civilisateur qui triomphe de tous ses adversaires; c'est cette dimension que met en avant Françoise Gury pour expliquer sa présence dans le décor du sanctuaire de Mars Mullo à Allonnes (Gury, 2004). On peut se demander si le personnage, Hercule ou Mars (?), figuré sur la colonne engagée de Bayeux (blocs 5 et 6 ) ne participe pas de cette thématique triomphale, de cette évocation de la virtus et de la victoria; dans le même ordre d'idées, on ne manquera pas de

30. Zanker 2001, en part. p. 62-65; Zanker, Ewald, 2008, p. 150-152. Stéphanie Wyler a cependant montré qu'on ne saurait réduire à un sens unique l'évocation de l'univers dionysiaque dans les maisons de l'époque tardo-hellénistique et du début de l'époque impériale (Wyler, 2004). Dans les maisons italiennes de la fin de l'époque républicaine, le recours à ces décors traduit un philhellénisme et surtout un attrait pour les royautés hellénistiques dont on peut douter qu'ils aient joué le moindre rôle dans les maisons et monuments gaulois des $\mathrm{II}^{\mathrm{e}}$ et $\mathrm{III}^{\mathrm{e}}$ siècles. remarquer que Triton et autres créatures marines peuvent évoquer la domination universelle de l'empereur, sur la terre et les mers, terra marique nous disent les inscriptions et les légendes monétaires ${ }^{31}$. Le triton acquiert cette signification après Actium (Hölscher, 1985), et c'est à ce titre que le trophée augustéen de Saint-Bertrand-de-Comminges lui fait une place (Boube, 1996, p. 27 et 44). Sans appartenir au répertoire le plus courant des arcs, il apparait sur un arc augustéen d'Arles (Fornasier, 2003, p. 46-47, 158159 pl. VIII-2 et IX-1); les centaures marins qui figurent sur les arcs d'Orange et de Die auraient été chargés de la même signification (ibid., p. 159). Le bouclier suspendu à des ténies possède quant à lui une probable valeur apotropaïque (Walter, Bruchet, 1986, p. 400-401).

Cependant, ce qui est conservé du décor de l'arc de Bayeux renvoie moins explicitement au thème de la victoire qu'à ceux, connexes, de l'abundantia, de la fecunditas et de la felicitas temporum, qui jouent un rôle central dans l'idéologie impériale. Les pilastres, colonnes et archivoltes du monument de Bayeux déclinent ce discours avec leurs anthémions et rinceaux d'essences multiples; il était sans nul doute conforté par les chapiteaux et un entablement lourde-

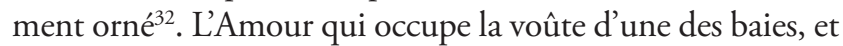
dont nous n'avons pu déterminer dans quelle action il était engagé, participe peut-être de l'évocation d'un monde de fête et de bonheur - on retrouve des Erotes en pareille position sur l'arc de Besançon (Walter, Bruchet, 1986, p. 168171, 405, pl. LXVII-LXIX et fig. 46) et sur l'intrados d'un petit arc découvert à Lillebonne ${ }^{33}$.

L'interprétation du décor ne peut pas être univoque et autorise différents niveaux de lecture, d'autant, répétons-le, que la plus grande partie nous en est inconnue. Les choix iconographiques opérés par les concepteurs du monument de Bayeux ne sont pas sans parallèles, et l'on remarque avec intérêt que plusieurs arcs déploient des thématiques voisines : outre la Porte Noire de Besançon, plusieurs fois citée,

31. Plusieurs reliefs du $\mathrm{II}^{\mathrm{e}}$ siècle figurent des arcs érigés dans des ports : ils sont dominés par une statue de Neptune dressée sur l'attique et comportent des images de tritons, qui évoquent le royaume marin du dieu (Kleiner, 1991, p. 213-216). Ce qui est conservé du relief de Bayeux ne permet pas de déterminer s'il faut s'en tenir à un sens très générique ou postuler un rapport plus précis avec le pouvoir universel de l'empereur.

32. Ce discours figuratif sur l'abondance est repris à l'identique sur des monuments funéraires, par exemple sur le monument de Saintes que nous avons déjà invoqué à titre de comparaison (fig. 28) : voir Maurin 1978, p. 129 et fig. 180-181 (p. 415). Pour la signification des thèmes dionysiaques dans l'iconographie funéraire, voir Zanker, Ewald, 2008, p. $135-149$ et $156-167$.

33. Espérandieu, IV, 3109. Seul est conservé le départ de l'arc, qui jouxte un pilastre orné d'un personnage nu ressemblant étrangement à celui de notre bloc 20, mais qui ne livre malheureusement aucun élément positif d'identification. Nous devons donc nous contenter de souligner les parentés entre ce bloc et certaines des composantes du répertoire mis en œuvre à Bayeux. 


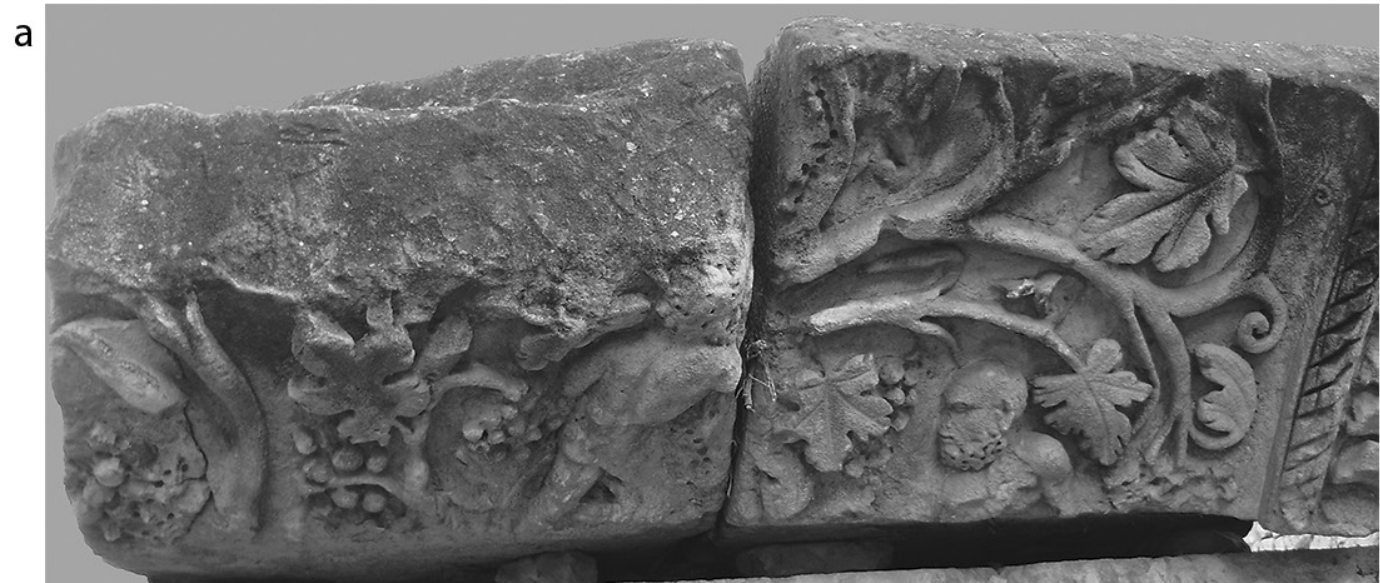

b

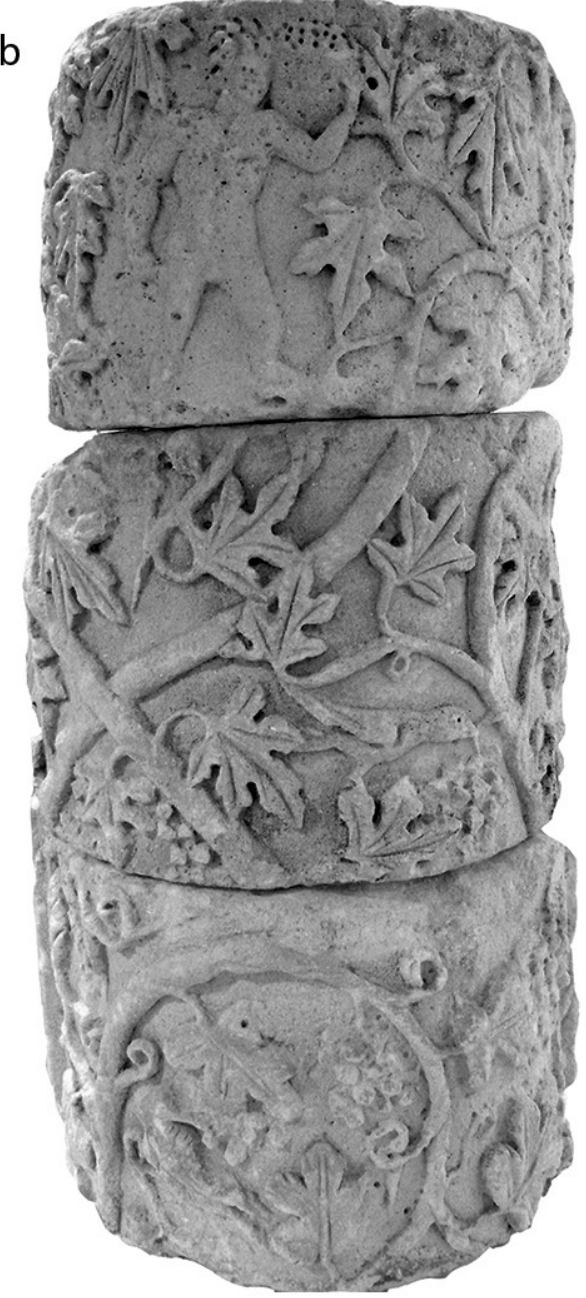

Figure 46 : Vestiges d'un arc d'Arles. (a) Intrados de la voûte orné d'un Silène (?) dans une vigne (théâtre d'Arles). (b) Tambours de colonne décorée d'un rinceau de vigne (musée départemental de l'Arles antique). Clichés Yvan Maligorne.

Figure 46: Remains of an arch in Arles. (a) Lower side of the vault decorated with a Silenus (?) in a vine (Arles theatre). (b) Column decorated with a vine scroll (musée départemental de l'Arles antique). il faut signaler les vestiges d'un arc d'Arles (Küpper-Böhm, 1996, p. 146-152; Bartette, 2013, vol. 1, p. 129, vol. 2, p. 24-25 et 155, vol. 3, pl. 20 et 189). Ce dernier monument juxtapose symboles guerriers (amas d'armes) et évocation de la prospérité (colonnes ornées de rinceaux de vigne et claveaux dont l'intrados est orné d'une vigne luxuriante peuplée d'un probable Silène; fig. 46). L'abondance et la prospérité, corollaires de la victoire impériale, ne sont certes pas des nouveautés de la période antonine : depuis le début de la période impériale, arcs et portes accueillent fréquemment - c'est particulièrement vrai dans les Gaules - une décoration végétale foisonnante, qui habille en particulier pilastres et archivolte, donc les éléments qui encadrent et soulignent les passages. Mais ce discours revêt désormais des formes plastiques différentes, gagne des emplacements nouveaux, moins restreints; surtout, alors que l'univers dionysiaque a longtemps souffert d'un statut ambigu qui l'a empêché de s'épanouir pleinement dans la sphère publique ${ }^{34}$, ces réserves sont levées dès l'orée du $\mathrm{II}^{\mathrm{e}}$ siècle, comme en témoigne éloquemment le décor de la cella du temple de Vénus Genetrix, sur le forum de César, dans sa réfection domitiano-trajanienne (Milella, 2010, p. 460-464).

\section{Conclusion}

Les collections lapidaires de Bayeux sont riches de plusieurs dizaines de blocs. La plupart, cependant - bases attiques, fûts lisses et chapiteaux toscans - sont des éléments isolés, complètement coupés de leur contexte architectural premier, et n'offrent de surcroît aucun élément de datation intrinsèque. L'ensemble examiné dans cet article se distingue par sa valeur documentaire : cohérent sur le plan stylistique,

34. Zanker, Ewald 2008, p. 153-154; voir les précisions apportées par Wyler, 2013 sur la place accordée à Dionysos dans la sphère privée dès l'époque augustéenne, et ce, dans l'entourage même du Prince. 
il est dominé par des séries de claveaux qui permettent une identification assurée d'un type de monument très mal documenté en Gaule de l'Ouest.

De fait, il est difficile de déterminer la place exacte de l'arc monumental dans l'urbanisme des Gaules aux II ${ }^{\mathrm{e}}$-III ${ }^{\mathrm{e}}$ siècles. À en juger par les inventaires disponibles (Kähler, 1939, col. 414-423; De Maria, 1994; Fornasier, 2003; Blonce, 2008), Trois Gaules et Germanies n’ont rien livré qui soit comparable à la série sud-gallique de la période augusto-tibérienne, encore moins aux monuments africains des périodes antonine et sévérienne. Certes, ces inventaires peuvent être ponctuellement révisés et complétés - cette contribution en est l'illustration -, mais cela ne bouleverse pas l'évaluation du poids de ce type monumental dans l'urbanisme des provinces nord-occidentales de l'Empire.

En Gaule du Nord-Ouest, rares sont les arcs monumentaux. Les fouilles de la rue Saint-Malo, à Rennes, ont livré deux fosses quadrangulaires symétriquement disposées de part et d'autre d'une voie; elles révèleraient selon G. Le Cloirec la spoliation totale d'un arc dont les données stratigraphiques datent la construction postérieurement au milieu du $\mathrm{II}^{\mathrm{e}}$ siècle $^{35}$. Les deux massifs rectangulaires accolés à la façade de la basilique de Vannes appellent la restitution d'un passage voûté, mais l'évocation graphique proposée par P. André et reprise par B. Fornasier ne peut s'appuyer sur le moindre élément d'architecture (Triste et André, 1992, p. 95, restitutions graphique et plastique p. 31-33; Fornasier, 2003, p. 142-144). Enfin, plusieurs blocs d'architecture découverts à Avranches ont été interprétés par le chanoine Pigeon, à la fin du XIX ${ }^{\mathrm{e}}$ siècle, comme les vestiges d'un arc; la série lapidaire, modeste, ne comportait que quatre blocs, dont l'un portait sur deux faces adjacentes deux colonnes engagées; selon l'érudit, la plus petite aurait supporté la retombée d'une voûte et la plus grosse aurait scandé la façade d'un arc auquel il restitue trois baies (fig. 47-48) (Archives municipales d'Avranches, Fonds Pigeon, Ms 94, p. 39 et 42; Levalet, 2010, p. 140 avec fig. 146). Mais un tel bloc n'impose nullement la restitution d'une baie voûtée, encore moins d'un arc monumental : les archivoltes des arcs retombent sur des pilastres et non sur des colonnes engagées. De surcroît, les piles du monument dessiné par le chanoine sont entièrement occupées par une colonne engagée, ce qui est curieux. Enfin, le bloc attribué à l'entablement développe une singulière séquence moulurée qui interdit de lui assigner

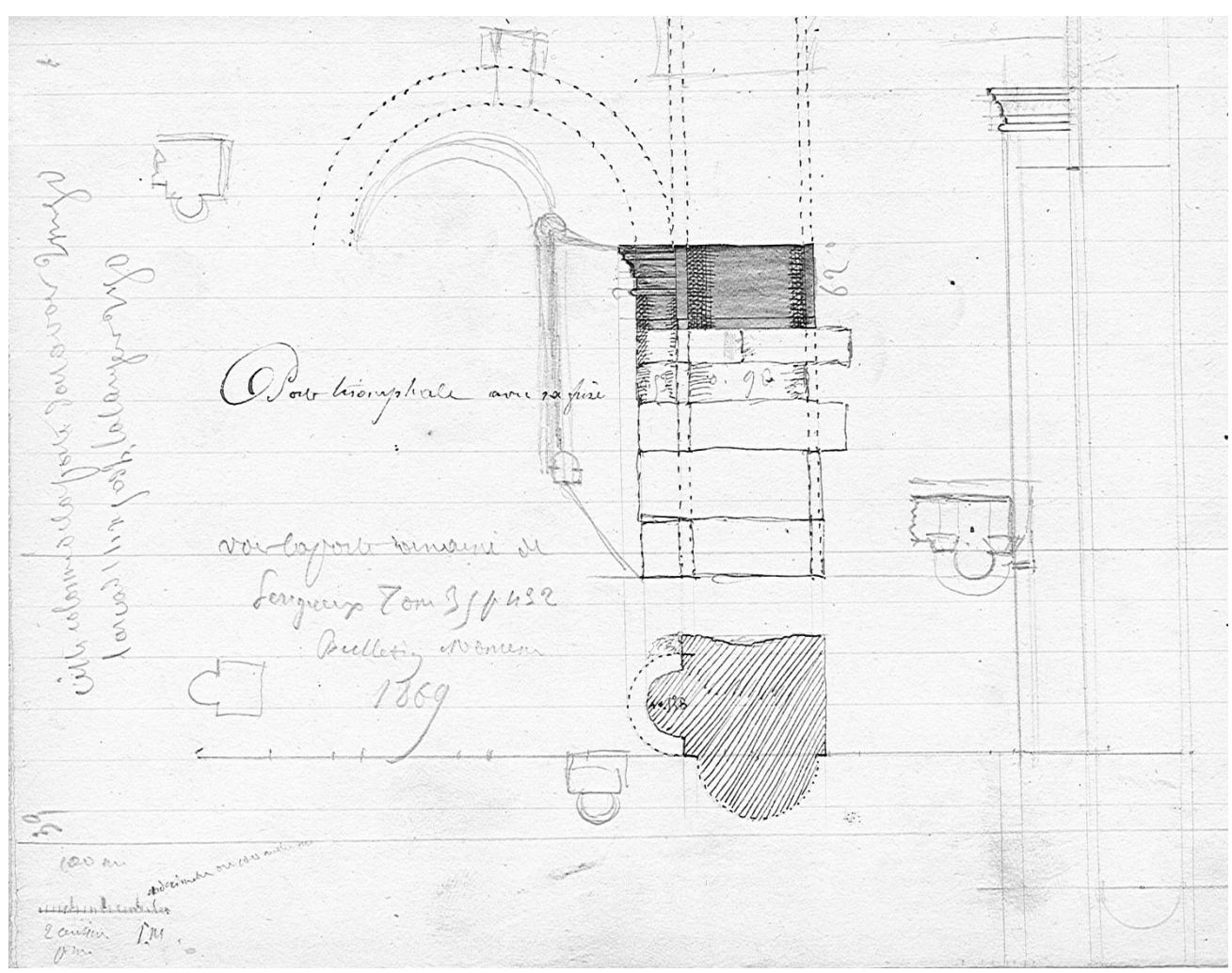

Figure 47 : Croquis d'un bloc découvert à Avranches. Avranches, archives départementales, fonds Pigeon, Ms 94, p. 39.

Figure 47: sketch of a block discovered at Avranches.

35. Le Cloirec, 2008, p. 274-276 et 280-282. Les fosses de récupération des piles mesuraient 3,60 × 3 mètres et la baie était large de 4,80 mètres; le monument était donc long de 12 mètres. L’arc était pleinement urbain, sauf à postuler une très forte rétraction de la ville à cette époque. 


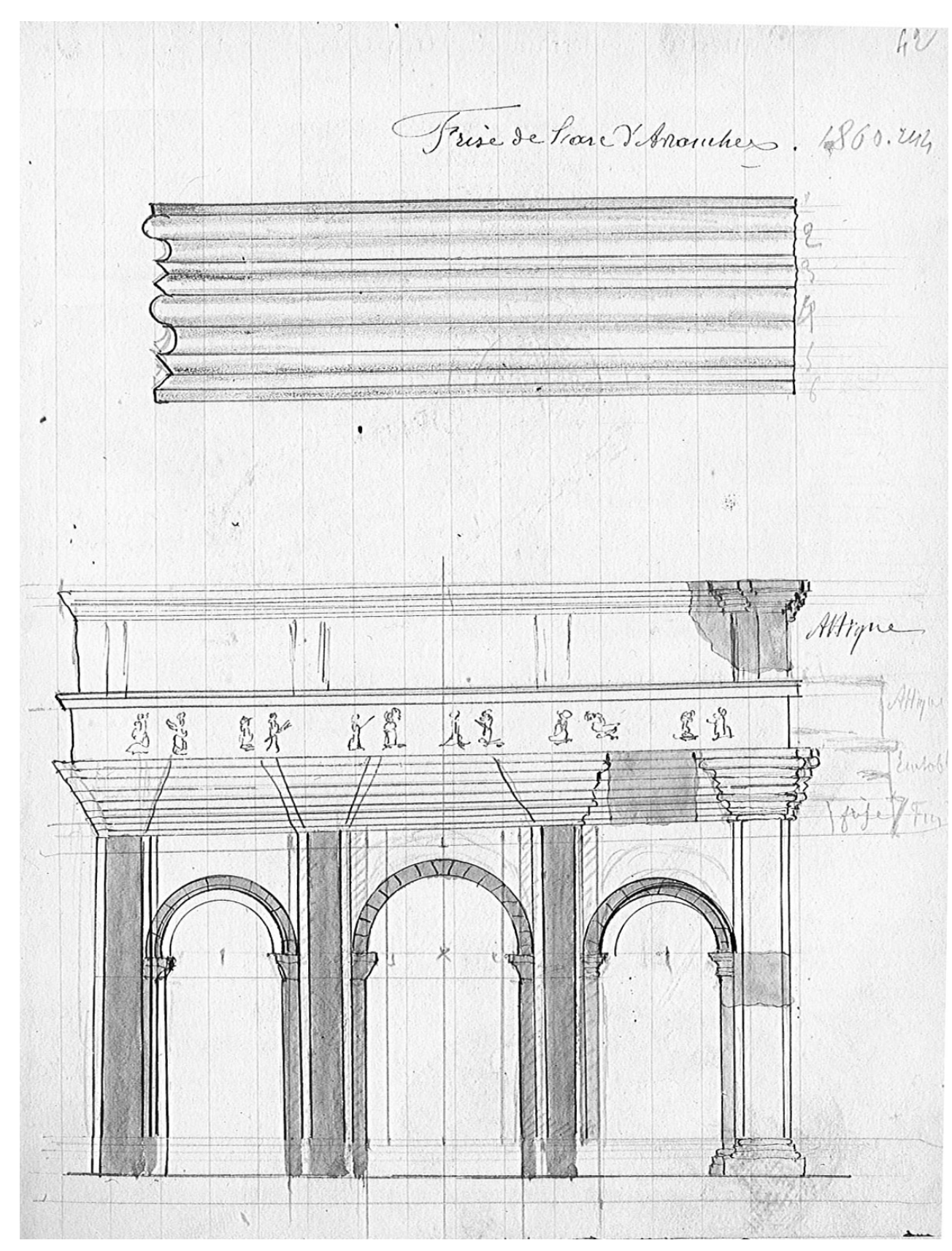

Figure 48 : Relevé d'un bloc et restitution hypothétique d'un arc. Avranches, archives départementales, fonds Pigeon, Ms 94, p. 42.

Figure 48: Drawing of a block and restitution of an arch.

pareille position et amène même à douter de son antiquité. Ce monument doit donc être écarté, et le dossier régional est des plus minces.

L'enquête que nous avons conduite n'a pu répondre à toutes les questions que soulève un tel édifice. Sa localisation dans le tissu urbain est inconnue, ce qui nous laisse dans l'ignorance des rapports qu'il ne manquait pas d'entretenir avec d'autres monuments, avec un axe important du réseau viaire ou avec un espace découvert. Aucun élément ne permet de penser que l'arc s'élevait à proximité immédiate du lieu de découverte des blocs : si le secteur accueille des vestiges gallo-romains, aucun ne renvoie avec certitude à un édifice public. Quant à l'hypothèse selon laquelle la cathédrale recouvrirait un sanctuaire antique, elle n'est étayée par aucun argument contraignant; la présence de mortier sur le décor de certains blocs montre qu'ils ont été remployés et que tel n'était pas leur emplacement primitif ${ }^{36}$.

Sa datation, au début du $\mathrm{III}^{\mathrm{e}}$ siècle, ne nous apprend rien de concret sur les raisons de sa construction. Rappelons que tous les arcs n'étaient pas érigés pour l'empereur régnant ou

36. Quant aux édifices publics (ou supposés tels) connus à proximité du lieu de découverte des blocs, qu'il s'agisse de l'ensemble thermal de la rue Laitière ou de la place publique du 10 rue Franche, ils sont trop éloignés pour qu'on puisse envisager leur extension jusqu'à la cathédrale. 
un divus : certains étaient dédiés à des particuliers, d'autres à des dieux immortels - les arcs de Genève et de Mayence, dédiés à IOM, en offrent des exemples clairs en contexte gaulois (Kleiner, 1991, p. 216-217; 1992, p. 167). Le décor figuré du monument de Bayeux semble cependant, pour autant que nous puissions en juger, renvoyer à des thèmes de l'idéologie impériale, comme l'abondance et la felicitas temporum. Il ne commémorait pas nécessairement une victoire précise, encore moins un triomphe particulier : il était peutêtre tout simplement conçu pour rappeler que les victoires de l'empereur sont le fondement de la prospérité de l'Empire et du bonheur des citoyens.

\section{Remerciements}

Les recherches présentées dans cet article ont été exposées en mars 2013 à l'occasion de la Huitième journée d'étude sur l'ouest de la Gaule romaine, à Brest; nous avons alors bénéficié de remarques des professeurs Patrick Le Roux et Valérie Huet. Cette étude doit beaucoup à M. Antoine Verney, conservateur en chef des musées de Bayeux, et à l'équipe du Musée d'Art et d'Histoire de Bayeux (MAHB) : ils nous ont permis de travailler dans d'excellentes conditions sur un mobilier qui pose des problèmes de manutention, lesquels peuvent en entraver l'étude. Xavier Savary, géologue au service Archéologie du conseil général du Calvados, nous a permis d'utiliser les résultats de son étude pétrographique. Au moment d'affronter la question du dessin d'un monument si incomplètement conservé, nous nous sommes efforcés de garder à l'esprit les réflexions toujours stimulantes d'Alain Badie (Institut de recherche sur l'architecture antique, CNRS) sur les restitutions architecturales et ce qu'on est en droit de leur demander. Nous devons à M. Arnaud Vaillant, attaché de conservation aux musées de Langres, le cliché de la mosaïque dionysiaque de Langres, et à $M$. Daniel Levalet, vice-président de la Société archéologique d'Avranches, la reproduction des dessins du chanoine Pigeon. Le musée des Antiquités de Rouen nous a généreusement confié un cliché de la stèle de Lillebonne. Que tous soient ici chaleureusement remerciés.

\section{Bibliographie}

\section{Sources}

Grégoire de Tours, Histoire des Francs, Paris, 1995, 3e tirage 2005 (trad. Robert Latouche).

\section{Études}

Adam J.-P., Deyts S. et Saulnier-Pertuit L., i 987 - La façade des thermes de Sens, $7^{\mathrm{e}}$ suppl. à la Revue archéologique de l'Est et du Centre-Est, Dijon, 48 p. +29 pl.
BARTETTE T., 2OI 3 - Le décor architectonique de l'Arles antique (thèse de doctorat, université d'Aix-Marseille), 3 vol., VIII + 277 p., VI + 206 p., XII + 189 pl.

BézIers M., I773 - Histoire sommaire de la ville de Bayeux, Caen, J. Manoury, 376 p.

Blanc N. et Gury F., 1986 - "Eros, Amor, Cupido", in Lexicon Iconographicum mythologiae classicae, III, Atherion-Eros, Zurich, Munich, 1986, vol. 1, p. 952-1049, vol. 2, p. 678-727.

BLONCE C., 2008 - L'arc monumental dans le monde romain, du début du It siècle au début du IV siècle apr. J.-C. : histoire et place dans la vie politique, religieuse et sociale (thèse de doctorat, université de Paris IV-Sorbonne), 3 vol., $574+928+498$ p.

Boislève J. et Provost A., 20 I I - « Les stucs de la villa maritima de Mané Véchen, anciennes découvertes et nouveaux décors ", in Balmelle C., Eristov H. et Monier F., Décor et architecture en Gaule entre l'Antiquité et le haut Moyen Âge : mosä̈que, peinture, stuc, actes du colloque de Toulouse, Aquitania, suppl. 20, Bordeaux, p. 539-552.

Bossert M., 1998 - Die figülichen Baureliefs des CigognierHeiligtums in Avenches. Kunsthistorische und ikonologische Einordnung, Lausanne (Aventicum VIII, Cahiers d'archéologie romande), 187 p., 47 p. de pl.

Boube E., 1996 - Collections du musée archéologique départemental de Saint-Bertrand-de-Comminges, 4. Le trophée augustéen, Saint-Bertrand-de-Comminges, Saint-Bertrand-de-Comminges, Musée archéologique départemental, $160 \mathrm{p}$.

Bourdon C., I 85 I - "Excursion archéologique à la cathédrale de Bayeux ", Bulletin Monumental, $2^{\mathrm{e}}$ série, tome 7, $17^{\mathrm{e}}$ vol., Paris, p. 196-214.

Bourrienne V., I902-1903 - Antiquus cartularius ecclesiae baiocensis (livre noir), Paris, Rouen, Picard, 471 p.

Caumont A. (DE), I857-Statistique monumentale du Calvados : arrondissements de Vire et de Bayeux, tome II, Caen, A. Hardel, $808 \mathrm{p}$.

DalibaRd M., I 977 - Ville de Bayeux, recherche du tracé romain, évolution historique (mémoire de diplôme d'architecte dactylographié), Caen, $114 \mathrm{p}$.

Darmon J.-P., I999 - « Dionysos chez les Lingons. Le pur classicisme de la mosaïque de Langres ", in Blanc N. et Buisson A. (éd.), Imago antiquitatis. Religions et iconographie du monde romain. Mélanges offerts à Robert Turcan, Paris, de Boccard, p. 197-207.

Delacampagne F., I 986 - Bayeux (Calvados), 12 rue Laitière, rapport de sauvetage urgent, Caen, Service archéologie, Conseil général du Calvados, $16 \mathrm{p}$.

Delacampagne F., 1987 - Bayeux (Calvados), 12 rue Laitière, rapport de sauvetage urgent, Caen, Service archéologie, Conseil général du Calvados, $36 \mathrm{p}$.

Delacampagne F., i990a - Bayeux (Calvados), bas-côté nord de la Cathédrale, rapport de sauvetage urgent, Caen, Service archéologie, conseil général du Calvados, 22 p. 
Delacampagne F., I 990b - Le Calvados. Carte archéologique de la Gaule, Paris, éditions de la Maison des sciences de l'homme, 166 p.

Delacampagne F., I 997 - "Les thermes de la rue Laitière à Bayeux (Calvados). Réflexions sur la topographie antique et médiévale d'un quartier de la ville ", Revue archéologique de l'Ouest, 14, p. 125-173.

Delacampagne F., I $997 \mathrm{~b}$ - « Note à propos d'un bâtiment trouvé sous la cathédrale de Bayeux ", Bulletin de la Société des sciences, arts et belles-lettres de Bayeux, 31, p. 8-11.

Delacampagne F., 2003 - Bayeux (Calvados). Bas-côté sud de la cathédrale, rapport de diagnostic archéologique, Caen, Service archéologie, Conseil général du Calvados, 24 p.

Delacampagne F., 2007 - Bayeux (Calvados), chevet de la cathédrale, rapport de fouille programmée, Caen, Service archéologie, Conseil général du Calvados, 58 p.

Delacampagne F. et Paillard D., I 992 - Bayeux, documents d'évaluation du patrimoine archéologique des villes de France, Tours, $90 \mathrm{p}$.

Delplace C., I980 - Le griffon de l'archaïsme à l'époque impériale. Étude iconographique et essai d'interprétation symbolique, Bruxelles, Institut historique belge de Rome, 460 p.

De Maria S., I 994 - "Arco onorario e trionfale ", Enciclopedia dell'arte antica classica e orientale, supplemento 1971-1994, Rome, Istituto della enciclopedia italiana Treccani, vol. I, p. 354-377.

Dion H. De et Lasvignes L., i 86 I - Cathédrale de Bayeux. Reprise en sous-ceuvre de la tour centrale, Paris, A. Morel, 104 p., pl.

Doucet L., I88I-I882 - «Les thermes de la rue Laitière à Bayeux, lettre de M. Doucet à M. le secrétaire des Antiquaires de Normandie ", Bulletin de la Société des Antiquaires de Normandie, XI, p. 444-445.

Doucet L., I 882 - "Rapport sur les fouilles nécessitées pour la construction d'un Hôtel des Postes rue Laitière ", L'Écho Bayeusain, $\mathrm{n}^{\circ}$ du 8 décembre, Bayeux, p. 1-10.

EspérANDIEU É., I907-I949 - Recueil général des bas-reliefs, statues et bustes de la Gaule romaine, I-XIII, Paris, Imprimerie nationale.

Farcy P. DE, 1879 - "Bayeux en 1780 ", Mémoires de la Société d'Agriculture, des Sciences, Arts et Belles lettres de Bayeux, IX, p. 207.

Follain E., Langlois J.-Y. et Bocard H., I 996 - Rouen. Les fontaines de la place de la Pucelle. III ${ }^{e}$ XVI et XVIII siècles, Itinéraires du Patrimoine 105, Paris, Service régional de l'Inventaire, 17 p.

ForNAsIER B., 2003-Les fragments architecturaux des arcs triomphaux en Gaule romaine, Annales littéraires de l'université de Franche-Comté, 746, Besançon, 304 p.

Frenz H.G., I98 I - «Der Ehrenbogen des Dativius Victor zu Mainz und seine neue Rekonstruktion ", Bericht der RömischGermanischen Kommission, 62, p. 219-260, Taf. 34-44, Bei. $9-11$.
Gaggadis-Robin V., 2005 - Les sarcophages pä̈ens du musée de l'Arles antique, Cahors, éditions du musée de l'Arles et de la Provence antiques, $332 \mathrm{p}$.

Gauthier N. et Fixoт M., I 996 - Topographie chrétienne des cités de la Gaule des origines au milieu du VII' s. IX, Province ecclésiastique de Rouen (Lugdunensis Secunda), Paris, de Boccard, 95 p.

Gros P., 2011 - L'architecture romaine, 1, Les monuments publics, Les manuels d'art et d'archéologie antiques, Paris, Picard, $3^{\mathrm{e}}$ édition, $503 \mathrm{p}$.

Gury F., 2004 - "L'iconographie religieuse ", in Gruel K. et Brouquier-Reddé V. (dir.), « Le sanctuaire de Mars Mullo chez les Aulerques Cénomans (Allonnes, Sarthe). ve siècle av. J.-C. - Iv siècle apr. J.-C. », Gallia, 61, p. 354-360.

Heuzey L., I 869 - "Le dieu Mên à Bayeux ", Revue archéologique, 19 , p. $1-6$, pl. 1.

Hölscher T., I 985 - «Denkmäler der Schlacht von Actium. Propaganda und Rezonanz ", Klio, 67, 1, p. 81-102.

Huard G., I9I7 - "Chronique archéologique. Année 1917 », Bulletin de la Société des Antiquaires de Normandie, p. 367.

ICARD-GIAnOLO N., I997A - " Triton ", in Lexicon iconographicum mythologiae classicae, VIII, Thespiades-Zodiacus et supplementum Abila-Thersites, Zürich-Düsseldorf, vol. 1, p. 68-73, vol. 2, p. $42-46$.

ICARD-GIAnOlO N., I997B - "Tritones ", in Lexicon iconographicum mythologiae classicae, VIII, Thespiades-Zodiacus et supplementum Abila-Thersites, Zürich-Düsseldorf, vol. 1, p. 73-85, vol. 2, p. 46-60.

KäHlER H., I 939 - s.v. "Triumphbogen (Ehrenbogen) ", in Realencyclopädie der classischen Altertumswissenschaft, VII, A1, col. 373-493.

Kleiner F.S., I99 I - "The sanctuary of the Matronae Aufaniae in Bonn and the tradition of votive arches in the Roman world", Bonner Jahrbücher, 191, p. 199-224.

Kleiner F.S., I 992 - "The trajanic gateway to the Capitoline sanctuary of Jupiter Optimus Maximus ", Jahrbuch des Deutschen Archäologischen Instituts, 107, 149-174.

Küpper-Böнm A., 1996 - Die römischen Bogenmonumente der Gallia Narbonensis in ihrem urbanen Kontext. Espelkamp, Marie Leidorf, XIII + 238 p.

Lambert C.-É., I 824 - « Premier et deuxième mémoires sur les thermes antiques dans l'ancien cimetière Saint-Laurent de la ville de Bayeux ", Mémoires de la Société des Antiquaires de Normandie, première partie, p. 17-49.

Lambert C.-É., I 825 - "Troisième mémoire sur les thermes antiques dans l'ancien cimetière Saint-Laurent de la ville de Bayeux ", Mémoires de la Société des Antiquaires de Normandie, p. $146-156$.

LAMBERT C.-É., I 829-I 830 - « Extrait d'une lettre adressée à M. de Caumont sur quelques débris romains exhumés à Bayeux près de la Cathédrale ", Mémoires de la Société des Antiquaires de Normandie, p. 331-335, Atlas, fig. 2, pl. XII. 
Lavagne H., I986 - "Rome et les associations dionysiaques en Gaule (Vienne et Nîmes) ", in L'association dionysiaque dans les sociétés anciennes, actes de la table ronde organisée par l'École française de Rome (Rome, 24-25 mai 1984), Paris, Rome, coll. "École française de Rome; 89 ", p. 129-148.

LAVAGNE H., I987 - "Lutèce : le monument funéraire aux amours de Mars et les reliefs aux têtes d'Attis ", Les Cahiers de la Rotonde, 10, p. 37-67.

Le Cloirec G., 2006 - Bayeux (Calvados). Hôtel du Doyen (parcelles AI 77 et 80), rapport de diagnostic archéologique, Rennes, Service régional de l'archéologie, $104 \mathrm{p}$.

Le Cloirec G., 2008 - "La fouille du 3-5 rue Saint-Malo », in Pouille D. (dir.), Rennes antique, Rennes, p. 251-287 et pl. XXXIV-XXXIX.

Lefèvre F., I985 - La porte de Mars de Reims, Reims, Groupe d'études archéologiques Champagne-Ardenne, $60 \mathrm{p}$.

Levalet D., 2010 - Avranches et la cité des Abrincates. Ir siècle av. J.-C. - vII siècle apr. J.-C. (Revue d'histoire et d'archéologie, Mémoire de la Société des antiquaires de Normandie, XLV), 262 p.

LORILlu, I 89I - "Recherche sur les changements topographiques de Bayeux vers le pont de la Madeleine ", Mémoires de la Société des sciences arts et belles-lettres de Bayeux, I, p. 14-16.

Maligorne Y., 2006 - L'architecture romaine dans l'ouest de la Gaule, Rennes, Archéologie et culture, 229 p.

MatheA-FöRTsch M., I 999 - Römische Rankenpfeiler und-pilaster. Schmuckstützen mit vegetabilem Dekor, vornehmlich aus Italien und den westlichen Provinzen, Mayence, Deutsches archäologisches Instituts (Beiträge zur Erschließung hellenistischer und kaiserzeitlicher Skulptur und Architektur 17), 221 p., $120 \mathrm{pl}$.

MAURIN L., I978 - Saintes antique des origines à la fin du vie siècle après Jésus-Christ, Saintes, Société d'archéologie et d'histoire de la Charente-Maritime, 528 p.

Milella M., 20 io - "La decorazione del tempio di Venere Genitrice ", Scienze dell'Antichità. Storia, archeologia, antropologia, 16, p. 455-469.

Mitard P.-H., I993 - Le sanctuaire gallo-romain de Genainville, Guiry-en-Vexin, Centre de recherches archéologiques du Vexin français, $449 \mathrm{p}$.

Numrich B., I997 - Die Architektur des römischen Grabdenkmäler aus Neumagen. Beiträge zur Chronologie und Typologie (Trierer Zeitschrift, Beiheft 22), Trèves, 333 p.

Pensabene P., I996 - «Programmi decorativi e architettura del tempio di Antonino e Faustina al Foro Romano ", Studi miscellanei, 29, p. 239-269.

Pluquet F., I 829 - Essai historique sur la ville de Bayeux et son arrondissement, Caen, T. Chalopin, $432 \mathrm{p}$.

PrieUr J., I 982 - «Les arcs monumentaux dans les Alpes occidentales : Aoste, Suse, Aix-les-Bains ", ANRW, II, Principat, 12, 1, Walter de Gruyter, Berlin, New York, p. 442-475.
Provost A., 2007 - "La "villa” maritime de Mané-Véchen à Plouhinec (Morbihan)", Aremorica. Études sur l'ouest de la Gaule romaine, 1, p. 85-100.

QUÉTIER A.-M., I98 I - La topographie d'Augustodurum, mémoire de maîtrise dactylographiée, Université de Caen, 143 p.

SAuvage R.-N., I909 - "La Basse-Normandie gallo-romaine (Lexovii, Viducasses, Baiocasses) ", in Congrès archéologique de France : LXXVe session tenue à Caen en 1908 par la Société française d'archéologie, tome II, Procès-verbaux et Mémoires, Paris, Caen, p. 506-507.

SAuvage R.-N., I9Io - La Basse-Normandie gallo-romaine (Lexovii, Viducasses, Baiocasses), Caen, H. Delesques, 16 p.

Schütz G., Sauvin Ch.-É., 20 I I - Bayeux (Calvados). 13, rue de Nesmond, Jardin des Augustines (parcelles AL 193a, 196 et 270), rapport final de diagnostic archéologique, Caen, Service archéologie, conseil général du Calvados, 2011, 133 p.

Schütz G., SAuvin C.-É., 20 I в - Bayeux (Calvados). 10 rue Franche, rapport final de fouille programmée, Caen, Service archéologie, Conseil général du Calvados, 361 p.

Schütz G., Sauvin C.-É., Borderie Q., Piolot A. et Thiesson J., 2013 - « Bayeux (Calvados) de l'Antiquité au haut Moyen Âge : approche interdisciplinaire des opérations récentes en centre-ville ", in Lorans E. et Rodier X. (dir.), Archéologie de l'espace urbain, Tours, p. 93-108.

SinN F., 1987 - Stadtrömische Marmorurnen, Beiträge zur Erschliessung hellenistischer und kaiserzeitlicher Skulptur und Architektur, VIII, Mayence, Philip von Zabern, X + 315 p., $104 \mathrm{pl}$.

TARDy D., I989 - Le décor architectonique de Saintes antique. Les chapiteaux et les bases, Paris, Bordeaux, Aquitania, suppl. 5, $184 \mathrm{p}$.

TARDy D., I994 - Le décor architectonique de Saintes antique, II, Les entablements, Bordeaux, Aquitania, supplément 7, 174 p.

TARDy D., 2005 - Le décor architectonique de Vesunna (Périgueux antique), Aquitania, supplément 12, Bordeaux, 145 p.

TASsignon I., I996 - Iconographie et religion dionysiaques en Gaule Belgique et dans les deux Germanies, Bibliothèque de la Faculté de Philosophie et Lettres de l'Université de Liège, fasc. CCLXV, Liège, 378 p.

Terrer D., Lauxerrois R., Robert R., Gaggadis-Robin V., Hermary A., Jockey Ph. et Lavagne H., 2003 - Nouvel Espérandieu, I, Vienne (Isère), Paris, Académie des inscriptions et belles-lettres, LVII + 254 p. +269 pl.

Triste A. et André P., I 992 - "Fouilles du forum », in AndrÉ P. et Triste A., Quand Vannes s'appelait Darioritum, catalogue d'exposition, Vannes, p. 92-96.

Villers G., I 882 - "Les thermes romains de la ville de Bayeux. Renseignements préliminaires ", Indicateur de Bayeux, n 99, 12 décembre 1882, p. 2.

VIPARD P., I997 - Une domus du quartier des thermes d'Aregenua (Vieux, Calvados). Contribution à l'histoire de l'habitat urbain 
en Gaule romaine, Thèse de doctorat, Paris IV, 1997, 899 p. + 225 fig. + 176 photos + 1 plan.

VIPARD P., I 998 - La Maison du "Bas de Vieux ». Une riche habitation du quartier des thermes d'Aregenua, Caen, éditions du conseil général du Calvados, $125 \mathrm{p}$.

Vipard P., 200 I - "Le rôle du décor dans les parties officielles d'une domus à péristyle du début du $\mathrm{III}^{\mathrm{e}}$ siècle : le cas de la Maison au Grand Péristyle (Vieux, Calvados) », Revue du Nord, 83, no 343, p. 21-33.

WALter H., I975 - La colonne ciselée dans la Gaule romaine, Besançon, Annales littéraires et l'Université de Besançon 119, 140 p. + XXXIX pl.

Walter H. et Bruchet J., I 986 - La Porte Noire de Besançon, Annales littéraires de l'Université de Besançon 321, Paris, Besançon, 2 tomes, 483 p. et 69 fig. + LXXXIX pl.

WAlter H., 2006 - Laissez-vous conter la Porte Noire (catalogue d'exposition), éditions ville de Besançon, Besançon, 27 p.
Wyler S., 2004 - «Dionysos Domesticus. Les motifs dionysiaques dans les maisons pompéiennes et romaines ( $\mathrm{II}^{\mathrm{e}} \mathrm{s}$. av. J.-C.- $\mathrm{I}^{\mathrm{er}} \mathrm{s}$. apr. J.-C.) ", Mélanges de l'École française de Rome, 116, 2, p. 933-951.

Wyler S., 20 I 3 - «An Augustan Trend towards Dionysos: Around the 'Auditorium of Maecenas' ", in Bernabé A., Herrero de Jáuregui M., Jiménez San Cristóbal A.I. et Martín Hernández R. (éd.), Redefining Dionysos, Berlin, New York, Walter de Gruyter, coll. "Mythos Eikon Poiesis; 5 », 2013, p. 541-553.

ZANKeR P., $200 \mathrm{I}$ - Un art pour le plaisir des sens. Le monde figuré de Dionysos et Aphrodite dans l'art hellénistique, Paris, Gérard de Monfort, 103 p.

ZANKer P. et Ewald B.C., 2008 - Vivere con i miti. L'iconografia dei sarcophagi romani, trad. italienne, Turin Bollati Boringhieri (Nuova cultura, 177), 387 p.

Zusammenfassung: Ein monumentaler Bogen aus der Zeit der Severer in Bayeux (Calvados, Frankreich) - Zwanzig Architekturblöcke, die in Bayeux im 19. Jahrhundert entdeckt wurden, sind bis heutige die einzigen Überreste eines monumentalen Bogens aus severischer Zeit. Seine Architektur kann in groben Zügen rekonstruiert werden: das Monument wies drei Tore auf, seine mittlere Öffnung war deutlich grösser als die beiden seitlichen und wies eine reduzierte Mächtigkeit auf, die mehr an eine monumentale Fassade erinnerte als dass sie ein eigenständiges Bauvolumen repräsentierte. Nach den erhaltenen Fragmenten war der Bogen mit umfangreichen Verzierungen versehen, die zwei Themenbereiche behandelten, auf die sich die kaiserliche Macht stützte: der Überfluss und die felicitas.

Resumen: Un arco monumental severiano en Bayeux (Calvados) - Veinte bloques de arquitectura descubiertos en el siglo XIX en Bayeux son los únicos restos conocidos de un arco monumental que data de la época de los Severos. Su arquitectura puede ser restaurada en las grandes líneas: el monumento tenía tres bahias, con una apertura central sustancialmente más grande, y tenía un espesor reducido, que le hacía parecer más a una fachada monumental que a un volumen independiente. Estaba cubierto de una abundante decoración que, a juzgar por los fragmentos conservados, evocaba la abundancia y la felicitas, a las que servía el poder imperial.

Schlüsselwörter: dreitoriger Bogen, Epoche der Severer, Wein, Dionysos.

Palabras clave: arco con tres bahías, época de los Severos, vid, Dionisio. 